\title{
Steenrod squares on intersection cohomology and a conjecture of $M$ Goresky and W Pardon
}

\author{
DAVID CHATAUR \\ MARTINTXO SARALEGI-ARANGUREN \\ DANIEL TANRÉ
}

We prove a conjecture raised by M Goresky and W Pardon, concerning the range of validity of the perverse degree of Steenrod squares in intersection cohomology. This answer turns out to be of importance for the definition of characteristic classes in the framework of intersection cohomology.

For this purpose, we present a construction of cup $_{i}$-products on the cochain complex, built on the blow-up of some singular simplices and introduced in a previous work. We extend to this setting the classical properties of the associated Steenrod squares, including Adem and Cartan relations, for any loose perversities. In the case of a PLpseudomanifold and range $2 \bar{p}$, we prove that our definition coincides with Goresky's definition. We also show that our Steenrod squares are topological invariants which do not depend on the choice of a stratification of $X$.

Several examples of concrete computation of perverse Steenrod squares are given, including the case of isolated singularities, and more especially, we describe the Steenrod squares on the Thom space of a vector bundle as a function of the Steenrod squares of the base space and the Stiefel-Whitney classes of the bundle. We also detail an example of a nontrivial square, $\mathrm{Sq}^{2}: H_{\bar{p}} \rightarrow H_{\bar{p}+2}$, whose information is lost if we consider it as taking values in $H_{2 \bar{p}}$, showing the interest of the Goresky-Pardon conjecture.

$55 \mathrm{~N} 33,55 \mathrm{~S} 10,57 \mathrm{~N} 80$

Intersection cohomology was introduced by M Goresky and R MacPherson in [10] and [11] in order to adapt Poincaré duality to singular manifolds and extend characteristic classes to this paradigm. Steenrod squares on the intersection cohomology of a pseudomanifold $X$ were already defined and studied by Goresky in [13]. For that, he uses a sheaf introduced by Deligne and proves that the Steenrod construction of cup $_{i}$-products induces a morphism $\mathrm{Sq}_{G}^{i}: H_{\bar{p}}^{r}\left(X ; \mathbb{F}_{2}\right) \rightarrow H_{2}^{r}{ }^{+i}\left(X ; \mathbb{F}_{2}\right)$ for any Goresky-MacPherson perversity $\bar{p}$ such that $2 \bar{p}(\ell) \leq \ell-2$ for any $\ell$, where $\mathbb{F}_{2}$ is the field with two elements.

Here we consider the blow-up $\tilde{N}^{*}(X)$ of the normalized cochain complex on a filtered version of the singular simplicial set associated to $X$. This notion of blow-up, which we 
defined in [4] and recall in Section 1, comes from a version adapted to differential forms already existent in Brasselet, Hector, and Saralegi [3]. The elements of $\tilde{N}^{*}(X)$ have a perverse degree (see Definition 1.2) which allows the definition of a complex $\tilde{N}_{\bar{p}}^{*}(X)$ for any loose perversity $\bar{p}$. In [4], we have proved that the blow-up $\widetilde{C}^{*}(X)$ gives the Goresky-MacPherson intersection cohomology of the pseudomanifold $X$ for the complementary perversity when we are working over a field. With Proposition 1.5 , the blow-up $\tilde{N}^{*}(X)$ inherits this property; we denote its cohomology by $H_{\mathrm{TW}, *}^{\bullet}\left(X ; \mathbb{F}_{2}\right)$.

When the coefficients of $\tilde{N}^{*}(X)$ are in $\mathbb{F}_{2}$, we define a structure of cup -products $\cup_{i}: \tilde{N}_{\bar{p}}^{*}(X) \otimes \tilde{N}_{\bar{q}}^{*}(X) \rightarrow \tilde{N}_{\bar{p}+\bar{q}}^{*}(X)$ for any loose perversities $\bar{p}$ and $\bar{q}$. This is done following the work of C Berger and B Fresse in [1] (see also May [18]): we consider a normalized, homogeneous bar resolution $\mathcal{E}(2)$ of the symmetric group $\Sigma_{2}$ and prove that there exists a $\Sigma_{2}$-equivariant cochain map

$$
\psi_{2}: \mathcal{E}(2) \otimes \tilde{N}_{\bar{p}}^{*}(X) \otimes \tilde{N}_{\bar{q}}^{*}(X) \rightarrow \tilde{N}_{\bar{p}+\bar{q}}^{*}(X) .
$$

Such a map is called a structure of a perverse $\mathcal{E}(2)$-algebra on $\tilde{N}_{\bullet}^{*}(X)$; its construction comes from the existence of a diagonal on $\mathcal{E}(2)$, established in [1]. Moreover, we prove in Theorem A that the $\operatorname{cup}_{i}$-products arising from the existence of $\psi_{2}$ verify the two properties $a \cup_{|a|} a=a$ and $a \cup_{i} a^{\prime}=0$ if $i \geq \min \left(|a|,\left|a^{\prime}\right|\right)$, where $|a|,\left|a^{\prime}\right|$ are the respective degrees of $a$ and $a^{\prime}$.

The definition of perverse $\mathcal{E}(2)$-algebras can be extended to perverse $\mathcal{E}(n)$-algebras for any $n$. As this work is concerned with Steenrod squares, we consider only perverse $\mathcal{E}(2)$-algebras over $\mathbb{F}_{2}$. Nevertheless, it is clear that our methods of proof can be enhanced to give a structure of perverse $E_{\infty}$-algebras over $\mathbb{Z}$ on $\tilde{N}_{\bullet}^{*}(X)$. We will come back on these points in a forthcoming paper.

As usual, Steenrod squares are defined on $H_{\mathrm{TW}, \bar{p}}^{k}\left(X ; \mathbb{F}_{2}\right)$ by $\operatorname{Sq}^{i}(a)=a \cup_{k-i} a$. Using May's presentation of Steenrod squares in [18], we see that the classical properties of Steenrod squares are direct consequences of the structure of perverse $\mathcal{E}(2)$-algebras. We collect them, together with Adem and Cartan relations, in Theorem B. (One may observe that the proof of the Adem relation on a tensor product needs a brief incursion in the world of perverse $\mathcal{E}(4)$-algebras over $\mathbb{F}_{2}$.)

In Theorem B, we also answer positively to the problem asked by Goresky in [13, page 493] and to the conjecture made by Goresky and Pardon in [12, Conjecture 7.5]. This problem concerns the range of the perversities: with the definition of Steenrod squares via the cup ${ }_{i}$-products, it is clear that $\operatorname{Sq}^{i}$ sends $H_{\mathrm{TW}, \bar{p}}^{k}\left(X ; \mathbb{F}_{2}\right)$ into $H_{\mathrm{TW}, 2 \bar{p}}^{k+i}\left(X ; \mathbb{F}_{2}\right)$. We prove that, in fact, there is a lifting as a map,

$$
\mathrm{Sq}^{i}: H_{\mathrm{TW}, \bar{p}}^{k}\left(X ; \mathbb{F}_{2}\right) \rightarrow H_{\mathrm{TW}, \mathcal{L}(\bar{p}, i)}^{k+i}\left(X ; \mathbb{F}_{2}\right),
$$


where $\mathcal{L}(\bar{p}, i)$ is the loose perversity defined by $\mathcal{L}(\bar{p}, i)(\ell)=\min (2 \bar{p}(\ell), \bar{p}(\ell)+i)$, which is exactly [12, Conjecture 7.5]. This reveals an important fact because it allows the lifting of $\mathrm{Wu}$ classes in intersection cohomology, in a lower part of the poset of perversities.

In Theorem C, we prove that our definition of Steenrod squares coincides with Goresky's definition introduced in [13]. For doing that, we transform the blow-up $\tilde{N}_{\bullet}^{*}$ into a sheaf $\mathbf{I N} *$ on $X$ and prove that $\mathbf{I N}_{\bullet}^{*}$ is isomorphic to the Deligne sheaf in the derived category of sheaves on $X$. The rest of the proof comes from a unicity theorem for Steenrod squares defined on an injective sheaf, established by Goresky [13].

We end this part of the work with examples of concrete computation of perverse Steenrod squares, beginning with the case of isolated singularities. From it, we are able to write the Steenrod squares on the intersection cohomology of the Thom space associated to a vector bundle as a function of the Steenrod squares of the base space and the Stiefel-Whitney classes of the bundle. We also detail an example of a nontrivial square $\mathrm{Sq}^{2}: H_{\mathrm{TW}, \bar{p}}\left(X ; \mathbb{F}_{2}\right) \rightarrow H_{\mathrm{TW}, \mathcal{L}(\bar{p}, 2)}\left(X ; \mathbb{F}_{2}\right)$ whose information is lost if we consider it as values in $H_{\mathrm{TW}, 2 \bar{p}}$, showing the interest of the Goresky-Pardon conjecture. This last example can also be seen as a tubular neighborhood of a stratum, which is the first step in the study of intersection cohomology of pseudomanifolds.

In Theorem D, we prove that Steenrod squares, $\mathrm{Sq}^{i}: H_{\mathrm{TW}, \bar{p}}^{r}\left(X ; \mathbb{F}_{2}\right) \rightarrow H_{\mathrm{TW}, \mathcal{L}(\bar{p}, i)}^{r+i}\left(X ; \mathbb{F}_{2}\right)$, are topological invariants when $X$ is a PL-pseudomanifold. This completes the result of [13] that the Steenrod squares are topological invariants as homomorphisms $H \bar{p}\left(X ; \mathbb{F}_{2}\right) \rightarrow H_{2}^{r} \bar{p} i\left(X ; \mathbb{F}_{2}\right)$. The proof is combinatorial, using the description of Steenrod squares made by Steenrod in [22].

We now emphasize some particularities which are important in the process of the proof of the Goresky-Pardon conjecture. The main point is that our technique allows an explicit construction of the cup $_{i}$-products at the level of cochain complexes without requiring the derived category for their definition. In the context of filtered objects, observe first that the notion of filtered singular simplices is a natural one; see Remark 1.7.

The second modus operandi is the blow-up of these simplices. In differential geometry, a blow-up is the replacement of a submanifold $N$ of a manifold $M$ by the boundary of a tubular neighborhood of $N$ in $M$. Its simplicial version can be illustrated as follows in the case of $\Delta=\Delta^{j_{0}} * \Delta^{j_{1}}$ : we cut off a small open neighborhood of $\Delta^{j_{0}}$ in $\Delta$ to get $\widetilde{\Delta}=c \Delta^{j_{0}} \times \Delta^{j_{1}}$; see Figure 1 .

In the general case of $\Delta=\Delta^{j_{0}} * \cdots * \Delta^{j_{n}}$, we use an inductive process which consists in cutting off a small open neighborhood of the smallest stratum; see Figure 2.

The faces containing $\Delta^{j_{i}} \times\{1\}$ as a factor, which play a fundamental role in the definition of the perverse degree (see Definition 1.2), have been shadowed in these figures. 


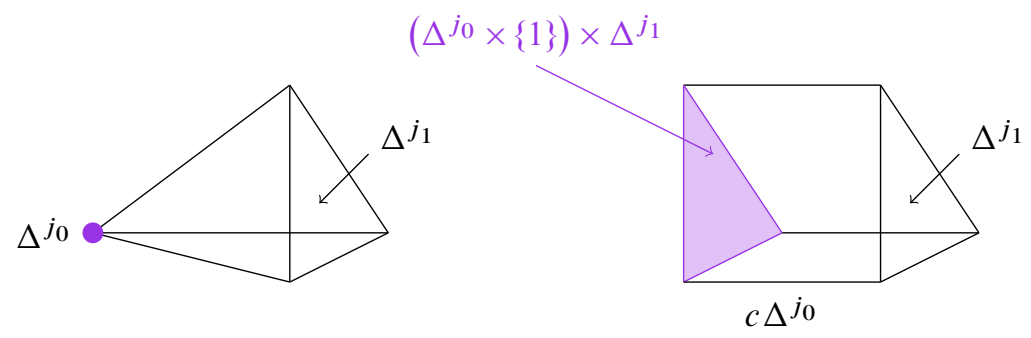

Figure 1: $\Delta=\Delta^{j_{0}} * \Delta^{j_{1}}$ (left) has for blow-up $\widetilde{\Delta}=c \Delta^{j_{0}} \times \Delta^{j_{1}}$ (right).

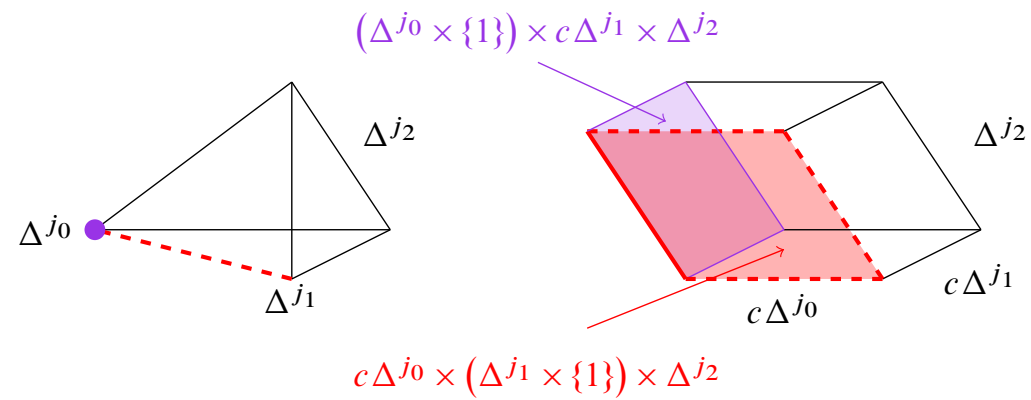

Figure 2: $\Delta=\Delta^{j_{0}} * \Delta^{j_{1}} * \Delta^{j_{2}}$ (left) has for blow-up $\widetilde{\Delta}=c \Delta^{j_{0}} \times c \Delta^{j_{1}} \times \Delta^{j_{2}}$ (right).

The motivation for such process occurs when one determines the intersection cohomology of a pseudomanifold with differential forms: as these forms cannot be defined on the singular strata, the only possibility is to define them on the regular part and ask for some control in the neighborhood of strata. That is what we do here for cochains. As observed in [8] by G Friedman and J E McClure, the classical way for the definition of a cup-product (with back and front faces) does not fit with perverse degrees. But one advantage of the blow-up is that we can define the cup-product (and more generally, the $\mathrm{cup}_{i}$-products) stratum after stratum, on each factor of the product $c \Delta^{j_{0}} \times \cdots \times c \Delta^{j_{n-1}} \times \Delta^{j_{n}}$, from the classical definition and in a compatible way with the perverse structure. Finally, this procedure reveals itself of an easy use and does not lose any information in cohomology; it gives the same structure on cohomology as Goresky's definition as it is established in Section 4.

In Section 1, we recall basic notions concerning filtered face sets and their intersection cohomology. Section 2 is devoted to the construction of a structure of a perverse $\mathcal{E}(2)-$

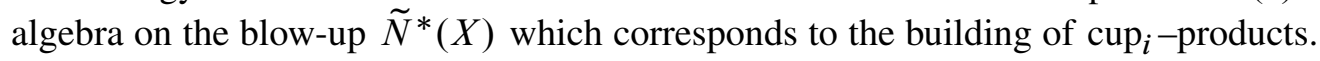
In Section 3, we establish the main properties of perverse Steenrod squares, including the proof of the perverse range conjecture of Goresky and Pardon. The comparison between our definition and Goresky's definition of Steenrod squares in the case of a 
pseudomanifold is done in Section 4. The particular case of isolated singularities and the treatment of Steenrod squares in the intersection cohomology of a Thom space are presented in Section 5. An example of a square $\mathrm{Sq}^{2}$ in the intersection cohomology of the total space of a fibration whose fiber is a cone is given in Section 6. This example shows the interest of having a range of perversity in $\mathcal{L}(\bar{p}, i)$ instead of $2 \bar{p}$. Finally, Section 7 is devoted to the topological invariance of our Steenrod squares.

All the cohomology groups appearing in this text are over the field with two elements, $\mathbb{F}_{2}$. If there is no ambiguity, we simplify the notation $H^{*}\left(X ; \mathbb{F}_{2}\right)$ in $H^{*}(X)$.

Acknowledgements We thank the anonymous referee for her/his comments and suggestions which have contributed to improve the organization and the writing. The third author is partially supported by the MICINN grant MTM2013-41768-P, ANR-11-BS01002-01 "HOGT" and ANR-11-LABX-0007-01 "CEMPI"

\section{Blow-up and perversity}

In this section, we recall the basics of a simplicial version of intersection cohomology, already introduced in [4].

Let $\Delta^{k}$ be the standard simplex of $\mathbb{R}^{k+1}$, whose vertices $v_{0}, \ldots, v_{k}$ verify $v_{i}=$ $\left(t_{0}, \ldots, t_{k}\right)$ with $t_{j}=0$ if $j \neq i$ and $t_{i}=1$. Let $\delta_{i}:\{0,1, \ldots, k-1\} \rightarrow\{0,1, \ldots, k\}$ be defined by

$$
\delta_{i}(j)= \begin{cases}j & \text { if } j<i \\ j+1 & \text { if } j \geq i .\end{cases}
$$

Such maps generate linear applications, still denoted $\delta_{i}: \Delta^{k-1} \rightarrow \Delta^{k}$ and defined by $\delta_{i}\left(v_{j}\right)=v_{\delta_{i}(j)}$. More generally, any map $\sigma:\{0,1, \ldots, \ell\} \rightarrow\{0,1, \ldots, k\}$ generates a linear application $\sigma: \Delta^{\ell} \rightarrow \Delta^{k}$.

We fix an integer $n$ and consider the category $\Delta_{\mathcal{F}}^{[n]}$ whose

- objects are the joins $\Delta=\Delta^{j_{0}} * \Delta^{j_{1}} * \cdots * \Delta^{j_{n}}$, where $\Delta^{j_{i}}$ is the simplex of dimension $j_{i}$, possibly empty, with the conventions $\Delta^{-1}=\varnothing$ and $\varnothing * X=X$;

- maps are the $\sigma: \Delta=\Delta^{j_{0}} * \Delta^{j_{1}} * \cdots * \Delta^{j_{n}} \rightarrow \Delta^{\prime}=\Delta^{k_{0}} * \Delta^{k_{1}} * \cdots * \Delta^{k_{n}}$, of the shape $\sigma=*_{i=0}^{n} \sigma_{i}$, with $\sigma_{i}:\left\{0,1, \ldots, j_{i}\right\} \rightarrow\left\{0,1, \ldots, k_{i}\right\}$ an injective order-preserving map for each $i$.

The category $\Delta_{\mathcal{F}}^{[n],+}$ is the full subcategory of $\Delta_{\mathcal{F}}^{[n]}$ whose objects are the joins $\Delta^{j_{0}} * \Delta^{j_{1}} * \cdots * \Delta^{j_{n}}$ with $\Delta^{j_{n}} \neq \varnothing$; ie $j_{n} \geq 0$. To any such element, we associate its blow-up which is the map

$$
\mu: \tilde{\Delta}=c \Delta^{j_{0}} \times \cdots \times c \Delta^{j_{n-1}} \times \Delta^{j_{n}} \rightarrow \Delta=\Delta^{j_{0}} * \cdots * \Delta^{j_{n}}
$$


defined by

$$
\begin{aligned}
\mu\left(\left[y_{0}, s_{0}\right], \ldots,\left[y_{n-1}, s_{n-1}\right], y_{n}\right) & \\
=s_{0} y_{0}+\left(1-s_{0}\right) s_{1} y_{1}+\cdots+(1- & \left.s_{0}\right) \cdots\left(1-s_{n-2}\right) s_{n-1} y_{n-1} \\
& +\left(1-s_{0}\right) \cdots\left(1-s_{n-2}\right)\left(1-s_{n-1}\right) y_{n}
\end{aligned}
$$

where $y_{i} \in \Delta^{j_{i}}$ and $\left[y_{i}, s_{i}\right] \in c \Delta^{j_{i}}=\left(\Delta^{j_{i}} \times[0,1]\right) /\left(\Delta^{j_{i}} \times\{0\}\right)$. The prism $\tilde{\Delta}$ is sometimes also called the blow-up of $\Delta$.

Observe that this blow-up is well defined thanks to the restriction to the subcategory $\Delta_{\mathcal{F}}^{[n],+}$. In the topological setting (see Remark 1.7), this restriction means that we do not consider simplices entirely included in the singular part.

Definition 1.1 A filtered face set, of formal dimension $n$, is a contravariant functor $\underline{K}$

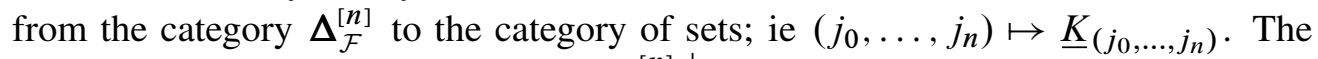
restriction of the filtered face set $\underline{K}$ to $\Delta_{\mathcal{F}}^{[n],+}$ is denoted $\underline{K}_{+}$.

If $\underline{K}$ and $\underline{K}^{\prime}$ are filtered face sets, a filtered face map $f: \underline{K} \rightarrow \underline{K}^{\prime}$ is a natural transformation between the two functors $\underline{K}$ and $\underline{K}^{\prime}$. We denote by $\Delta_{\mathcal{F}}^{[n]}$-Sets the category of filtered face sets.

To any simplicial set $Y$, we can associate the $\mathbb{F}_{2}$-vector space $C_{d}(Y)$ generated by the $d$-dimensional simplices of $Y$. The normalized chain complex $N_{d}(Y)$ is the quotient of $C_{d}(Y)$ by the degeneracies $\mathfrak{s}_{i}$ :

$$
N_{d}(Y)=C_{d}(Y) / \mathfrak{s}_{0} C_{d-1}(Y)+\cdots+\mathfrak{s}_{d-1} C_{d-1}(Y) .
$$

We consider also the duals

$$
N^{*}(Y)=\operatorname{hom}_{\mathbb{F}_{2}}\left(N_{*}(Y), \mathbb{F}_{2}\right) \quad \text { and } \quad C^{*}(Y)=\operatorname{hom}_{\mathbb{F}_{2}}\left(C_{*}(Y), \mathbb{F}_{2}\right) .
$$

Any face operator, $\delta_{i}: \Delta^{j_{\ell}} \rightarrow \Delta^{j_{\ell}+1}$ for some $\ell \in\{0, \ldots, n-1\}$, induces a chain map $\delta_{i}^{*}: N^{*}\left(c \Delta^{j_{0}}\right) \otimes \cdots \otimes N^{*}\left(c \Delta^{j_{\ell}+1}\right) \otimes \cdots \otimes N^{*}\left(\Delta^{j_{n}}\right)$

$$
\rightarrow N^{*}\left(c \Delta^{j_{0}}\right) \otimes \cdots \otimes N^{*}\left(c \Delta^{j_{\ell}}\right) \otimes \cdots \otimes N^{*}\left(\Delta^{j_{n}}\right)
$$

defined by the identity on the factors in $\Delta^{j_{i}}$ for $i \neq \ell$.

We denote also by $\delta_{i}: \Delta^{j_{0}} * \cdots * \Delta^{j_{\ell}} * \cdots * \Delta^{j_{n}} \rightarrow \Delta^{j_{0}} * \cdots * \Delta^{j_{\ell}+1} * \cdots * \Delta^{j_{n}}$ the operator defined by $\delta_{i}: \Delta^{j_{\ell}} \rightarrow \Delta^{j_{\ell}+1}$ and the identity maps. Additionally, for any simplex $\sigma: \Delta^{j_{0}} * \cdots * \Delta^{j_{\ell}+1} * \cdots * \Delta^{j_{n}} \rightarrow \underline{K}_{+}$, we define by $\partial_{i} \sigma=\sigma \circ \delta_{i}$ a simplex $\partial_{i} \sigma: \Delta^{j_{0}} * \cdots * \Delta^{j_{\ell}} * \cdots * \Delta^{j_{n}} \rightarrow \underline{K}$, and a complex

$$
\tilde{N}_{\sigma}^{*}=N^{*}\left(c \Delta^{j_{0}}\right) \otimes \cdots \otimes N^{*}\left(c \Delta^{j_{n-1}}\right) \otimes N^{*}\left(\Delta^{j_{n}}\right) .
$$


These previous considerations on face operators can easily be adapted to the case $\ell=n$.

A global section (or cochain) on $\underline{K}$ is a function which assigns to each simplex $\sigma \in \underline{K}_{+}$ an element $c_{\sigma} \in \tilde{N}_{\sigma}^{*}$ such that $c_{\partial_{i} \sigma}=\delta_{i}^{*}\left(c_{\sigma}\right)$ for all $\sigma \in \underline{K}+$ and all $\delta_{i} \in \Delta_{\mathcal{F}}^{[\overline{n]}},+$. (The restriction to $\Delta_{\mathcal{F}}^{[n],+}$ implies $\Delta^{j_{n}} \neq \varnothing$.)

The space of global sections is denoted by $\tilde{N}^{*}(\underline{K})$ and called the blow-up of $N^{*}$ over the filtered face set $\underline{K}$. Global sections have an extra degree, called the perverse degree, that we describe now.

Let $\sigma: \Delta^{j_{0}} * \cdots * \Delta^{j_{n}} \rightarrow \underline{K}_{+}$and $\ell \in\{1, \ldots, n\}$ such that $\Delta^{j_{n-\ell}} \neq \varnothing$. For any cochain $c_{\sigma} \in N^{*}\left(c \Delta^{j_{0}}\right) \otimes \cdots \otimes N^{*}\left(c \Delta^{j_{n-1}}\right) \otimes N^{*}\left(\Delta^{j_{n}}\right)$, its restriction

(1) $c_{\sigma, n-\ell} \in N^{*}\left(c \Delta^{j_{0}}\right) \otimes \cdots \otimes N^{*}\left(\Delta^{j_{n-\ell}} \times\{1\}\right) \otimes \cdots \otimes N^{*}\left(c \Delta^{j_{n-1}}\right) \otimes N^{*}\left(\Delta^{j_{n}}\right)$

can be written $c_{\sigma, n-\ell}=\sum_{k} c_{\sigma, n-\ell}^{\prime}(k) \otimes c_{\sigma, n-\ell}^{\prime \prime}(k)$, with

- $c_{\sigma, n-\ell}^{\prime}(k) \in N^{*}\left(c \Delta^{j_{0}}\right) \otimes \cdots \otimes N^{*}\left(c \Delta^{j_{n-\ell-1}}\right) \otimes N^{*}\left(\Delta^{j_{n-\ell}} \times\{1\}\right)$ and

- $c_{\sigma, n-\ell}^{\prime \prime}(k) \in N^{*}\left(c \Delta^{j_{n-\ell+1}}\right) \otimes \cdots \otimes N^{*}\left(\Delta^{j_{n}}\right)$.

Observe that each term of the tensor product in formula (1) has a finite canonical basis, and the decomposition of $c_{\sigma, n-\ell}$ can be canonically chosen as a function of the associated basis of the tensor product.

Definition 1.2 If $c_{\sigma, n-\ell} \neq 0$, the $\ell$-perverse degree $\left\|c_{\sigma}\right\|_{\ell}$ of $c_{\sigma}$ is equal to

$$
\left\|c_{\sigma}\right\|_{\ell}=\sup _{k}\left\{\left|c_{\sigma, n-\ell}^{\prime \prime}(k)\right| \text { such that } c_{\sigma, n-\ell}^{\prime}(k) \neq 0\right\},
$$

where $\left|c_{\sigma, n-\ell}^{\prime \prime}(k)\right|$ denotes the usual degree of the cochain $c_{\sigma, n-\ell}^{\prime \prime}(k)$. If $c_{\sigma, n-\ell}=0$ or $\Delta^{j_{n-\ell}}=\varnothing$, we set $\left\|c_{\sigma}\right\|_{\ell}=-\infty$.

The perverse degree of a global section $c \in \tilde{N}^{*}(\underline{K})$ is the $n$-tuple

$$
\|c\|=\left(\|c\|_{1}, \ldots,\|c\|_{n}\right),
$$

where $\|c\|_{\ell}$ is the supremum of the $\left\|c_{\sigma}\right\|_{\ell}$ for all $\sigma \in \underline{K}_{+}$.

Intersection cohomology requires a notion of perversity that we now introduce, following the convention of [15].

Definition 1.3 A loose perversity is a map $\bar{p}: \mathbb{N} \rightarrow \mathbb{Z}, i \mapsto \bar{p}(i)$ such that $\bar{p}(0)=0$. A perversity is a loose perversity such that $\bar{p}(i) \leq \bar{p}(i+1) \leq \bar{p}(i)+1$ for all $i \in \mathbb{N}$. A Goresky-MacPherson perversity (or GM-perversity) is a perversity such that $\bar{p}(1)=$ $\bar{p}(2)=0$. 
If $\bar{p}_{1}$ and $\bar{p}_{2}$ are two loose perversities, we set $\bar{p}_{1} \leq \bar{p}_{2}$ if we have $\bar{p}_{1}(i) \leq \bar{p}_{2}(i)$ for all $i \in \mathbb{N}$. The poset of all loose perversities is denoted $\mathcal{P}_{\text {loose }}^{n}$.

The lattice of GM-perversities, denoted $\mathcal{P}^{n}$, admits a maximal element $\bar{t}$ called the top perversity, defined by $\bar{t}(i)=i-2$ if $i \geq 2$, and $\bar{t}(0)=\bar{t}(1)=0$.

To these posets, we add an element $\bar{\infty}$ which is the constant map to $\infty$. We call it the infinite perversity despite the fact that it is not a perversity in the sense of the previous definition. Finally, we set $\hat{\mathcal{P}}^{n}=\mathcal{P}^{n} \cup\{\bar{\infty}\}$ and $\widehat{\mathcal{P}}_{\text {loose }}^{n}=\mathcal{P}_{\text {loose }}^{n} \cup\{\bar{\infty}\}$.

Definition 1.4 Let $\bar{p}$ be a loose perversity. A global section $c \in \tilde{N}^{*}(\underline{K})$ is called $\bar{p}$-admissible if $\|c\|_{i} \leq \bar{p}(i)$ for any $i \in\{1, \ldots, n\}$, and a global section $c$ is of $\bar{p}$-intersection if $c$ and its differential $\delta c$ are $\bar{p}$-admissible.

We denote by $\tilde{N}_{\bar{p}}^{*}(\underline{K})$ the complex of global sections of $\bar{p}$-intersection and by $H_{\bar{p}}^{*}(\underline{K} ; \tilde{N})$ its homology.

By using the same process with $C^{*}$ in place of $N^{*}$, we obtain a second complex of global sections of $\bar{p}$-intersection $\widetilde{C}_{\bar{p}}^{*}(\underline{K})$ of homology $H_{\bar{p}}^{*}(\underline{K} ; \widetilde{C})$. Directly from [4, Theorem A], we get an isomorphism between these two cohomologies.

Proposition 1.5 Let $\underline{K}$ be a filtered face set and $\bar{p}$ a loose perversity. The canonical surjection $C_{*}(-) \rightarrow \bar{N}_{*}(-)$ induces a quasi-isomorphism $\tilde{N}_{\bar{p}}^{*}(\underline{K}) \rightarrow \tilde{C}_{\bar{p}}^{*}(\underline{K})$ and, therefore, an isomorphism $H_{\bar{p}}^{*}(\underline{K} ; \tilde{N}) \cong H_{\bar{p}}^{*}(\underline{K} ; \widetilde{C})$.

If there is no ambiguity, we denote by $H_{\mathrm{TW}, \bar{p}}^{*}(\underline{K})$ this common value and called it the Thom-Whitney cohomology (henceforth TW-cohomology) of $\underline{K}$ with coefficients in $\mathbb{F}_{2}$ for the loose perversity $\bar{p}$.

The topological objects corresponding to the filtered face sets are locally conical, stratified topological spaces. Here we only consider the case of pseudomanifolds defined as follows.

Definition 1.6 An $n$-dimensional topological pseudomanifold is a nonempty topological space with a filtration by closed subsets

$$
\varnothing=X_{-1} \subseteq X_{0} \subseteq \cdots \subseteq X_{n-2}=X_{n-1} \varsubsetneqq X_{n}=X
$$

such that $X_{i} \backslash X_{i-1}$ is an $i$-dimensional metrizable topological manifold, or the empty set, for all $i$. Moreover, for each point $x \in X_{i} \backslash X_{i-1}$ with $i \neq n$, there exist: 
(a) an open neighborhood $V$ of $x$ in $X$, endowed with the induced filtration;

(b) an open neighborhood $U$ of $x$ in $X_{i} \backslash X_{i-1}$;

(c) a compact topological pseudomanifold $L=\left(L_{j}\right)_{0 \leq j \leq n-i-1}$ with dimension $n-i-1$, whose cone $\stackrel{\circ}{c} L=(L \times[0,1[) /(L \times\{0\})$ is endowed with the conic filtration, ie $(\stackrel{\circ}{c} L)_{i}=\stackrel{\circ}{c} L_{i-1}$ for $i \geq 0$;

(d) a homeomorphism $\varphi: U \times \stackrel{\circ}{c} L \rightarrow V$ such that

(1) $\varphi(u, \mathrm{v})=u$ for any $u \in U$, with $\mathrm{v}$ the cone point,

(2) $\varphi\left(U \times \stackrel{\circ}{c} L_{j}\right)=V \cap X_{i+j+1}$ for any $j \in\{0, \ldots, n-i-1\}$.

The couple $(V, \varphi)$ is called a conic chart of $x$ and the filtered space $L$ the link of $x$.

This definition makes sense with an induction on the dimension, starting from pseudomanifolds of dimension 0 , which are discrete topological spaces by definition. Also, one can prove that the subspace $X_{n} \backslash X_{n-2}$ is dense.

Remark 1.7 The set of filtered singular simplices is the bridge between pseudomanifolds and the more general notion of filtered face sets. More precisely, for any pseudomanifold $X$ we define (see [4, Example 1.5]) the singular filtered face set by

$$
\underline{\operatorname{ISing}}^{\mathcal{F}}(X)_{j_{0}, \ldots, j_{n}}=\left\{\sigma: \Delta^{j_{0}} * \cdots * \Delta^{j_{n}} \rightarrow X \mid \sigma^{-1} X_{i}=\Delta^{j_{0}} * \cdots * \Delta^{j_{i}}\right\} .
$$

Such simplex is called filtered.

If $X$ is a pseudomanifold and $\underline{K}=\underline{\operatorname{ISing}}^{\mathcal{F}}(X)$, we use the notations $\tilde{N}_{\bar{p}}^{*}(X), \widetilde{C}_{\bar{p}}^{*}(X)$ and $H_{\mathrm{TW}, \bar{p}}^{*}(X)$ for the Thom-Whitney complexes and their cohomology. (As $X_{n-1}=$ $X_{n-2}$, the case $i=1$ in Definition 1.4 is vacuous in this setting.)

We end this section with a reminder of Goresky-MacPherson cohomology (with coefficients in $\mathbb{F}_{2}$ ) and its link with the blow-up. Let $\bar{p}$ be a loose perversity. A filtered simplex $\sigma: \Delta=\Delta^{j_{0}} * \cdots * \Delta^{j_{n}} \rightarrow X$ has a perverse degree $\|\sigma\|=\left(\|\sigma\|_{0}, \ldots,\|\sigma\|_{n}\right)$, where $\|\sigma\|_{\ell}=\operatorname{dim}\left(\Delta^{j_{0}} * \cdots * \Delta^{j_{n-\ell}}\right)$, with $\|\sigma\|_{\ell}=-\infty$ if $\sigma^{-1} X_{n-\ell}=\varnothing$.

A $\bar{p}$-admissible simplex of $X$ is a filtered simplex $\sigma: \Delta=\Delta^{j_{0}} * \cdots * \Delta^{j_{n}} \rightarrow X$ such that $\|\sigma\|_{\ell} \leq \operatorname{dim} \Delta-\ell+\bar{p}(\ell)$ for any $\ell \in\{1, \ldots, n\}$. A $\bar{p}$-admissible chain is a linear combination of $\bar{p}$-admissible simplices. A chain $c$ is of $\bar{p}$-intersection if $c$ and its boundary $\partial c$ are $\bar{p}$-admissible. Denote by $C_{*}^{\mathrm{GM}, \bar{p}}(X)$ and $N_{*}^{\mathrm{GM}, \bar{p}}(X)$ the complexes of $\bar{p}$-intersection chains, by $C_{\mathrm{GM}, \bar{p}}^{*}(X)=\operatorname{hom}\left(C_{*}^{\mathrm{GM}, \bar{p}}(X), \mathbb{F}_{2}\right)$ and $N_{\mathrm{GM}, \bar{p}}^{*}(X)=\operatorname{hom}\left(N_{*}^{\mathrm{GM}, \bar{p}}(X), \mathbb{F}_{2}\right)$ their duals, and by $H_{\mathrm{GM}, \bar{p}}^{*}(X)=H\left(C_{\mathrm{GM}, \bar{p}}^{*}(X)\right)=$ $H\left(N_{\mathrm{GM}, \bar{p}}^{*}(X)\right)$ their homology, called the Goresky-MacPherson [10] intersection 
cohomology of $X$ (henceforth GM-cohomology) with coefficients in $\mathbb{F}_{2}$. This cohomology is isomorphic to the original Goresky-MacPherson cohomology in the case of a pseudomanifold $X$ and a GM-perversity $\bar{p}$ see [4, Proposition A.29] and [15].

The GM and TW cohomologies are related in [4, Theorem B] that we recall here.

Proposition 1.8 Let $X$ be a pseudomanifold, and let $\bar{p}$ and $\bar{q}$ be two perversities such that $\bar{q} \geq 0$ and $\bar{p}(i)+\bar{q}(i)=i-2$ for any $i \in\{2, \ldots, n\}$. Then there is an isomorphism between the GM and the TW cohomologies: $H_{\mathrm{TW}, \bar{q}}^{*}(X) \cong H_{\mathrm{GM}, \bar{p}}^{*}(X)$.

\section{Perverse $\mathcal{E}(2)$-algebras and filtered face sets}

Steenrod squares are built from an action of a normalized homogeneous bar resolution $\mathcal{E}(2)$ of the symmetric group $\Sigma_{2}$ on the normalized singular cochains. This is the way the noncommutativity of the cup-product is controlled up to higher coherent homotopies. This action enriches the multiplicative structure given by the cup-product. We first review it in order to adapt this construction to the perverse setting.

Recall that the resolution $\mathcal{E}(2)$ of $\mathbb{F}_{2}$ as a $\Sigma_{2}$-module is defined by

$$
\cdots \rightarrow \mathcal{E}(2)_{i} \stackrel{d}{\longrightarrow} \mathcal{E}(2)_{i-1} \rightarrow \cdots
$$

with $\mathcal{E}(2)_{i}=\mathbb{F}_{2}\left(e_{i}, \tau_{i}\right)$ and $d e_{i}=d \tau_{i}=e_{i-1}+\tau_{i-1}$. (As we are using cochain complexes, $\mathcal{E}(2)$ is negatively graded.) From the isomorphism $\Sigma_{2} \cong\left\{e_{i}, \tau_{i}\right\}$ with $\tau_{i}$ the generator of $\Sigma_{2}$, the (left) action of $\Sigma_{2}$ defines a natural action on $\mathcal{E}(2)$. This action is extended to the tensor product $\mathcal{E}(2) \otimes \mathcal{E}(2)$ as a diagonal action. Moreover, the complex $\mathcal{E}(2)$ is equipped with a $\Sigma_{2}$-equivariant diagonal $\mathcal{D}: \mathcal{E}(2) \rightarrow \mathcal{E}(2) \otimes \mathcal{E}(2)$ defined by

$$
\mathcal{D}\left(e_{i}\right)=\sum_{j=0}^{i} e_{j} \otimes \tau^{j} . e_{i-j},
$$

with $\tau . e_{k}=\tau_{k}$ and $\tau . \tau_{k}=e_{k}$. This diagonal is essential for the definition of the structure of the $\mathcal{E}(2)$-algebra on $\tilde{N}^{*}(\underline{K})$. Finally, observe that, for any vector space $V$, there is a $\Sigma_{2}$-action on $\operatorname{hom}_{\mathbb{F}_{2}}\left(V^{\otimes 2}, V\right)$ defined by $(\tau . f)\left(v_{1} \otimes v_{2}\right)=f\left(v_{2} \otimes v_{1}\right)$.

Definition 2.1 An $\mathcal{E}(2)$-algebra structure on a cochain complex $A^{*}$ is a cochain map $\psi: \mathcal{E}(2) \otimes A^{\otimes 2} \rightarrow A$ which is $\Sigma_{2}$-equivariant as map from $\mathcal{E}(2)$ to $\operatorname{hom}_{\mathbb{F}_{2}}\left(A^{\otimes 2}, A\right)$.

If we denote $\psi\left(e_{i} \otimes x_{1} \otimes x_{2}\right)$ by $x_{1} \cup_{i} x_{2}$, the previous definition is equivalent to

(1) $\psi\left(\tau_{i} \otimes x_{1} \otimes x_{2}\right)=\psi\left(e_{i} \otimes x_{2} \otimes x_{1}\right)=x_{2} \cup_{i} x_{1}$, together with

(2) the Leibniz condition

$$
\delta\left(x_{1} \cup_{i} x_{2}\right)=x_{1} \cup_{i-1} x_{2}+x_{2} \cup_{i-1} x_{1}+\delta x_{1} \cup_{i} x_{2}+x_{1} \cup_{i} \delta x_{2} .
$$


This means that an $\mathcal{E}(2)$-algebra structure is given by a cochain map, called a cup $_{i}-$ product $\cup_{i}: A^{r} \otimes A^{s} \rightarrow A^{r+s-i}$, satisfying the previous Leibniz condition.

Let $L$ be a simplicial set. In [1], C Berger and B Fresse prove the existence of an $\mathcal{E}(2)$-action on the normalized cochain complex of $L$, ie the existence of a cochain map

$$
\psi_{L}: \mathcal{E}(2) \otimes N^{*}(L)^{\otimes 2} \rightarrow N^{*}(L), \quad e_{i} \otimes x_{1} \otimes x_{2} \mapsto x_{1} \cup_{i} x_{2}
$$

which satisfies the requirements of Definition 2.1. As established by May in [18], classical properties of $\operatorname{cup}_{i}$-products are a direct consequence of this $\mathcal{E}(2)$-algebra structure, except for two of them that we quote in the next definition. (We mention that $N^{*}(L)$ satisfies these two additional properties; see [1].)

Definition 2.2 An $\mathcal{E}(2)$-algebra $A^{*}$ is nice if it satisfies the next two properties for all $x, x^{\prime} \in A$ of respective degrees $|x|$ and $\left|x^{\prime}\right|$ :

(i) $x \cup_{|x|} x=x$,

(ii) $\quad x \cup_{i} x^{\prime}=0$ if $i>\min \left(|x|,\left|x^{\prime}\right|\right)$.

Observe this useful property of nice $\mathcal{E}(2)$-algebras.

Lemma 2.3 Let $A$ be a nice $\mathcal{E}(2)$-algebra. If $a \in A^{d}$ and $b \in A^{d}$, we have

$$
a \cup_{d} b=b \cup_{d} a .
$$

Proof Property (ii) of Definition 2.2 and the Leibniz rule imply

$$
\begin{aligned}
\delta\left(a \cup_{d+1} b\right) & =0 \\
& =a \cup_{d} b+b \cup_{d} a+\delta a \cup_{d+1} b+a \cup_{d+1} \delta b \\
& =a \cup_{d} b+b \cup_{d} a .
\end{aligned}
$$

We recall now from [1] the construction of the tensor product of $\mathcal{E}(2)$-algebras. Let $\psi_{i}: \mathcal{E}(2) \otimes A_{i}^{\otimes 2} \rightarrow A_{i}$ be $\mathcal{E}(2)$-algebras for $i=0,1$. We use the diagonal $\mathcal{D}$ of $\mathcal{E}(2)$ for the construction of an $\mathcal{E}(2)$-action on the tensor product $A_{0} \otimes A_{1}$, as the following composite, denoted by $\Phi$, where Sh are the appropriate shuffle maps:

$\mathcal{E}(2) \otimes\left(A_{0} \otimes A_{1}\right)^{\otimes 2} \stackrel{\mathrm{Sh}}{\longrightarrow} \mathcal{E}(2) \otimes A_{0}^{\otimes 2} \otimes A_{1}^{\otimes 2} \stackrel{\mathcal{D} \otimes \mathrm{id} \otimes \mathrm{id}}{\longrightarrow} \mathcal{E}(2) \otimes \mathcal{E}(2) \otimes A_{0}^{\otimes 2} \otimes A_{1}^{\otimes 2}$

$$
A_{0} \otimes A_{1} \longleftarrow \psi_{0} \otimes \psi_{1} \quad \stackrel{\downarrow}{\downarrow} \mathcal{L}(2) \otimes A_{0}^{\otimes 2} \otimes \mathcal{E}(2) \otimes A_{1}^{\otimes 2}
$$


We have to verify that the map $\Phi$ satisfies the two conditions stated after Definition 2.1. Condition (2) is the compatibility with the differentials, which is direct here because $\Phi$ is the composite of maps that are compatible with the differentials. Thus, we are reduced to condition (1). Recall from the definition of the diagonal of $\mathcal{E}(2)$ :

$\mathcal{D}\left(e_{i}\right)=\sum_{j=0}^{i} e_{j} \otimes \tau^{j} . e_{i-j} \quad$ and $\quad \mathcal{D}\left(\tau_{i}\right)=\sum_{j=0}^{i} \tau . e_{j} \otimes \tau^{j+1} . e_{i-j}=\sum_{j=0}^{i} \tau_{j} \otimes \tau^{j} . \tau_{i-j}$.

A computation from the definition of $\Phi$ gives

$$
\Phi\left(\tau_{i} \otimes a_{0} \otimes a_{1} \otimes b_{0} \otimes b_{1}\right)=\sum_{j=0}^{i} \psi_{0}\left(\tau_{j} \otimes a_{0} \otimes b_{0}\right) \otimes \psi_{1}\left(\tau^{j} \cdot \tau_{i-j} \otimes a_{1} \otimes b_{1}\right)
$$

and

$$
\Phi\left(e_{i} \otimes b_{0} \otimes b_{1} \otimes a_{0} \otimes a_{1}\right)=\sum_{j=0}^{i} \psi_{0}\left(e_{j} \otimes b_{0} \otimes a_{0}\right) \otimes \psi_{1}\left(\tau^{j} . e_{i-j} \otimes b_{1} \otimes a_{1}\right) .
$$

If $j$ is even, we have

$$
\begin{aligned}
\psi_{1}\left(\tau^{j} . e_{i-j} \otimes b_{1} \otimes a_{1}\right) & =\psi_{1}\left(e_{i-j} \otimes b_{1} \otimes a_{1}\right) \\
& =\psi_{1}\left(\tau_{i-j} \otimes a_{1} \otimes b_{1}\right)=\psi_{1}\left(\tau^{j} \cdot \tau_{i-j} \otimes a_{1} \otimes b_{1}\right) .
\end{aligned}
$$

A similar computation in the case where $j$ is odd gives

$$
\Phi\left(\tau_{i} \otimes a_{0} \otimes a_{1} \otimes b_{0} \otimes b_{1}\right)=\Phi\left(e_{i} \otimes b_{0} \otimes b_{1} \otimes a_{0} \otimes a_{1}\right) .
$$

Now consider a family of $\mathcal{E}(2)$-algebras $\psi_{i}: \mathcal{E}(2) \otimes A_{i}^{\otimes 2} \rightarrow A_{i}$ with $i=0, \ldots, n$. As $\mathcal{D}: \mathcal{E}(2) \rightarrow \mathcal{E}(2) \otimes \mathcal{E}(2)$ is the diagonal of a bar resolution, it is a cochain map, it is coassociative [1], and we may iterate it as follows:

$$
\mathcal{D}^{2}\left(e_{i}\right)=\sum_{j=0}^{i} \mathcal{D}\left(e_{j}\right) \otimes \tau^{j} . e_{i-j}=\sum_{j=0}^{i} \sum_{k=0}^{j} e_{k} \otimes \tau^{k} . e_{j-k} \otimes \tau^{j} . e_{i-j}
$$

If we set $i_{1}=k, i_{2}=j-k$ and $i_{3}=i-j$, this last expression can be written as

$$
\mathcal{D}^{2}\left(e_{i}\right)=\sum_{\substack{\left(i_{1}, i_{2}, i_{3}\right) \\ i_{1}+i_{2}+i_{3}=i}} e_{i_{1}} \otimes \tau^{i_{1}} \cdot e_{i_{2}} \otimes \tau^{i_{1}+i_{2}} \cdot e_{i_{3}} .
$$

More generally, an induction gives

$$
\mathcal{D}^{n-1}\left(e_{i}\right)=\sum_{\substack{\left(i_{1}, \ldots, i_{n}\right) \\ i_{1}+\cdots+i_{n}=i}} e_{i_{1}} \otimes \tau^{i_{1}} \cdot e_{i_{2}} \otimes \cdots \otimes \tau^{i_{1}+\cdots+i_{n-1}} \cdot e_{i_{n}} .
$$


As in the previous case of two $\mathcal{E}(2)$-algebras, the action of $\mathcal{E}(2)$ on $\bigotimes_{i=0}^{n} A_{i}$ is obtained from appropriate shuffle maps and the iteration $\mathcal{D}^{n-1}$ of the diagonal. By using the notation in cup $_{i}$-products, this structure is defined as the map

$$
\mathcal{E}(2) \otimes\left(\bigotimes_{i=0}^{n} A_{i}\right)^{\otimes 2} \stackrel{\Phi}{\longrightarrow} \bigotimes_{i=0}^{n} A_{i}
$$

which sends the element $e_{i} \otimes\left(\bigotimes_{i=0}^{n} x_{i}\right) \otimes\left(\bigotimes_{i=0}^{n} y_{i}\right)$ to

$$
\sum_{\substack{\left(i_{1}, \ldots, i_{n}\right) \\ i_{1}+\cdots+i_{n}=i}}\left(x_{1} \cup_{i_{1}} y_{1}\right) \otimes\left(x_{2} \cup_{i_{2}}^{i_{1}} y_{2}\right) \otimes \cdots \otimes\left(x_{n} \cup_{i_{n}}^{i_{1}+\cdots+i_{n-1}} y_{n}\right),
$$

where we set, for $j \geq 0$,

$$
x \cup_{i}^{j} y= \begin{cases}x \cup_{i} y & \text { if } j \text { is even, } \\ y \cup_{i} x & \text { if } j \text { is odd. }\end{cases}
$$

Up to shuffle maps, $\Phi$ is obtained as a composite and tensor product of equivariant cochain maps; thus it satisfies the requirements of Definition 2.1. Moreover, as we establish below, the tensor product of nice $\mathcal{E}(2)$-algebras is a nice $\mathcal{E}(2)$-algebra.

Lemma 2.4 Any tensor product of nice $\mathcal{E}(2)$-algebras is a nice $\mathcal{E}(2)$-algebra for the product structure coming from the diagonal of $\mathcal{E}(2)$.

Proof By coassociativity of the diagonal of $\mathcal{E}(2)$, it is sufficient to reduce the proof to the case of the tensor product of two nice $\mathcal{E}(2)$-algebras $A$ and $B$.

Let $x=\sum_{k} a_{k} \otimes b_{k} \in(A \otimes B)^{d}$ and $x^{\prime}=\sum_{\ell} a_{\ell}^{\prime} \otimes b_{\ell}^{\prime} \in(A \otimes B)^{d^{\prime}}$ with $d \leq d^{\prime}$. We set $f=d+m$ with $m \geq 0$. One computes

$$
x \cup_{f} x^{\prime}=\sum_{f_{1}+f_{2}=f} \sum_{k, \ell}\left(a_{k} \cup_{f_{1}} a_{\ell}^{\prime}\right) \otimes\left(b_{k} \cup_{f_{2}}^{f_{1}} b_{\ell}^{\prime}\right) .
$$

If the element $\left(a_{k} \cup_{f_{1}} a_{\ell}^{\prime}\right) \otimes\left(b_{k} \cup_{f_{2}}^{f_{1}} b_{\ell}^{\prime}\right)$ of this sum is not equal to zero, we must have

$$
f_{1} \leq \min \left(\left|a_{k}\right|,\left|a_{\ell}^{\prime}\right|\right) \quad \text { and } \quad f_{2} \leq \min \left(\left|b_{k}\right|,\left|b_{\ell}^{\prime}\right|\right),
$$

which implies $f=f_{1}+f_{2}=d+m \leq\left|a_{k}\right|+\left|b_{k}\right|=d$ and $m=0$. We have established property (ii) of Definition 2.2. As for property (i), we consider

$$
x \cup_{d} x=\sum_{f_{1}+f_{2}=d} \sum_{k, k^{\prime}}\left(a_{k} \cup_{f_{1}} a_{k^{\prime}}\right) \otimes\left(b_{k} \cup_{f_{2}}^{f_{1}} b_{k^{\prime}}\right) .
$$


As above, if the element $\left(a_{k} \cup_{f_{1}} a_{k^{\prime}}\right) \otimes\left(b_{k} \cup_{f_{2}}^{f_{1}} b_{k^{\prime}}\right)$ of this sum is not equal to zero, we must have

$$
f_{1} \leq \min \left(\left|a_{k}\right|,\left|a_{k^{\prime}}\right|\right) \quad \text { and } \quad f_{2} \leq \min \left(\left|b_{k}\right|,\left|b_{k^{\prime}}\right|\right)
$$

Suppose $\min \left(\left|a_{k}\right|,\left|a_{k^{\prime}}\right|\right)=\left|a_{k}\right|$, then we have $\left|b_{k^{\prime}}\right| \leq\left|b_{k}\right|$, because $\left|a_{k}\right|+\left|b_{k}\right|=$ $\left|a_{k^{\prime}}\right|+\left|b_{k^{\prime}}\right|$, and also $d=\left|a_{k}\right|+\left|b_{k}\right|=f_{1}+f_{2} \leq\left|a_{k}\right|+\left|b_{k^{\prime}}\right|$; these imply $\left|b_{k}\right|=\left|b_{k^{\prime}}\right|$. Thus the nonzero elements of this sum must be of the form $\left(a_{k} \cup_{d-r} a_{k^{\prime}}\right) \otimes\left(b_{k} \cup_{r}^{d-r} b_{k^{\prime}}\right)$ with $\left|a_{k}\right|=\left|a_{k^{\prime}}\right|=d-r$ and $\left|b_{k}\right|=\left|b_{k^{\prime}}\right|=r$. With Lemma 2.3, if $a_{k} \neq a_{k^{\prime}}$, the term $\left(a_{k} \cup_{d-r} a_{k^{\prime}}\right) \otimes\left(b_{k} \cup_{r}^{d-r} b_{k^{\prime}}\right)$ also appears as $\left(a_{k^{\prime}} \cup_{d-r} a_{k}\right) \otimes\left(b_{k^{\prime}} \cup_{r}^{d-r} b_{k}\right)$. Their sum is equal to zero. With the same argument applied to the case $b_{k} \neq b_{k^{\prime}}$, we have reduced the previous expression to

$$
x \cup_{d} x=\sum_{k}\left(a_{k} \cup_{d-r} a_{k}\right) \otimes\left(b_{k} \cup_{r} b_{k}\right)=\sum_{k} a_{k} \otimes b_{k}=x,
$$

and Property (i) of Definition 2.2 is established.

We come back to the intersection setting and recall [4] that a perverse cochain complex is a functor defined on $\widehat{\mathcal{P}}^{n}$ with values in the category of cochain complexes. A functor from $\widehat{\mathcal{P}}_{\text {loose }}^{n}$ with values in the category of cochain complexes is called a generalized perverse cochain complex. For instance, if $\underline{K}$ is a filtered face set, the association $\bar{p} \mapsto \tilde{N}_{\bar{p}}^{*}(\underline{K})$ is a (generalized) perverse cochain complex, and this association is natural in $\underline{K}$.

Definition 2.5 Let $A_{\bullet}^{*}$ be a generalized perverse cochain complex. We denote by $\varphi_{\bar{p}}^{\bar{q}}: A_{\bar{p}}^{*} \rightarrow A_{\bar{q}}^{*}$ the morphism associated to $\bar{p} \leq \bar{q}$. A perverse $\mathcal{E}(2)$-algebra structure on $A_{\bullet}^{*}$ is a family of cochain maps $\psi_{\bar{p}, \bar{q}}: \mathcal{E}(2) \otimes A_{\bar{p}}^{*} \otimes A_{\bar{q}}^{*} \rightarrow A_{\bar{p}+\bar{q}}^{*}$ satisfying

(i) a compatibility condition with perversities: for any loose perversities $\bar{p}_{1}, \bar{q}_{1}$, $\bar{p}_{2}$ and $\bar{q}_{2}$, with $\bar{p}_{1} \leq \bar{p}_{2}$ and $\bar{q}_{1} \leq \bar{q}_{2}$, the following diagram is commutative:

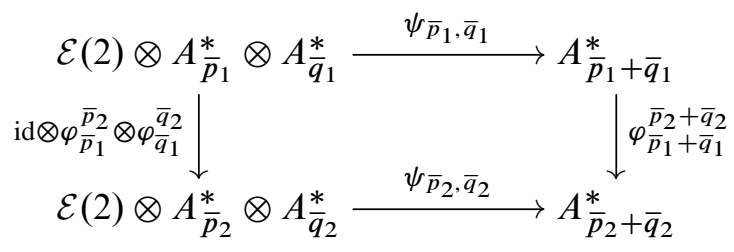

(ii) a $\Sigma_{2}$-equivariance as a map from $\mathcal{E}(2)$ to $\left(\operatorname{hom}\left(A_{\bar{p}}^{*} \otimes A_{\bar{q}}^{*}, A_{\bar{p}}^{*} \bar{q}\right)\right) \bar{p}, \bar{q}$ with the following $\Sigma_{2}$-action on the codomain: to any family $\eta_{\bar{p}, \bar{q}}: A_{\bar{p}}^{*} \otimes A_{\bar{q}}^{*} \rightarrow A_{\bar{p}}^{*} \bar{q}$, we associate the family $(\tau \eta)_{\bar{p}, \bar{q}}: A_{\bar{p}}^{*} \otimes A_{\bar{q}}^{*} \rightarrow A_{\bar{p}}^{*}+\bar{q}$ defined by $(\tau \eta)_{\bar{p}}, \bar{q}\left(x_{1} \otimes x_{2}\right)=$ $\eta \bar{q}, \bar{p}\left(x_{2} \otimes x_{1}\right)$. 
Equivalently, a perverse $\mathcal{E}(2)$-algebra structure on $A_{\bullet}^{*}$ is entirely determined by maps, called perverse cup c $_{i}$ products $\cup_{i}: A_{\bar{p}}^{r} \otimes A \frac{s}{q} \rightarrow A_{\bar{p}+\bar{q}}^{r+s}$, satisfying the previous Leibniz condition and the compatibility conditions with the poset structure of perversities. (The two settings are related by $x \cup_{i} y=\psi \bar{p}, \bar{q}\left(e_{i} \otimes x \otimes y\right)$.) Nice perverse $\mathcal{E}(2)$-algebras are defined as in Definition 2.2.

When $A_{\bullet}^{*}$ is a perverse cochain complex and the sum $\bar{p}+\bar{q}$ replaced by the sum of GMperversities $\bar{p} \oplus \bar{q}$ in Definition 2.5, we say that $A_{\bullet}^{*}$ is a GM-perverse $\mathcal{E}(2)$-algebra.

Let $\underline{K}$ be a filtered face set and $\sigma: \Delta=\Delta^{j_{0}} * \Delta^{j_{1}} * \cdots * \Delta^{j_{n}} \rightarrow \underline{K}$. With the tensor product of $\mathcal{E}(2)$-algebras recalled in (2) and the structure of nice $\mathcal{E}(2)$-algebras defined on the normalized cochain complex in [1], we get a structure of a nice $\mathcal{E}(2)$-algebra on the tensor product $\tilde{N}^{*}(\underline{K})_{\sigma}=N^{*}\left(c \Delta^{j_{0}}\right) \otimes \cdots \otimes N^{*}\left(c \Delta^{j_{n-1}}\right) \otimes N^{*}\left(\Delta^{j_{n}}\right)$. The next theorem establishes the compatibility of this structure with the perverse degrees.

Theorem $\mathbf{A}$ Let $\underline{K}$ be a filtered face set and $\bar{p}$ a loose perversity. The generalized perverse cochain complex $\bar{p} \mapsto \tilde{N}_{\bar{p}}^{*}(\underline{K})$ is a nice perverse $\mathcal{E}(2)$-algebra, natural in $\underline{K}$, for the filtered face maps.

Recall that a continuous map $f: X=\left(X_{j}\right)_{0 \leq j \leq n} \rightarrow Y=\left(Y_{j}\right)_{0 \leq j \leq n}$ between pseudomanifolds is a stratum preserving stratified map if, for any stratum $S^{\prime}$ of $Y^{\prime}, f^{-1}\left(S^{\prime}\right)$ is a union of strata of $X$, and $f^{-1}\left(Y_{n-\ell}\right)=X_{n-\ell}$ for any $\ell \geq 0$. As any stratumpreserving stratified map induces a filtered face set map, $\operatorname{ISing}^{\mathcal{F}}(X) \rightarrow \underline{\operatorname{ISing}}^{\mathcal{F}}(Y)$, (see [4, Example 1.5]) the next result is a direct consequence of Theorem $\overline{\mathrm{A}}$.

Corollary 2.6 Let $X$ be a pseudomanifold and $\bar{p}$ a loose perversity. The generalized perverse cochain complex $\left.\bar{p} \mapsto \tilde{N}_{\bar{p}}^{*} \underline{\operatorname{ISing}}^{\mathcal{F}}(X)\right)$ is a nice perverse $\mathcal{E}(2)$-algebra, natural in $X$ by stratum-preserving stratified maps.

Proof of Theorem A A cochain $c \in \tilde{N}^{*}(\underline{K)}$ associates to any simplex $\sigma: \Delta=$ $\Delta^{j_{0}} * \cdots * \Delta^{j_{n}} \rightarrow \underline{K}+$ an element $c_{\sigma} \in N^{*}\left(c \Delta^{j_{0}}\right) \otimes \cdots \otimes N^{*}\left(c \Delta^{j_{n-1}}\right) \otimes N^{*}\left(\Delta^{j_{n}}\right)$.

If we set $\left(c \cup_{i} c^{\prime}\right)_{\sigma}=c_{\sigma} \cup_{i} c_{\sigma}^{\prime}$, by naturality of the structure of an $\mathcal{E}(2)$-algebra on $N^{*}\left(c \Delta^{j_{0}}\right) \otimes \cdots \otimes N^{*}\left(c \Delta^{j_{n-1}}\right) \otimes N^{*}\left(\Delta^{j_{n}}\right)$, we get a global section $c \cup_{i} c^{\prime} \in \tilde{N}^{*}(\underline{K})$. More precisely, we have a $\Sigma_{2}$-equivariant cochain map

$$
\mathcal{E}(2) \otimes \tilde{N}^{*}(\underline{K})^{\otimes 2} \rightarrow \tilde{N}^{*}(\underline{K})
$$

entirely defined by $e_{i} \otimes c \otimes c^{\prime} \mapsto c \cup_{i} c^{\prime}$, which gives to $\tilde{N}^{*}(\underline{K})$ a structure of an $\mathcal{E}(2)$-algebra. The niceness of this structure is a direct consequence of Lemma 2.4.

The naturality in $\underline{K}$ comes from the naturality of the $\mathcal{E}(2)$-algebra structure on a tensor product, already mentioned, and from the naturality of the association $\underline{K} \mapsto \tilde{N}_{\bar{p}}^{*}(\underline{K})$; see [4, Proposition 1.36]. 
We now study the behavior of this structure with the perverse degree. The perversity degree is a local notion, so we let $c, c^{\prime} \in N^{*}\left(c \Delta^{j_{0}}\right) \otimes \cdots \otimes N^{*}\left(c \Delta^{j_{n-1}}\right) \otimes N^{*}\left(\Delta^{j_{n}}\right)$ with $j_{n} \geq 0$, and $\ell \in\{1, \ldots, n\}$ such that $\Delta^{j_{n-\ell}} \neq \varnothing$. We denote by $c_{n-\ell}$ and $c_{n-\ell}^{\prime}$ the respective restrictions of $c$ and $c^{\prime}$ to

$$
N^{*}\left(c \Delta^{j_{0}}\right) \otimes \cdots \otimes N^{*}\left(\Delta^{j_{n-\ell}} \times\{1\}\right) \otimes \cdots \otimes N^{*}\left(c \Delta^{j_{n-1}}\right) \otimes N^{*}\left(\Delta^{j_{n}}\right) .
$$

We decompose $c$ and $c^{\prime}$ as $c=\sum_{s=0}^{m} c_{0}^{s} \otimes \cdots \otimes c_{n}^{s}$ and $c^{\prime}=\sum_{t=0}^{m^{\prime}} c_{0}^{\prime t} \otimes \cdots \otimes c_{n}^{\prime t}$, and likewise their restrictions as

$c_{n-\ell}=\sum_{s=0}^{m} c_{0}^{s} \otimes \cdots \otimes \iota_{n-\ell}^{*} c_{n-\ell}^{s} \otimes \cdots \otimes c_{n}^{s} \quad$ and $\quad c_{n-\ell}^{\prime}=\sum_{t=0}^{m} c_{0}^{\prime t} \otimes \cdots \otimes \iota_{n-\ell}^{*} c_{n-\ell}^{\prime t} \otimes \cdots \otimes c_{n}^{\prime t}$, where $\iota_{n-\ell}^{*}$ is induced by the inclusion $\Delta^{j_{n-\ell}} \times\{1\} \hookrightarrow c \Delta^{j_{n-\ell}}$. By definition, we have

$$
\|c\|_{\ell}=\sup _{s}\left\{\left|c_{n-\ell+1}^{s} \otimes \cdots \otimes c_{n}^{s}\right| \text { such that } c_{0}^{s} \otimes \cdots \otimes \iota_{n-\ell}^{*} c_{n-\ell}^{s} \neq 0\right\} .
$$

Define $\hat{\iota}_{n-\ell}^{*}$ as follows:

$$
\begin{gathered}
N^{*}\left(c \Delta^{j_{0}}\right) \otimes \cdots \otimes N^{*}\left(c \Delta^{j_{n-\ell}}\right) \otimes \cdots \otimes N^{*}\left(c \Delta^{j_{n-1}}\right) \otimes N^{*}\left(\Delta^{j_{n}}\right) \\
\downarrow_{\hat{\imath}_{n-\ell}^{*}=\mathrm{id} \otimes \iota_{n-\ell}^{*} \otimes \mathrm{id}} \\
N^{*}\left(c \Delta^{j_{0}}\right) \otimes \cdots \otimes N^{*}\left(\Delta^{j_{n-\ell}} \times\{1\}\right) \otimes \cdots \otimes N^{*}\left(c \Delta^{j_{n-1}}\right) \otimes N^{*}\left(\Delta^{j_{n}}\right)
\end{gathered}
$$

As the $\operatorname{cup}_{i}$-product is natural, we have $\hat{\imath}_{n-\ell}^{*}\left(c \cup_{i} c^{\prime}\right)=\hat{\iota}_{n-\ell}^{*}(c) \cup_{i} \hat{\iota}_{n-\ell}^{*}\left(c^{\prime}\right)$.

- If $\hat{\iota}_{n-\ell}^{*}(c)=0$ or $\hat{\iota}_{n-\ell}^{*}\left(c^{\prime}\right)=0$, we have $\hat{\iota}_{n-\ell}^{*}(c) \cup_{i} \hat{\imath}_{n-\ell}^{*}\left(c^{\prime}\right)=0$, and thus

$$
\left\|c \cup_{i} c^{\prime}\right\|_{\ell}=-\infty \text {. }
$$

- Suppose now $\hat{\iota}_{n-\ell}^{*}(c) \neq 0$ and $\hat{\imath}_{n-\ell}^{*}\left(c^{\prime}\right) \neq 0$. By definition of the $\operatorname{cup}_{i}$-product, $\hat{\imath}_{n-\ell}^{*}(c) \cup_{i} \hat{\imath}_{n-\ell}^{*}\left(c^{\prime}\right)$ is a sum of tensor products whose elements are of two kinds:

(1) $c_{j}^{s} \cup_{f_{j}} c_{j}^{\prime t}$ with $j \neq n-\ell$, or

(2) $\hat{\imath}_{n-\ell}^{*}\left(c_{n-\ell}^{s}\right) \cup_{f_{n-\ell}} \hat{\imath}_{n-\ell}^{*}\left(c_{n-\ell}^{\prime t}\right)$.

As $\left|c_{j}^{s} \cup_{f_{j}} c_{j}^{\prime t}\right| \leq\left|c_{j}^{s}\right|+\left|c_{j}^{\prime t}\right|-f_{j}$, the cochain degree decreases and we obtain, for each $\ell$,

$$
\left\|c \cup_{i} c^{\prime}\right\|_{\ell} \leq\|c\|_{\ell}+\left\|c^{\prime}\right\|_{\ell}
$$

by definition of the perverse degree; see Definition 1.2. Therefore, we have

$$
\left\|c \cup_{i} c^{\prime}\right\| \leq\|c\|+\left\|c^{\prime}\right\|
$$


Now, the rule of Leibniz implies

$$
\left\|\delta\left(c \cup_{i} c^{\prime}\right)\right\| \leq \max \left(\|\delta c\|+\left\|c^{\prime}\right\|,\left\|\delta c^{\prime}\right\|+\|c\|,\|c\|+\left\|c^{\prime}\right\|\right) .
$$

Thus, if $\|c\| \leq \bar{p},\|\delta c\| \leq \bar{p},\left\|c^{\prime}\right\| \leq \bar{q}$ and $\left\|\delta c^{\prime}\right\| \leq \bar{q}$, we have $\left\|c \cup_{i} c^{\prime}\right\| \leq \bar{p}+\bar{q}$ and $\left\|\delta\left(c \cup_{i} c^{\prime}\right)\right\| \leq \bar{p}+\bar{q}$. This implies that the $\mathcal{E}(2)$-algebra structure on $\tilde{N}^{*}(\underline{K})$ induces equivariant cochain maps

$$
\mathcal{E}(2) \otimes \tilde{N}_{\bar{p}}^{*}(\underline{K}) \otimes \tilde{N}_{\bar{q}}^{*}(\underline{K}) \rightarrow \tilde{N}_{\bar{p}+\bar{q}}^{*}(\underline{K}) .
$$

This means that $\tilde{N}_{\bullet}^{*}(\underline{K})$ is a perverse $\mathcal{E}(2)$-algebra.

\section{Steenrod perverse squares}

From the existence of perverse $\operatorname{cup}_{i}$-products, we define Steenrod squares, as in the classical case. In the next statement, when $i>0$, the fact that the loose perversity image of $\mathrm{Sq}^{i}$ is $\mathcal{L}(\bar{p}, i)$, defined by $\mathcal{L}(\bar{p}, i)(\ell)=\min (2 \bar{p}(\ell), \bar{p}(\ell)+i)$, answers positively a conjecture of Goresky and Pardon; see [12, Conjecture 7.5]. More explicitly, we prove the existence of a dashed arrow which lifts the square $\mathrm{Sq}^{i}$ :

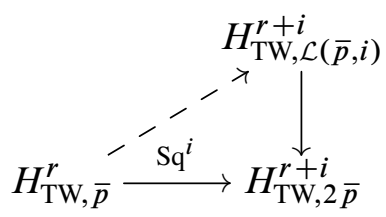

We still denote by $\mathrm{Sq}^{i}$ this lifting.

Theorem B Let $\underline{K}$ be a filtered face set and $\bar{p}, \bar{q}$ loose perversities The perverse cup $_{i}$-products induce natural perverse squares, defined by $\mathrm{Sq}^{i}(x)=x \cup_{|x|-i} x$ for $x \in H_{\mathrm{TW}, \bar{p}}^{|x|}(\underline{K})$, which satisfy the following properties:

(1) If $i<0$, then $\mathrm{Sq}^{i}(x)=0$.

(2) If $i \geq 0$, then we have

$$
\mathrm{Sq}^{i}: H_{\mathrm{TW}, \bar{p}}^{r}(\underline{K}) \rightarrow H_{\mathrm{TW}, \mathcal{L}(\bar{p}, i)}^{r+i}(\underline{K}),
$$

where $\mathcal{L}(\bar{p}, i)=\min (2 \bar{p}, \bar{p}+i)$, and

(i) $\mathrm{Sq}^{i}(x)=0$ if $i>|x|$;

(ii) $\mathrm{Sq}^{|x|}(x)=x^{2}$;

(iii) $\mathrm{Sq}^{0}=\mathrm{id}$; 
(iv) if $x \in H_{\mathrm{TW}, \bar{p}}^{|x|}(\underline{K})$ and $y \in H_{\mathrm{TW}, \bar{q}}^{|y|}(\underline{K})$, one has the (internal) Cartan formula

$$
\mathrm{Sq}^{i}(x \cup y)=\sum_{i_{1}+i_{2}=i} \operatorname{Sq}^{i_{1}}(x) \cup \operatorname{Sq}^{i_{2}}(y) \in H_{\mathrm{TW}, \bar{r}}^{|x|+|y|+i}(\underline{K}),
$$

with $\bar{r}=\min (2 \bar{p}+2 \bar{q}, \bar{p}+\bar{q}+i)$ and $\cup=\cup_{0}$;

(v) for any pair $(i, j)$ with $i<2 j$, one has the Adem relation

$$
\mathrm{Sq}^{i} \mathrm{Sq}^{j}=\sum_{k=0}^{[i / 2]}\left(\begin{array}{c}
j-k-1 \\
i-2 k
\end{array}\right) \mathrm{Sq}^{i+j-k} \mathrm{Sq}^{k}
$$

and $\mathrm{Sq}^{i} \mathrm{Sq}^{j}$ sends $H_{\mathrm{TW}, \bar{p}}^{*}$ into $H_{\mathrm{TW}, \bar{r}}^{*+i+j}$ with $\bar{r}=\min (4 \bar{p}, 2 \bar{p}+i, \bar{p}+i+j)$.

Before proving this theorem, we establish a technical property on the tensor product of two nice $\mathcal{E}(2)$-algebras, which is the keystone in the proof of Theorem B.

Lemma 3.1 Let $A$ and $B$ be two nice $\mathcal{E}(2)$-algebras and $A \otimes B$ their tensor product equipped with the $\mathcal{E}(2)$-algebra structure coming from the diagonal of $\mathcal{E}(2)$. Let $x, x^{\prime} \in A$ and $y, y^{\prime} \in B$ be such that $|x|+|y|=\left|x^{\prime}\right|+\left|y^{\prime}\right|=d,|y| \leq r$ and $\left|y^{\prime}\right| \leq r$. Then, for any $k \in\{0, \ldots, d-i\}$ such that $\left(x \cup_{d-k-i} x^{\prime}\right) \otimes\left(y \cup_{k}^{d-k-i} y^{\prime}\right) \neq 0$, we have $\left|y \cup_{k}^{d-k-i} y^{\prime}\right| \leq r+i$.

Proof Suppose $d-k-i$ even. If $\left(x \cup_{d-k-i} x^{\prime}\right) \otimes\left(y \cup_{k} y^{\prime}\right) \neq 0$, we must have $k \leq \min \left(|y|,\left|y^{\prime}\right|\right)$ and $d-k-i \leq \min \left(|x|,\left|x^{\prime}\right|\right)$, which implies

$$
d-i-\min \left(|x|,\left|x^{\prime}\right|\right) \leq k .
$$

Suppose $\min \left(|x|,\left|x^{\prime}\right|\right)=|x|$. Then we have

$$
|y|+\left|y^{\prime}\right|-d+i+\min \left(|x|,\left|x^{\prime}\right|\right)=|y|+\left|y^{\prime}\right|-(|x|+|y|)+i+|x|=\left|y^{\prime}\right|+i,
$$

which implies

$$
\left|y \cup_{k} y^{\prime}\right| \leq|y|+\left|y^{\prime}\right|-k \leq|y|+\left|y^{\prime}\right|-d+i+\min \left(|x|,\left|x^{\prime}\right|\right) \leq\left|y^{\prime}\right|+i \leq r+i .
$$

A similar argument gives the result in the case $\min \left(|x|,\left|x^{\prime}\right|\right)=\left|x^{\prime}\right|$. Also, the proof is analogous to the previous one if $d-k-i$ is odd, since $\left|y^{\prime} \cup_{k} y\right| \leq\left|y^{\prime}\right|+|y|-k$.

Directly from the definition of $\operatorname{cup}_{k}$-products, the inequalities $|y| \leq r$ and $\left|y^{\prime}\right| \leq r$ imply $\left|y \cup_{k} y^{\prime}\right| \leq 2 r$. Thus, the bound $\left|y \cup_{k} y^{\prime}\right| \leq r+i$ obtained in Lemma 3.1 is exactly what is needed for the proof of the Goresky-Pardon conjecture, as we show in the beginning of the next proof. 
Proof of Theorem B Let $i \geq 0$. From their definition as particular cup - $_{i}$-products, the Steenrod squares have their image in the intersection cohomology with loose perversity $2 \bar{p}$. We first prove that the loose perversity $2 \bar{p}$ can be replaced by $\mathcal{L}(\bar{p}, i)$. We take over the arguments and the method used at the end of the proof of Theorem A by considering a cocycle $c \in N^{*}\left(c \Delta^{j_{0}}\right) \otimes \cdots \otimes N^{*}\left(c \Delta^{j_{n-1}}\right) \otimes N^{*}\left(\Delta^{j_{n}}\right), \ell \in\{1, \ldots, n\}$ such that $\Delta^{j_{n-\ell}} \neq \varnothing$, and the restriction $c_{n-\ell}$ of $c$ to

$$
N^{*}\left(c \Delta^{j_{0}}\right) \otimes \cdots \otimes N^{*}\left(\Delta^{j_{n-\ell}} \times\{1\}\right) \otimes \cdots \otimes N^{*}\left(c \Delta^{j_{n-1}}\right) \otimes N^{*}\left(\Delta^{j_{n}}\right) .
$$

First observe that, by naturality, we have $\left(c \cup_{|c|-i} c\right)_{n-\ell}=c_{n-\ell} \cup_{\left|c_{n-\ell}\right|-i} c_{n-\ell}$.

- If $c_{n-\ell}=0$, we have $\left(c \cup_{|c|-i} c\right)_{n-\ell}=0$ and $\left\|c \cup_{|c|-i} c\right\|_{\ell}=-\infty$.

- If $c_{n-\ell} \neq 0$, then we let $A=N^{*}\left(c \Delta^{j_{0}}\right) \otimes \cdots \otimes N^{*}\left(\Delta^{j_{n-\ell}} \times\{1\}\right)$ and let $B=N^{*}\left(c \Delta^{j_{n-\ell+1}}\right) \otimes \cdots \otimes N^{*}\left(c \Delta^{j_{n-1}}\right) \otimes N^{*}\left(\Delta^{j_{n}}\right)$, and so we decompose $c_{n-\ell}$ in a canonical form, $c_{n-\ell}=\sum_{s} c_{n-\ell}^{\prime s} \otimes c_{n-\ell}^{\prime \prime s} \in A \otimes B$. Now, using Lemma 3.1, we know that

$$
\left(c_{n-\ell}^{\prime s} \cup_{\left|c_{n-\ell}\right|-k-i} c_{n-\ell}^{\prime t}\right) \otimes\left(c_{n-\ell}^{\prime \prime s} \cup_{k}^{\left|c_{n-\ell}\right|-k-i} c_{n-\ell}^{\prime \prime t}\right) \neq 0
$$

implies

$$
\left|c_{n-\ell}^{\prime \prime s} \cup_{k}^{\left|c_{n-\ell}\right|-k-i} c_{n-\ell}^{\prime \prime t}\right| \leq \bar{p}(\ell)+i
$$

for any pair of indices $(s, t)$ in the writing of $c_{n-\ell}$. This implies $\left\|c \cup_{|c|-i} c\right\| \leq$ $\bar{p}+i$, as stated.

The condition on the perversity of the differential of $c \cup_{|c|-i} c$ is immediate here because $c$ is a cocycle, and the naturality follows from the fact that the lifting already exists at the level of the spaces of cocycles.

The properties (1), (2i), (2ii) and (2iii) are a direct consequence of Theorem A and [18, Section 5].

Let $A$ and $B$ be two nice $\mathcal{E}(2)$-algebras. By definition of the diagonal action of $\mathcal{E}(2)$ on the tensor product, we have a Cartan external formula

$$
\mathrm{Sq}^{i}(a \otimes b)=\sum_{i_{1}+i_{2}=i} \mathrm{Sq}^{i_{1}}(a) \otimes \mathrm{Sq}^{i_{2}}(b)
$$

for $a \in A$ and $b \in B$. In our case, each factor, $A$ and $B$, satisfies the Cartan internal formula. Therefore, the Cartan internal formula on $A \otimes B$ is a direct consequence of 
the next equalities:

$\mathrm{Sq}^{i}\left((a \otimes b) \cup\left(a^{\prime} \otimes b^{\prime}\right)\right)$

$$
\begin{aligned}
& ={ }_{(1)} \mathrm{Sq}^{i}\left(\left(a \cup a^{\prime}\right) \otimes\left(b \cup b^{\prime}\right)\right) \\
& ={ }_{(2)} \sum_{i_{1}+i_{2}=i} \operatorname{Sq}^{i_{1}}\left(a \cup a^{\prime}\right) \otimes \operatorname{Sq}^{i_{2}}\left(b \cup b^{\prime}\right) \\
& =(3) \sum_{j_{1}+j_{2}+k_{1}+k_{2}=i}\left(\mathrm{Sq}^{j_{1}}(a) \cup \mathrm{Sq}^{j_{2}}\left(a^{\prime}\right)\right) \otimes\left(\mathrm{Sq}^{k_{1}}(b) \cup \mathrm{Sq}^{k_{2}}\left(b^{\prime}\right)\right),
\end{aligned}
$$

and

$$
\begin{aligned}
\sum_{i_{1}+i_{2}=i} \operatorname{Sq}^{i_{1}}(a \otimes b) & \cup \operatorname{Sq}^{i_{2}}\left(a^{\prime} \otimes b^{\prime}\right) \\
& ={ }_{(2)} \sum_{s_{1}+s_{2}+t_{1}+t_{2}=i}\left(\operatorname{Sq}^{s_{1}}(a) \otimes \operatorname{Sq}^{s_{2}}(b)\right) \cup\left(\operatorname{Sq}^{t_{1}}\left(a^{\prime}\right) \otimes \operatorname{Sq}^{t_{2}}\left(b^{\prime}\right)\right) \\
& =(1) \sum_{s_{1}+s_{2}+t_{1}+t_{2}=i}\left(\operatorname{Sq}^{s_{1}}(a) \cup \operatorname{Sq}^{t_{1}}\left(a^{\prime}\right)\right) \otimes\left(\mathrm{Sq}^{s_{2}}(b) \cup \operatorname{Sq}^{t_{2}}\left(b^{\prime}\right)\right),
\end{aligned}
$$

where $={ }_{(1)}$ comes from the definition of the cup-product on a tensor product, $={ }_{(2)}$ from the application of the Cartan external formula and $={ }_{(3)}$ from the Cartan internal formula on each factor.

For Adem's formula (2v), we recall some properties in order to track the perversity conditions. The classical proof uses the bar resolution $\mathcal{E}(4)$ of $\mathbb{F}_{2}$ as a $\Sigma_{4}$-module, and the existence of a $\Sigma_{4}$-equivariant cochain map $\mathcal{E}(4) \otimes N^{*}(L)^{\otimes 4} \rightarrow N^{*}(L)$ for any simplicial set $L$, called an $\mathcal{E}(4)$-algebra. As these objects appear just in this part of proof, we do not recall them in detail, referring to [1, Section 1]. We mention only the points related to the control of perversities.

Denote by $\omega: \mathcal{E}(2) \otimes \mathcal{E}(2) \otimes \mathcal{E}(2) \rightarrow \mathcal{E}(4)$ the cochain map induced by the wreath product $\Sigma_{2} \times \Sigma_{2} \times \Sigma_{2} \rightarrow \Sigma_{4}$. Let $A$ be an $\mathcal{E}(2)$ and an $\mathcal{E}(4)$-algebra whose structure maps are respectively denoted by $\psi_{2}$ and $\psi_{4}$. By definition, we say that $A$ is an Adem-object [18] if there is a commutative diagram

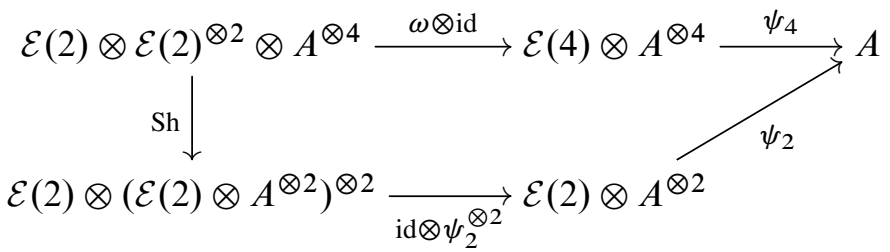

where Sh is the appropriate shuffle map. 
Let $\Delta=\Delta^{j_{0}} * \cdots * \Delta^{j_{n}}$ and $A=N^{*}\left(c \Delta^{j_{0}}\right) \otimes \cdots \otimes N^{*}\left(c \Delta^{j_{n-1}}\right) \otimes N^{*}\left(\Delta^{j_{n}}\right)$. Because $N^{*}(L)$ is an Adem-object for any simplicial set $L$, and because the tensor product of two nice $\mathcal{E}(2)$-algebras which are Adem-objects is an Adem-object [18, Lemma 4.2, page 174], $A$ is an Adem-object.

In Theorem A, we prove that $\psi_{2}$ restricts to a map $\mathcal{E}(2) \otimes A_{\bar{p}} \otimes A_{\bar{q}} \rightarrow A_{\bar{p}+\bar{q}}$. Exactly the same argument can be used for $\psi_{4}$, replacing $c \cup_{i} c^{\prime}$ by $\psi_{4}\left(\alpha_{i} \otimes c_{1} \otimes c_{2} \otimes c_{3} \otimes c_{4}\right)$ for each $\alpha_{i} \in \mathcal{E}(4)$ in the last part of the proof of Theorem A. Thus $\psi_{4}$ restricts to

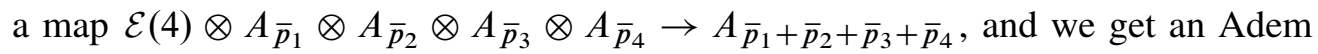
formula for intersection cohomology.

Successive applications of Lemma 3.1 show that the nonzero terms in the right-hand side of the Adem relation belong to intersection cohomology in perversities less than, or equal to, $\min (4 \bar{p}, 2 \bar{p}+2 j, 2 \bar{p}+i, \bar{p}+i+j) \leq \min (4 \bar{p}, 2 \bar{p}+i, \bar{p}+i+j)$, since $i<2 j$. The same argument applied to the left-hand side implies that the nonzero terms belong also to intersection cohomology in the same range of perversities.

Remark 3.2 Previous definitions and results can be adapted to the context of GMperversities. By restricting to GM-perversities $\bar{p}$ and $\bar{q}$ such that $\bar{p}+\bar{q} \leq \bar{t}$, the cup $_{i}$-products are defined by

$$
\cup_{i}: A_{\bar{p}}^{r} \otimes A_{\bar{q}}^{s} \rightarrow A_{\bar{p} \oplus \bar{q}}^{r+s-i},
$$

where the sum $\bar{p} \oplus \bar{q}$ is taken in the lattice $\mathcal{P}^{n}$; see [14] or [4, Section 2.1]. The Steenrod squares introduced in Section 3,

$$
\mathrm{Sq}^{i}: H_{\mathrm{TW}, \bar{p}}^{r} \rightarrow H_{\mathrm{TW}, \bar{r}}^{r+i},
$$

are therefore defined for GM-perversities $\bar{p}$ and $\bar{r}$ such that $\min (2 \bar{p}, \bar{p}+i) \leq \bar{r}$.

\section{Comparison with Goresky's construction}

As this section is concerned with isomorphisms between different definitions of Steenrod squares in intersection cohomology, in some crucial points, we keep all the information in the notation of cohomology groups.

In [11] (see also [2]), the intersection cohomology on a pseudomanifold $X$ is introduced by the use of a sheaf due to Deligne. The Deligne sheaf $\mathcal{P}_{\bar{p}}$ is defined by a sequence of truncations starting from the constant sheaf on $X_{n} \backslash X_{n-2}$. As we are not using this specific construction, we do not recall it, sending the reader to the previous references. 
In [13], Goresky has already defined Steenrod squares $\mathrm{Sq}_{\mathrm{G}}^{i}$ on the intersection cohomology $H^{*}\left(X ; \mathcal{P}_{\bar{p}}\right)$ of a topological pseudomanifold $X$ in the case of a GM-perversity $\bar{p}$. In this section, we prove that the two Steenrod squares, $\mathrm{Sq}^{i}$ and $\mathrm{Sq}_{\mathrm{G}}^{i}$, coincide.

Recall the filtered face set $\operatorname{ISing}^{\mathcal{F}}(X)$ introduced in Remark 1.7. The next result connects Goresky's definition of Steenrod squares on $H^{*}\left(X ; \mathcal{P}_{\bar{p}}\right)$ to our definition of Steenrod squares on the TW-cohomology of the filtered face set $\operatorname{ISing}^{\mathcal{F}}(X)$, denoted $H_{\mathrm{TW}, \bar{p}}^{*}(X)$.

Theorem C Let $X$ be an $n$-dimensional topological pseudomanifold. For any GMperversity $\bar{q}$, there exists an isomorphism $\theta_{\bar{q}}^{*}: H_{\mathrm{TW}, \bar{q}}^{*}(X) \rightarrow H^{*}(X ; \mathcal{P} \bar{q})$. Moreover, if $\bar{p}$ is a GM-perversity such that $2 \bar{p} \leq \bar{t}$, then the following diagram commutes:

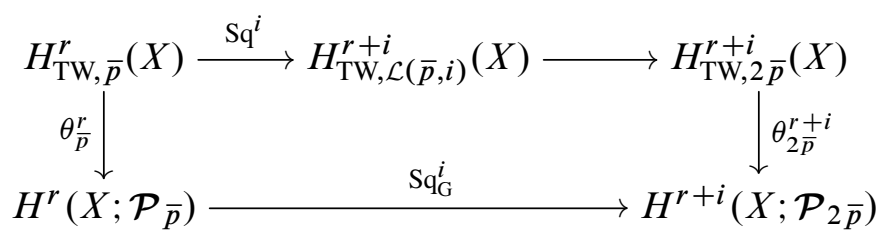

The previous statement implies that

$$
\theta_{\mathcal{L}(\bar{p}, i)}^{r+i} \circ \mathrm{Sq}^{i} \circ\left(\theta_{\bar{p}}^{r}\right)^{-1}: H^{r}\left(X ; \mathcal{P}_{\bar{p}}\right) \rightarrow H^{r+i}\left(X ; \mathcal{P}_{\mathcal{L}(\bar{p}, i)}\right)
$$

is a lift of the Steenrod squares defined by Goresky,

$$
\mathrm{Sq}_{\mathrm{G}}^{i}: H^{r}\left(X ; \mathcal{P}_{\bar{p}}\right) \rightarrow H^{r+i}\left(X ; \mathcal{P}_{2 \bar{p}}\right)
$$

Therefore the Goresky-Pardon conjecture has a positive answer.

From the functor $\tilde{N}^{*}$, we define a presheaf on $X$ by

$$
\left.\operatorname{IN}_{\bar{p}}^{*}(U)=\tilde{N}_{\bar{p}}^{*} \underline{\operatorname{ISing}}^{\mathcal{F}}(U)\right)
$$

for any open set $U$ of $X$. Denote by $\operatorname{Cov}(U)$ the directed set of open covers of $U$, ordered by inclusions. For any $\mathcal{U} \in \operatorname{Cov}(U), \operatorname{ISing}^{\mathcal{F}, \mathcal{U}}(U)$ is the subfiltered face set of $\operatorname{ISing}^{\mathcal{F}}(U)$ whose elements have a support included in an element of $\mathcal{U}$. The sheafification of $\operatorname{IN}_{\bar{p}}^{*}$ is given by

$$
\mathbf{I N}_{\bar{p}}^{*}(U)=\lim _{\mathcal{U} \in \operatorname{Cov}(U)} \tilde{N}_{\bar{p}}^{*}\left(\underline{\operatorname{ISing}}^{\mathcal{F}, \mathcal{U}}(U)\right)
$$

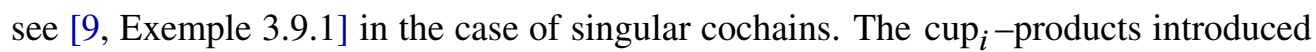
in Section 3 on $\tilde{N}_{\bullet}^{*}\left(\underline{\operatorname{ISing}}^{\mathcal{F}, \mathcal{U}}(U)\right)$ induce $\operatorname{cup}_{i}$-products on $\mathbf{I N}_{\bullet}^{*}(U)$ by definition of the last one as a direct limit. 
Theorem $\mathrm{C}$ is a direct consequence of Lemmas 4.1 and 4.3. First, we connect the definition of Steenrod squares on $\underline{\operatorname{Sing}}^{\mathcal{F}}(X)$ with a definition involving the sheaf IN* on $X$.

Lemma 4.1 For any $n$-dimensional topological pseudomanifold $X$ and any GMperversity $\bar{p}$, we have a commutative diagram:

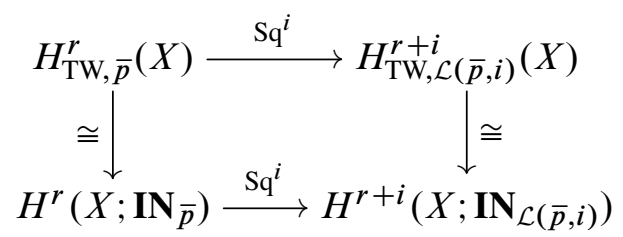

The vertical maps are isomorphisms induced by the canonical map IN* $\rightarrow \mathbf{I N}_{\bullet}^{*}$.

Proof For any $\mathcal{U} \in \operatorname{Cov}(X)$, the restriction map

$$
r_{\mathcal{U}}: \operatorname{IN}_{\bar{p}}^{*}(X) \rightarrow \tilde{N}_{\bar{p}}^{*}\left(\underline{\operatorname{ISing}}^{\mathcal{F}, \mathcal{U}}(X)\right)
$$

is compatible with the inclusions of open covers. This gives the morphism

$$
\operatorname{IN}_{\bar{p}}^{*}(X) \rightarrow \Gamma\left(X, \mathbf{I N}_{\bar{p}}^{*}\right):=\lim _{\mathcal{U} \in \operatorname{Cov}(X)} \tilde{N}_{\bar{p}}^{*}\left(\underline{\operatorname{ISing}}^{\mathcal{F}, \mathcal{U}}(X)\right)
$$

induced by the canonical map $\mathrm{IN}_{\bullet}^{*} \rightarrow \mathbf{I N}_{\bullet}^{*}$. By taking the direct limit of the quasiisomorphisms of Lemma 4.2, we get an isomorphism

$$
\left.H^{*}\left(\lim _{\mathcal{U} \in \operatorname{Cov}(U)} \tilde{N}_{\bar{p}}^{*}\left(\underline{\operatorname{ISing}}^{\mathcal{F}, \mathcal{U}}(U)\right)\right) \cong H^{*}\left(\tilde{N}_{\bar{p}}^{*} \underline{\operatorname{ISing}}^{\mathcal{F}}(U)\right)\right)=H_{\mathrm{TW}, \bar{p}}^{*}(U) .
$$

In a second step, by following the lines of [9, Exemple 3.9.1.], we prove that the sheaf $\mathbf{I N}_{\bar{p}}^{*}$ is soft. The elements of $\operatorname{IN}_{\overline{0}} \frac{0}{}(U)$ are $\overline{0}$-admissible vertices; they are the vertices of the regular part and the map $N^{0}(U) \rightarrow \mathrm{IN}_{0}^{0}(U)$ can be considered as the restriction to the regular part. Also, in this degree 0 , the presheaves $N^{0}$ and $\mathrm{IN}_{0}^{0}$ are clearly sheaves, and $N^{0}(U) \rightarrow \operatorname{IN} \frac{0}{0}(U)$ is a morphism of sheaves of rings. Observe also that $\operatorname{IN}_{\bar{p}}^{*}(U)$ is an $\operatorname{IN} \frac{0}{0}(U)$-module for the cup-product. As the sheaf $N^{0}$ is soft, and as (see [9, Théorème 3.7.1.]) any sheaf of modules over a soft sheaf of rings is soft, we deduce the softness of $\mathbf{I} \mathbf{N}_{\bar{p}}^{*}$. Thus the hypercohomology is the cohomology of the space of sections of the sheaf, and we get a series of isomorphisms

$$
H^{*}\left(X ; \mathbf{I N}_{\bar{p}}^{*}\right) \cong H^{*}\left(\Gamma\left(X, \mathbf{I N}_{\bar{p}}^{*}\right)\right) \cong H^{*}\left(\tilde{N}_{\bar{p}}^{*}\left(\underline{\operatorname{ISing}}^{\mathcal{F}}(X)\right)\right)=H_{\mathrm{TW}, \bar{p}}^{*}(X)
$$


By definition of the $\operatorname{cup}_{i}$-products on $\mathbf{I N}_{\bullet}^{*}$, the following diagram commutes:

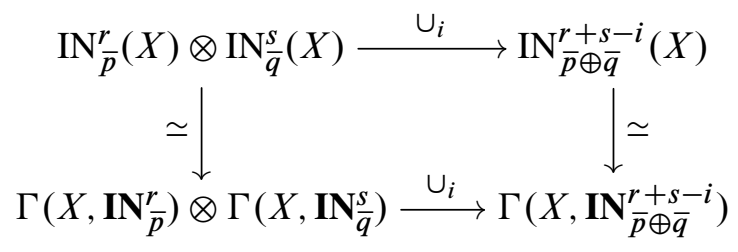

With the properties already established, the vertical maps are quasi-isomorphisms induced by the canonical map $\mathrm{IN}_{\bullet}^{*} \rightarrow \mathbf{I N}_{\bullet}^{*}$. The stated result is now a consequence of the definition of Steenrod squares from cup $_{i}$-products.

Lemma 4.2 Let $X$ be an $n$-dimensional pseudomanifold and $\mathcal{U}$ an open cover of $X$. The canonical inclusion $\iota: \operatorname{ISing}^{\mathcal{F}, \mathcal{U}}(X) \rightarrow \operatorname{ISing}^{\mathcal{F}}(X)$ induces an isomorphism in intersection cohomology for any GM-perversity $\bar{p}$.

Proof With Proposition 1.5 , we can replace $\tilde{N}^{*}(-)$ by the blow-up $\widetilde{C}^{*}(-)$ already studied in [4]. Let $\bar{q}$ be the GM-perversity defined by $\bar{p}(k)+\bar{q}(k)=k-2$. Recall from [4, Theorem B] that, for any filtered face set $\underline{K}$, there exists a quasi-isomorphism eval: $\widetilde{C}_{\bar{p}}^{*}(\underline{K}) \rightarrow \operatorname{hom}\left(C_{*}^{\mathrm{GM}, \bar{q}}(\underline{K}), \mathbb{F}_{2}\right)$, defined as follows: for any $\Phi \in \widetilde{C}_{\bar{p}}^{*}(\underline{K})$ and $\sigma: \Delta^{j_{0}} * \cdots * \Delta^{j_{n}} \rightarrow \underline{K}$, we have

$$
\Phi_{\sigma}=\sum_{j} \Phi_{0, \sigma, j} \otimes \cdots \otimes \Phi_{n, \sigma, j} \in C^{*}\left(c \Delta^{j_{0}}\right) \otimes \cdots \otimes C^{*}\left(\Delta^{j_{n}}\right),
$$

and we set

$$
\operatorname{eval}(\Phi)(\sigma)=\sum_{j} \Phi_{0, \sigma, j}\left(\left[c \Delta^{j_{0}}\right]\right) \cdots \Phi_{n, \sigma, j}\left(\left[\Delta^{j_{n}}\right]\right)
$$

where [-] is the maximal simplex. Applying to $\underline{K}=\underline{\operatorname{ISing}}^{\mathcal{F}}(X)$ and $\underline{K}=\operatorname{ISing}^{\mathcal{F}, \mathcal{U}}(X)$, we get the following diagram, whose commutativity follows directly from the definitions of the maps:

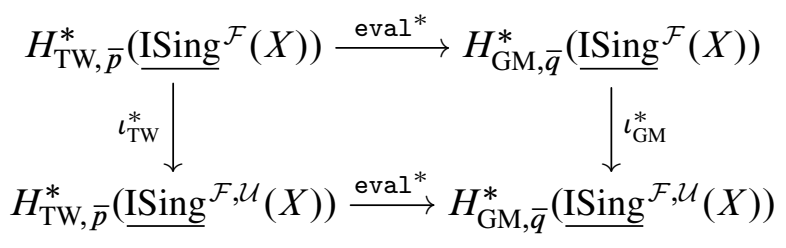

We know that the two evaluation maps eval* are quasi-isomorphisms, and we have to prove that the map $\iota_{\mathrm{TW}}^{*}$, induced by the inclusion $\iota$, is an isomorphism. With the 
commutativity of the previous diagram and the fact that the homology is over a field, it is sufficient to prove that

$$
\iota_{\mathrm{GM}, *}: H_{*}^{\mathrm{GM}, \bar{q}}\left(\underline{\mathrm{ISing}}^{\mathcal{F}, \mathcal{U}}(X)\right) \rightarrow H_{*}^{\mathrm{GM}, \bar{q}}\left(\underline{\operatorname{ISing}}^{\mathcal{F}}(X)\right)
$$

is an isomorphism. Set $C_{*}^{\bar{q}}(X)=C_{*}^{\mathrm{GM}, \bar{q}}\left(\operatorname{ISing}^{\mathcal{F}}(X)\right)$. Recall from [4, Lemma A.16] the existence of a chain map which is the classical subdivision sd: $C_{*}^{\bar{q}}(X) \rightarrow C_{*}^{\bar{q}}(X)$, and for any integer $m$, the existence of a homomorphism $T: C_{*}^{\bar{q}}(X) \rightarrow C_{*+1}^{\bar{q}}(X)$ such that $\partial T+T \partial=\mathrm{id}-\mathrm{sd}^{\mathrm{m}}$. By construction, for any element $c \in C_{*}^{\bar{q}}(X)$, there is an integer $m$ such that $\operatorname{sd}^{m} c \in C_{*}^{\mathrm{GM}}, \bar{q}\left(\operatorname{ISing}^{\mathcal{F}, \mathcal{U}}(X)\right)$. Moreover, if $c \in C_{*}^{\mathrm{GM}, \bar{q}}\left(\underline{\operatorname{ISing}}{ }^{\mathcal{F}, \mathcal{U}}(X)\right)$,

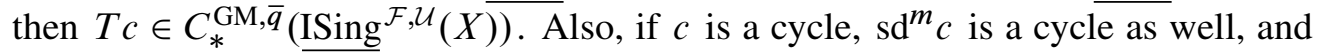
the two homology classes $[c]$ and $\left[\mathrm{sd}^{m} c\right]$ are equal. This implies the surjectivity and injectivity of $\iota_{\mathrm{GM}, *}$ through a classical argument.

The second step in the proof of Theorem $\mathrm{C}$ is the comparison of the two definitions of Steenrod squares, respectively associated to the sheaf $\mathbf{I N}_{\bullet}^{*}$ and to the Deligne sheaf $\mathcal{P}_{\bullet}^{*}$.

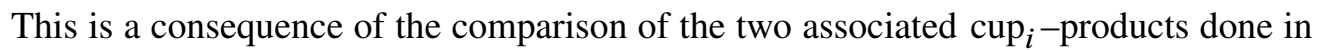
the next lemma.

Lemma 4.3 Let $X$ be an $n$-dimensional topological pseudomanifold, and let $\bar{p}$ and $\bar{q}$ be GM-perversities such that $\bar{p} \oplus \bar{q} \leq \bar{t}$, where $\bar{p} \oplus \bar{q}$ is the smallest GM-perversity $\bar{r}$ such that $\bar{p}+\bar{q} \leq \bar{r}$. Then for any $i$, there is a commutative square

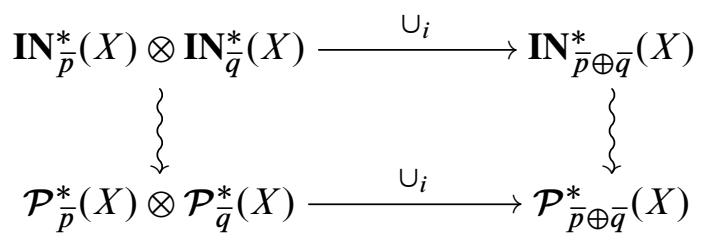

in the derived category of sheaves on $X$, linking the two cup $p_{i}$-products, such that vertical arrows are isomorphisms.

Proof Let $\mathbf{S}^{*}$ be a differential graded sheaf on the pseudomanifold $X$. We denote by $\mathbf{S}_{k}^{*}$ the restriction of $\mathbf{S}^{*}$ to the open set $X \backslash X_{n-k}$ for $k \in\{2, \ldots, n+1\}$. Recall the conditions (AX1) of $[2,2.3]$ :

(a) $\mathbf{S}^{*}$ is bounded, $\mathbf{S}^{i}=0$ for $i<0$ and $\mathbf{S}_{2}^{*}$ is quasi-isomorphic to the ordinary singular cohomology.

(b) For any $k \in\{2, \ldots, n\}$ and any $x \in X_{n-k} \backslash X_{n-k-1}$, we have $\mathcal{H}^{i}(\mathbf{S})_{x}=0$ if $i>\bar{p}(k)$.

(c) The attachment map $\alpha_{k}: \mathbf{S}_{k+1}^{*} \rightarrow R i_{k^{*}} \mathbf{S}_{k}^{*}$, induced by the canonical inclusion $X \backslash X_{n-k} \rightarrow X \backslash X_{n-k-1}$, is a quasi-isomorphism up to $\bar{p}(k)$. 
If $\mathbf{S}^{*}$ is soft, from [20, Remark 2.3.], we may replace condition (c) by the following equivalent one:

(c') For any $k \in\{2, \ldots, n\}, j \leq \bar{p}(k)$ and $x \in X_{n-k} \backslash X_{n-k-1}$, the restriction map induces an isomorphism

$$
\lim _{U_{x}} H^{j}\left(\Gamma\left(U_{x} ; \mathbf{S}^{*}\right)\right) \stackrel{\cong}{\longrightarrow} \lim _{U_{x}} H^{j}\left(\Gamma\left(U_{x} \backslash X_{n-k} ; \mathbf{S}^{*}\right)\right),
$$

where $U_{x}$ varies into a cofinal family of neighborhoods of $x$ in $X \backslash X_{n-k-1}$.

On the regular part, the sheaf $\mathbf{I N}_{\mathbf{0}}^{*}$ is the sheafification of $N^{*}$ and thus computes the singular cohomology. Therefore, condition (a) is satisfied for $\mathbf{I N}^{*}$. In order to prove that the sheaf $\mathbf{I N}_{\bullet}^{*}$ satisfies the axioms (b) and $\left(\mathrm{c}^{\prime}\right)$, we use the isomorphism established in Lemma 4.1,

$$
H^{*}\left(X ; \mathbf{I N}_{\bullet}\right) \cong H_{\mathrm{TW}, \bullet}^{*}(X) .
$$

Let $x \in X_{n-k} \backslash X_{n-k-1}$. The cohomology $\mathcal{H}^{*}\left(\mathbf{I N}_{\bullet}\right)_{x}$ is determined by the following isomorphisms:

$$
\mathcal{H}^{*}\left(\mathbf{I} \mathbf{N}_{\bullet}\right)_{x}=\underline{\lim }_{U_{x}} H^{*}\left(\Gamma\left(U_{x} ; \mathbf{I N}_{\bullet}\right)\right) \cong \underline{\lim }_{U_{x}} H_{\mathrm{TW}, \bullet}^{*}\left(U_{x}\right),
$$

where the direct limits are taken over the open neighborhoods $U_{x}$ of $x$. (The first equality is the definition of the stalk at a point.) Moreover, these limits can also be obtained from a restriction to a cofinal family of trivializing open neighborhoods $U_{x} \cong \mathbb{R}^{n-k} \times c L$, where $L$ is the link of $x$. Thus axiom (b) now follows from $H_{\mathrm{TW}, \bar{p}}^{*}\left(\mathbb{R}^{n-k} \times c L\right)=H_{\mathrm{TW}, \bar{p}}^{*}(c L)=0$ if $*>\bar{p}(k)$; see [4, Corollary 1.47].

The verification of $\left(\mathrm{c}^{\prime}\right)$ is quite similar. As in [20, Proof of Theorem 7.1.], we are reduced to analyzing the map

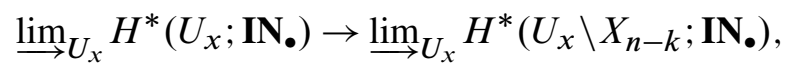

where the direct limit is taken over a cofinal family $U_{x} \cong \mathbb{R}^{n-k} \times c L$ of trivializing open neighborhoods of $x$. We consider the following commutative diagram, whose horizontal maps are induced by the canonical inclusions and vertical maps are isomorphisms:

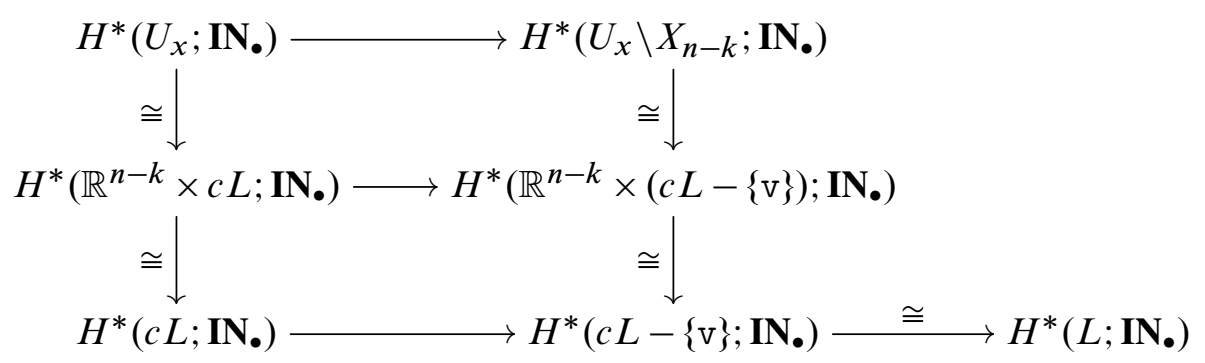


Finally, we note that the composite at the bottom is an isomorphism when $* \leq \bar{p}(k)$, as shows the classical computation of the intersection cohomology of a cone. Modulo the vertical isomorphisms, this is exactly the axiom $\left(\mathrm{c}^{\prime}\right)$.

Therefore, the sheaf IN* satisfies conditions (AX1), and by [2, Theorem 2.5], there exists a quasi-isomorphism between $\mathbf{I N}_{\bullet}^{*}$ and $\mathcal{P}_{\bullet}^{*}$; see also [11]. As a consequence, these two sheaves have a common injective resolution, and we may apply to it the uniqueness of $\operatorname{cup}_{i}$-products established by Goresky in [13, Proposition 3.6].

From the previous results on cup $_{i}$-products, we get an isomorphism of algebras of cohomology with coefficients in $\mathbb{F}_{2}$.

Corollary 4.4 If $X$ is an $n$-dimensional pseudomanifold, there are isomorphisms of perverse algebras

$$
H_{\mathrm{TW}, \bullet}^{*}(X) \cong H^{*}\left(X ; \mathbf{I N}_{\bullet}\right) \cong H^{*}\left(X ; \mathcal{P}_{\bullet}\right)
$$

Moreover, if $X$ is compact and PL, one has also an isomorphism of algebras

$$
H^{*}\left(X ; \mathcal{P}_{\bullet}\right) \cong H_{n-*}^{\bar{t}-\bullet}\left(X ; \mathbb{F}_{2}\right),
$$

with the intersection product on the last term.

Proof The two first isomorphisms are consequences of the previous results on $\operatorname{cup}_{i}-$ products. The last one is established by Friedman in [6].

If we are interested only by the cup-product $\cup_{0}$, we may consider versions of the sheaves IN and $\mathcal{P}$ over any field. In this case, the previous corollary is still true for any field and not only for $\mathbb{F}_{2}$. With more work of this type, one also should be able to show the existence of an isomorphism between our definition of cup-product and the definition of Friedman and McClure [8].

\section{Pseudomanifolds with isolated singularities}

In this section, we determine Steenrod squares on the intersection cohomology of pseudomanifolds with isolated singularities. In this case, if the pseudomanifold is of dimension $n$, the perversity $\bar{p}$ is determined by one number, $\bar{p}(n)$. Recall now that the intersection cohomology of a cone $c Y$ on a space $Y$ is given by $H_{\mathrm{TW}, \bar{p}}^{r}(c Y)=H^{r}(Y)$, if $r \leq \bar{p}(n)$ and 0 otherwise. 
Proposition 5.1 Let $\bar{p}$ be a GM-perversity and $X$ an $n$-dimensional pseudomanifold obtained from a triangulated manifold with boundary $(W, \partial W)$ by attaching cones on the connected components $\left(\partial_{u} W\right)_{u \in I}$ of $\partial W$; ie $X$ is the pushout:

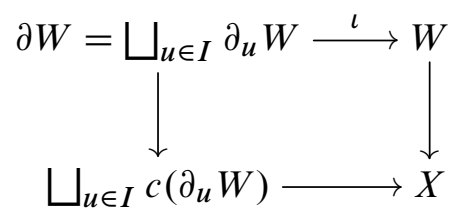

We filter the pseudomanifold $X$ by $\varnothing \subset\left\{\mathrm{v}_{u} \mid u \in I\right\} \subset X$, where $\mathrm{v}_{u}$ is the cone point of $c\left(\partial_{u} W\right)$. Then the following properties are satisfied:

(i) The cochain complex $\tilde{N}_{\bar{p}}^{*}(X)$ is quasi-isomorphic to the pullback in the category of cochain complexes $N^{*}(W) \oplus_{N^{*}(\partial W)} \tau_{\leq \bar{p}(n)} N^{*}(\partial W)$, where $\tau_{\leq \bar{p}(n)} N^{*}(\partial W)$ is the usual truncation (see $[2,1.10]$ ),

$$
\left(\tau_{\leq \bar{p}(n)} N^{*}(\partial W)\right)^{r}= \begin{cases}N^{r}(\partial W) & \text { if } r<\bar{p}(n), \\ \mathcal{Z} N^{\bar{p}(n)}(\partial W) & \text { if } r=\bar{p}(n), \\ 0 & \text { if } r>\bar{p}(n),\end{cases}
$$

in which $\mathcal{Z}$ denotes the vector space of cocycles. Moreover, the GM-perverse $\mathcal{E}(2)$-algebra $\bar{p} \mapsto \tilde{N}_{\bar{p}}^{*}(X)$ is quasi-isomorphic to the pullback in the category of

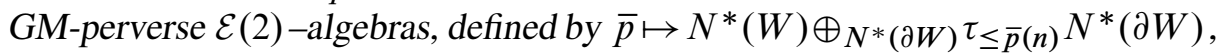
with the $\mathcal{E}(2)$-algebra structure on $N^{*}(-)$ defined in [1].

(ii) The intersection cohomology of $X$ is determined by

$$
H_{\mathrm{TW}, \bar{p}}^{k}(X)= \begin{cases}H^{k}(W) & \text { if } k \leq \bar{p}(n) \\ \operatorname{Ker}\left(H^{k}(W) \rightarrow H^{k}(\partial W)\right) & \text { if } k=\bar{p}(n)+1 \\ H^{k}(W, \partial W) & \text { if } k>\bar{p}(n)+1\end{cases}
$$

(iii) If $\left(\alpha, \iota^{*} \alpha\right) \in N^{*}(W) \oplus_{N^{*}(\partial W)} \tau_{\leq \bar{p}(n)} N^{*}(\partial W)$ is a cocycle of $\bar{p}$-intersection and $i$ is a positive integer, we have

$$
\mathrm{Sq}^{i}\left(\alpha, \iota^{*} \alpha\right)=\left(\mathrm{Sq}^{i} \alpha, \iota^{*} \mathrm{Sq}^{i} \alpha\right) \in H_{\mathrm{TW}, \mathcal{L}(\bar{p}, i)}^{*+i}(X) .
$$

Proof (i) Starting from a triangulation of $(W, \partial W)$, we may suppose that $X, W, \partial W$ and $\bigsqcup_{u \in I} c\left(\partial_{u} W\right)$ are triangulated in such a way that any simplex of the triangulation of $X$ is filtered, with filtration $\varnothing \subset\left\{\mathrm{v}_{u} \mid u \in I\right\} \subset X$.

Let $Y$ be one of the spaces above and $Y^{\tau}$ the associated triangulated space. In [7, Chapters 3 and 5], Friedman proves that the cochains $C_{\mathrm{GM}, \bar{p}}^{*}(Y)$ and $C_{\mathrm{GM}, \bar{p}}^{*}\left(Y^{\tau}\right)$ are quasi-isomorphic for any GM-perversity $\bar{p}$. Let $\bar{p}$ and $\bar{q}$ be two GM-perversities such that $\bar{p}(k)+\bar{q}(k)=k-2$. There exists a quasi-isomorphism between $C_{\mathrm{GM}, \bar{q}}^{*}(Y)$ and 
$\widetilde{C}_{\bar{p}}^{*}(Y)$; see [4, Theorem B] or Proposition 1.8. Recall also from Proposition 1.5 the existence of a quasi-isomorphism between $\widetilde{C}_{\bar{p}}^{*}(Y)$ and $\tilde{N}_{\bar{p}}^{*}(Y)$. Thus, the isomorphism

$$
C_{\mathrm{GM}, \bar{q}}^{*}\left(X^{\tau}\right) \cong C^{*}\left(W^{\tau}\right) \oplus_{\left(\oplus_{u \in I} C^{*}\left(\partial_{u} W^{\tau}\right)\right)}\left(\bigoplus_{u \in I} C_{\mathrm{GM}, \bar{q}}^{*}\left(c\left(\partial_{u} W\right)^{\tau}\right)\right),
$$

obtained by construction of the triangulations, gives quasi-isomorphisms

$$
\begin{aligned}
C_{\mathrm{GM}, \bar{q}}^{*}(X) & \simeq C^{*}(W) \oplus_{C^{*}(\partial W)}\left(\bigoplus_{u \in I} C_{\mathrm{GM}, \bar{q}}^{*}\left(c\left(\partial_{u} W\right)\right)\right) \\
& \simeq C^{*}(W) \oplus_{C^{*}(\partial W)}\left(\bigoplus_{u \in I} \tau_{\leq \bar{t}(n)-\bar{q}(n)} C^{*}\left(\partial_{u} W\right)\right) \\
& \simeq C^{*}(W) \oplus_{C^{*}(\partial W)} \tau_{\leq \bar{t}(n)-\bar{q}(n)} C^{*}(\partial W) \\
& \simeq N^{*}(W) \oplus_{N^{*}(\partial W)}{ }^{\tau_{\leq \bar{t}(n)-\bar{q}(n)}} N^{*}(\partial W) .
\end{aligned}
$$

Therefore, we have obtained a quasi-isomorphism

$$
\tilde{N}_{\bar{p}}^{*}(X) \simeq N^{*}(W) \oplus_{N^{*}(\partial W)} \tau_{\leq \bar{p}(n)} N^{*}(\partial W) .
$$

We now investigate the structure of the $\mathcal{E}(2)$-algebra. In [1], C Berger and B Fresse prove that a restriction map $N^{*}(Y) \rightarrow N^{*}(Z)$, induced by an inclusion $Z \hookrightarrow Y$, is a morphism of $\mathcal{E}(2)$-algebras. Therefore, we obtain functors from the lattice of GM-perversities (and $\bar{\infty}$ ) to GM-perverse $\mathcal{E}(2)$-algebras, defined by $\bar{p} \mapsto \tilde{N}_{\bar{p}}^{*}(X)$, $\bar{p} \mapsto N^{*}(W), \bar{p} \mapsto N^{*}(\partial W)$ and $\bar{p} \mapsto \tau_{\leq \bar{p}(n)} N^{*}(\partial W)$. Restriction maps define GMperverse $\mathcal{E}(2)$-algebra maps between $\tilde{N}_{\bar{p}}^{*}(X)$ and the three other GM-perverse $\mathcal{E}(2)$ algebras. From them, we obtain a GM-perverse $\mathcal{E}(2)$-algebra map

$$
\tilde{N}_{\bar{p}}^{*}(X) \rightarrow N^{*}(W) \oplus_{N^{*}(\partial W)} \tau_{\leq \bar{p}(n)} N^{*}(\partial W),
$$

whose codomain is a pullback in the category of GM-perverse $\mathcal{E}(2)$-algebras; see [1]. We have proved above that this last map is a quasi-isomorphism for each $\bar{p}$, and the first item of the statement is established.

(ii) An element of the previous sum is of the type $\left(\alpha, \iota^{*} \alpha\right)$, with $\iota^{*} \alpha$ of degree less than, or equal to, $\bar{p}(n)$. This means that, if $\alpha$ is of degree $k$, we must have

$$
\begin{cases}\iota^{*} \alpha=0 & \text { if } k>\bar{p}(n), \\ \iota^{*} \alpha \text { is a cocycle } & \text { if } k=\bar{p}(n) .\end{cases}
$$

This immediately implies that $H_{\mathrm{TW}, \bar{p}}^{k}(X)=H^{k}(W)$ if $k \leq \bar{p}(n)$ and also that $H_{\mathrm{TW}, \bar{p}}^{k}(X)=H^{k}(W, \partial W)$ if $k>\bar{p}(n)+1$. In degree $k=\bar{p}(n)+1$, the $\bar{p}$-intersection cohomology of $X$ is formed of the elements of $H^{k}(W)$ which are in the image of $H^{k}(W, \partial W)$, ie the kernel of $H^{k}(W) \rightarrow H^{k}(\partial W)$. 
(iii) The quasi-isomorphisms between $\tilde{N}_{\bar{p}}^{*}(X)$ and $N^{*}(W) \oplus_{N^{*}(\partial W)} \tau_{\leq \bar{p}(n)} N^{*}(\partial W)$ define a map of GM-perverse $\mathcal{E}(2)$-algebras, and therefore they are compatible with the cup $_{i}$-products. Also, the right-hand complex of (4) can be used for the determination of cup $_{i}$-products; ie we have

$$
\left(\alpha, \iota^{*} \alpha\right) \cup_{i}\left(\beta, \iota^{*} \beta\right)=\left(\alpha \cup_{i} \beta, \iota^{*} \alpha \cup_{i} \iota^{*} \beta\right),
$$

from which we deduce the announced formula for Steenrod squares.

Remark 5.2 We give a direct proof of the Goresky-Pardon conjecture in the case of isolated singularities. Let $\left(\alpha, \iota^{*} \alpha\right)$ be a cocycle in $N^{k}(W) \oplus_{N^{k}(\partial W)} \tau_{\leq \bar{p}(n)} N^{k}(\partial W)$. The perverse degree of the Steenrod square $\operatorname{Sq}^{j}\left(\alpha, \iota^{*} \alpha\right)=\left(\alpha, \iota^{*} \alpha\right) \cup_{k-j}\left(\alpha, \iota^{*} \alpha\right)$ satisfies the inequality

$$
\left\|\left(\alpha, \iota^{*} \alpha\right) \cup_{k-j}\left(\alpha, \iota^{*} \alpha\right)\right\| \leq_{(1)}\left|\iota^{*} \alpha \cup_{k-j} \iota^{*} \alpha\right| \leq_{(2)} k+j \leq_{(3)} \bar{p}(n)+j,
$$

where

- $\leq{ }_{(1)}$ comes from the fact that the perverse degree of a cochain is less than or equal to its usual degree,

- $\leq_{(2)}$ is a consequence of $\left|a \cup_{i} b\right| \leq|a|+|b|-i$,

- $\quad \leq_{(3)}$ uses $\iota^{*} \alpha=0$ if $k>\bar{p}(n)$.

Remark 5.3 The fact that the image of $H_{\mathrm{TW}, \bar{p}}^{*}(X)$ by $\mathrm{Sq}^{i}$ is in perversity $\mathcal{L}(\bar{p}, i)=$ $\min (2 \bar{p}, \bar{p}+i)$ is perfectly in phase with the characterization of the intersection cohomology of $X$, written in Proposition 5.1(ii). This remark follows from these observations for a cocycle $\left(\alpha, \iota^{*} \alpha\right) \in N^{k}(W) \oplus_{N^{k}(\partial W)} \tau_{\leq \bar{p}(n)} N^{k}(\partial W)$ :

- If $k \leq \bar{p}(n)$, then by definition of the Steenrod squares in $H^{*}(W)$, we have $\left|\mathrm{Sq}^{i}(\alpha)\right|=k+i \leq \bar{p}(n)+i$.

- If $i \leq \bar{p}(n)$, this implies $\left|\operatorname{Sq}^{i}\left(\alpha, \iota^{*} \alpha\right)\right| \leq \mathcal{L}(\bar{p}, i)(n)$.

- If $i>\bar{p}(n)$, we have $\mathrm{Sq}^{i}\left(\alpha, \iota^{*} \alpha\right)=\left(\mathrm{Sq}^{i} \alpha, \iota^{*} \mathrm{Sq}^{i} \alpha\right)=0$.

- If $k>\bar{p}(n)$, then $\iota^{*} \alpha=0$ and $\left|\mathrm{Sq}^{i}\left(\alpha, \iota^{*} \alpha\right)\right|=k+i>\bar{p}(n)+i \geq \mathcal{L}(\bar{p}, i)(n)$.

In conclusion, $\mathrm{Sq}^{i}$ respects the caesuras in the determination of the perverse cohomologies $H_{\mathrm{TW}, \bar{p}}^{*}(X)$ and $H_{\mathrm{TW}, \mathcal{L}(\bar{p}, i)}^{*}(X)$. Moreover, in degrees $k \leq \bar{p}(n)$, the Steenrod squares on $H_{\mathrm{TW}, \bar{p}}^{k}(X)$ coincide with the Steenrod squares on $H^{k}(W)$. 
Example 5.4 (Steenrod squares on the intersection cohomology of the suspension of a manifold) Let $X$ be an (n-1)-dimensional manifold and $\bar{p}$ a GM-perversity. The following pushout defines $\Sigma X$ as in Proposition 5.1:

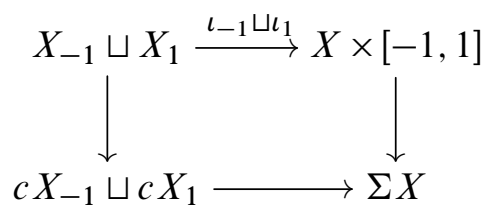

where $\iota_{1}: X_{1}=X \times\{1\} \rightarrow X \times[-1,1]$ and $\iota_{-1}: X_{-1}=X \times\{-1\} \rightarrow X \times[-1,1]$ are the canonical injections. From (i) of Proposition 5.1, we know that $\tilde{N}_{\bar{p}}^{*}(\Sigma X)$ is quasi-isomorphic to the cochain complex

$$
\begin{array}{r}
N^{*}(X \times[-1,1])^{<\bar{p}(n)} \oplus\left\{\alpha \in N^{\bar{p}(n)}(X \times[-1,1]) \mid d \iota_{1}^{*}(\alpha)=d \iota_{-1}^{*}(\alpha)=0\right\} \\
\oplus\left(\operatorname{Ker} \iota_{1}^{*} \cap \operatorname{Ker} \iota_{-1}^{*}\right)^{>\bar{p}(n)},
\end{array}
$$

in which the superscript refers to the degree. For instance, $(A)^{<k}$ is the set of elements of $A$ of degree less than $k$.

The suspension $\Sigma X$ can also be obtained as a cofiber, $X_{1} \sqcup X_{-1} \rightarrow X \times[-1,1] \rightarrow \Sigma X$, which gives a short exact sequence

$$
0 \rightarrow\left(\operatorname{Ker} \iota_{1}^{*} \cap \operatorname{Ker} \iota_{-1}^{*}\right) \hookrightarrow N^{*}(X \times[-1,1]) \stackrel{\left(\iota_{1}^{*}, \iota_{-1}^{*}\right)}{\longrightarrow} N^{*}\left(X_{1}\right) \oplus N^{*}\left(X_{-1}\right) \rightarrow 0 .
$$

The morphism of $\mathcal{E}(2)$-algebras [1], $N^{*}(\Sigma X) \rightarrow N^{*}(X \times[-1,1])$, lifts as a quasiisomorphism of cochain complexes, $N^{*}(\Sigma X) \rightarrow\left(\operatorname{Ker} \iota_{1}^{*} \cap \operatorname{Ker} \iota_{-1}^{*}\right)$. From (5) and the previous observation, we deduce the intersection cohomology of the suspension $\Sigma X$ as

$$
H_{\mathrm{TW}, \bar{p}}^{k}(\Sigma X)= \begin{cases}H^{k}(X) & \text { if } k \leq \bar{p}(n), \\ 0 & \text { if } k=\bar{p}(n)+1, \\ H^{k}(\Sigma X)=H^{k-1}(X) & \text { if } k>\bar{p}(n)+1 .\end{cases}
$$

With Remark 5.3, we know that, in degrees $k \leq \bar{p}(n)$, the Steenrod squares on $H_{\mathrm{TW}, \bar{p}}^{k}(\Sigma X)$ coincide with the Steenrod squares on $H^{k}(X)$. Moreover, the intersection of kernels being endowed with the induced structure of an $\mathcal{E}(2)$-algebra of $N^{*}(X \times[-1,1])$, the quasi-isomorphism $N^{*}(\Sigma X) \rightarrow\left(\operatorname{Ker} \iota_{1}^{*} \cap \operatorname{Ker} \iota_{-1}^{*}\right)$ is a morphism of $\mathcal{E}(2)$-algebras; see [1]. Thus, in degrees $k>\bar{p}(n)+1$, the Steenrod squares on $H_{\mathrm{TW}, \bar{p}}^{k}(\Sigma X)$ coincide with the Steenrod squares on $H^{k}(\Sigma X)$, which are the suspensions of the Steenrod squares on $X$.

We consider now the case of the Thom space of a vector bundle, $\mathbb{R}^{m} \rightarrow E \rightarrow B$. 
Example 5.5 (Steenrod squares on the intersection cohomology of a Thom space) Let $\mathbb{R}^{m} \rightarrow D_{E} \stackrel{g}{\rightarrow} B$ be the disk-bundle of associated sphere-bundle $S^{m-1} \rightarrow S_{E} \stackrel{f}{\rightarrow} B$. The Thom space $\mathrm{Th}(E)$ is built from the disk-bundle along the process described in Proposition 5.1. We filter $\operatorname{Th}(E)$ by the point of compactification. Let $\bar{p}$ be a GM-perversity entirely determined in this case by the number $\bar{p}(n)$ with $n=\operatorname{dim} E$. In this example, we prove that the Steenrod squares on $H_{\mathrm{TW}, \bar{p}}^{*}(\mathrm{Th}(E))$ are entirely determined by the Steenrod squares on the base space and the Stiefel-Whitney classes of the bundle.

Denote by $c \in H^{m}(B)$ the Euler class and by $\theta \in H^{m}(\operatorname{Th}(E))$ the Thom class. Let $j: D_{E} \rightarrow \mathrm{Th}(E)$ be the canonical map, and recall that the Thom isomorphism $\mathcal{T} h: H^{k-m}(B) \rightarrow H^{k}(\mathrm{Th}(E)) \cong H^{k}\left(D_{E}, S_{E}\right)$ is defined by $\mathcal{T} h(\gamma)=g^{*}(\gamma) \cup \theta$. The Euler and the Thom classes are connected by the two exact sequences

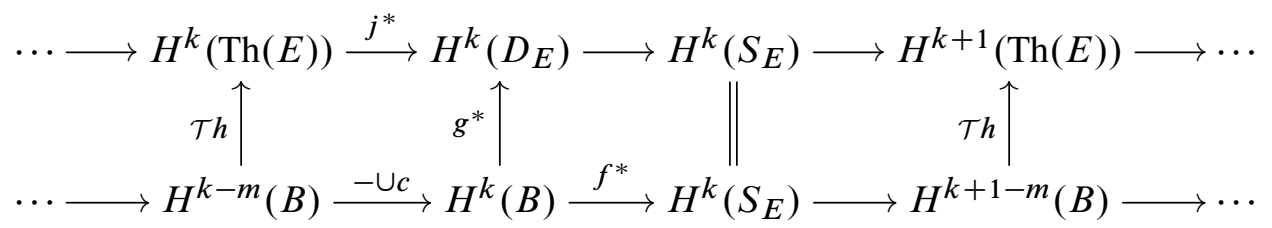

and the fact that $j^{*}(\theta)=g^{*}(c)$. From Proposition 5.1, we know that the complex $\tilde{N}_{\bar{p}}^{*}(\mathrm{Th}(E))$ is quasi-isomorphic to

$$
\begin{aligned}
\mathcal{N}^{*}=N^{*}(B) \oplus_{N^{*}\left(S_{E}\right)} \tau_{\leq \bar{p}(n)} N^{*}\left(S_{E}\right) \\
\cong N^{<\bar{p}(n)}(B) \oplus\left\{\alpha \in N^{\bar{p}(n)}(B) \mid d f^{*}(\alpha)=0\right\} \\
\oplus \operatorname{Ker}\left(N^{k}(B) \stackrel{f^{*}}{\longrightarrow} N^{k}\left(S_{E}\right)\right)^{>\bar{p}(n)} .
\end{aligned}
$$

Thus we recover (see [16, page 77]) the intersection cohomology of the Thom space,

$$
H_{\mathrm{TW}, \bar{p}}^{k}(\mathrm{Th}(E))=H^{k}(\mathcal{N})= \begin{cases}H^{k}(B) & \text { if } k \leq \bar{p}(n) \\ (\operatorname{Im}(-\cup c))^{k} & \text { if } k=\bar{p}(n)+1 \\ H^{k-m}(B) \cong \mathcal{T} h H^{k}(\operatorname{Th}(E)) & \text { if } k>\bar{p}(n)+1\end{cases}
$$

- If $k \leq \bar{p}(n)+1$, the Steenrod squares $\mathrm{Sq}^{i}: H_{\bar{p}}^{k}(\mathrm{Th}(E)) \rightarrow H_{\mathcal{L}(\bar{p}, i)}^{k+i}(\mathrm{Th}(E))$ coincide with the Steenrod squares $\mathrm{Sq}^{i}: H^{k}(B) \rightarrow H^{k+i}(B)$; see Remark 5.3.

- Let $k>\bar{p}(n)+1$ and $\gamma \in H^{k-m}(B)$. The (classical) internal Cartan formula gives $\operatorname{Sq}^{j}\left(g^{*}(\gamma) \cup \theta\right)=\sum_{\ell=0}^{j} \operatorname{Sq}^{j-\ell}\left(g^{*}(\gamma)\right) \cup \operatorname{Sq}^{\ell}(\theta)=\sum_{\ell=0}^{j} g^{*}\left(\operatorname{Sq}^{j-\ell}(\gamma)\right) \cup g^{*}\left(\omega_{\ell}\right) \cup \theta$,

where the $\omega_{\ell}$ are the Stiefel-Whitney classes of the fibration $f$; see [19, page 91]. Set $\mu=g^{*}(\gamma) \cup \theta=\mathcal{T} h(\gamma) \in H^{k}(\mathrm{Th}(E))$. In this range of degrees, the Steenrod 
squares on $\mathrm{Th}(E)$, denoted by $\mathrm{Sq}_{\mathrm{Th}}$, and the Steenrod squares on $B$, denoted by $\mathrm{Sq}_{B}$, are related by

$$
\mathrm{Sq}_{\mathrm{Th}}^{j}(\mu)=\sum_{\ell=0}^{j} g^{*}\left(\mathrm{Sq}_{B}^{j-\ell}(\gamma) \cup \omega_{\ell}\right) \cup \theta .
$$

With the Thom isomorphism $\mathcal{T} h: H^{k-m}(B) \rightarrow H^{k}(\mathrm{Th}(E))$ the previous formula can be written as

$$
\mathrm{Sq}_{\mathrm{Th}}^{j}(\mu)=\mathcal{T} h\left(\sum_{\ell=0}^{j} \mathrm{Sq}_{B}^{j-\ell}\left(\mathcal{T} h^{-1}(\mu)\right) \cup \omega_{\ell}\right) .
$$

\section{Example of a fibration with fiber a cone}

In this section, we construct an example showing the interest of the lifting of the image of $\mathrm{Sq}^{i}$ to the perversity $\mathcal{L}(\bar{p}, i)$ instead of $2 \bar{p}$. As the case of $\mathrm{Sq}^{1}$ was analyzed in [12], we choose an example with $\mathrm{Sq}^{2}$.

Proposition 6.1 There exists a pseudomanifold $X$ and a GM-perversity $\bar{p}$ with an explicit nontrivial perverse square

$$
0 \neq \mathrm{Sq}^{2}: H_{\mathrm{TW}, \bar{p}}^{6}(X) \rightarrow H_{\mathrm{TW}, \mathcal{L}(\bar{p}, 2)}^{8}(X),
$$

whose composition with the canonical map $H_{\mathrm{TW}, \mathcal{L}(\bar{p}, 2)}^{8}(X) \rightarrow H_{\mathrm{TW}, 2 \bar{p}}^{8}(X)$ is zero.

Proof To begin with, we describe the general strategy of the proof. The first step is the construction of a fibration $S^{7} \times S^{4} \rightarrow E \stackrel{\varphi}{\rightarrow} \mathbb{C} P(2)$, with a nontrivial differential on a generator $a_{7}$ of $H^{7}\left(S^{7} \times S^{4}\right)$, in the Serre spectral sequence. Secondly, we consider the fiberwise conification $c\left(S^{7} \times S^{4}\right) \rightarrow X \stackrel{\psi}{\longrightarrow} \mathbb{C} P(2)$ of the fibration $\varphi$. The space $X$ is a pseudomanifold. A GM-perversity $\bar{p}$ on $X$ is determined by the value $\bar{p}(12)=k$, and we denote it by $\bar{k}$. (As $\bar{p}$ is a GM-perversity, we have $k \leq 10$.) In our fibration, depending on the value of $k$, the element $a_{7}$ is a class of $\bar{p}$-intersection or it is not; more precisely, we get $H_{\mathrm{TW}, \bar{k}}^{8}(X) \neq 0$ if $k=6$ and $H_{\mathrm{TW}, \bar{k}}^{8}(X)=0$ if $k=8$. This property generates a nontrivial Steenrod square $\mathrm{Sq}^{2}: H_{\mathrm{TW}, \bar{p}}^{6}(X) \rightarrow H_{\mathrm{TW}, \mathcal{L}(\bar{p}, 2)}^{8}(X)$ such that the composite with the canonical map

$$
H_{\mathrm{TW}, \bar{p}}^{6}(X) \stackrel{\mathrm{Sq}^{2}}{\longrightarrow} H_{\mathrm{TW}, \mathcal{L}(\bar{p}, 2)}^{8}(X) \rightarrow H_{\mathrm{TW}, 2 \bar{p}}^{8}(X)
$$

is the zero map. Details are as follows.

- First, we observe, from the cellular approximation theorem and the construction of $K(\mathbb{Z}, 8)$, that the classifying map of the top class $\mathbb{C} P(2) \times S^{4} \rightarrow K(\mathbb{Z}, 8)$ lifts as 
a map $f: \mathbb{C P}(2) \times S^{4} \rightarrow S^{8}$. We denote by $p_{1}: E \rightarrow \mathbb{C P}(2) \times S^{4}$ the pullback of the Hopf fibration $S^{15} \rightarrow S^{8}$ along $f$. We compose $p_{1}$ with the trivial fibration $p_{2}: \mathbb{C} P(2) \times S^{4} \rightarrow \mathbb{C} P(2)$ and obtain a fibration

$$
\varphi: E \rightarrow \mathbb{C} P(2),
$$

whose fiber $F$ is $S^{7} \times S^{4}$. To show this last point, consider the commutative diagram:

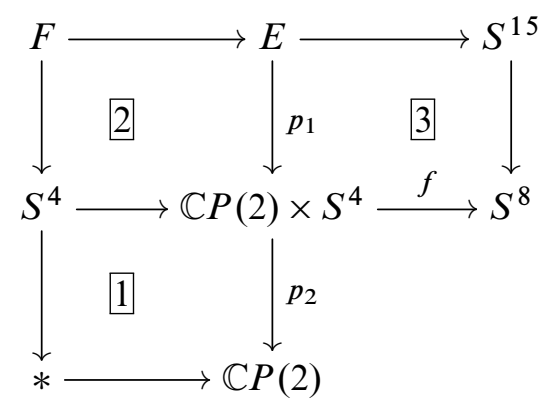

The rectangle formed of 1 and 2 is a pullback. As 1 is a pullback, we deduce [17, Section III.4] that 2 is a pullback. Therefore, the rectangle formed of 2 and 3 is a pullback, and the triviality of the map $S^{4} \rightarrow S^{8}$ implies that $F$ is $S^{7} \times S^{4}$.

We study now the Serre spectral sequence of the fibration $\varphi$. We denote by $a_{4}, a_{7}$ and $a_{7} \times a_{4}$ the generators of the reduced cohomology of $S^{7} \times S^{4}$, and by $x$ and $x^{2}$ the generators of the reduced cohomology of $\mathbb{C} P(2)$. An inspection of the degrees in the differentials $d_{r}: E_{r}^{s, t} \rightarrow E_{r}^{s+r, t-r+1}$ shows that the only differential which can be potentially nontrivial is

$$
d_{4}: E_{4}^{0,7}=E_{2}^{0,7}=\mathbb{F}_{2} a_{7} \rightarrow E_{4}^{4,4}=E_{2}^{4,4}=\mathbb{F}_{2}\left(x^{2} \otimes a_{4}\right) .
$$

By definition of $S^{7} \rightarrow E \rightarrow \mathbb{C} P(2) \times S^{4}$ as a pullback of the Hopf fibration, we already know [21, Section III.4] that the top class $a_{7}$ of $S^{7}$ transgresses on the product $x^{2} \times a_{4}$. This gives $d_{4}\left(a_{7}\right)=x^{2} \otimes a_{4}$ in the Serre spectral sequence of the fibration $\varphi: E \rightarrow \mathbb{C} P(2)$.

We continue with the determination of the image of the cohomology class $x \otimes a_{4}$ by $\mathrm{Sq}^{2}$ in $H^{*}(\mathbb{C} P(2)) \otimes H^{*}\left(S^{7} \times S^{4}\right)$. From the external Cartan formula, we have

$$
\mathrm{Sq}^{2}\left(x \otimes a_{4}\right)=\mathrm{Sq}^{2}(x) \otimes a_{4}+\mathrm{Sq}^{1}(x) \otimes \mathrm{Sq}^{1}\left(a_{4}\right)+x \otimes \mathrm{Sq}^{2}\left(a_{4}\right) \text {. }
$$

The last two terms are zero for degree reasons. The equality $\operatorname{Sq}^{2}(x)=x^{2}$ gives

$$
\mathrm{Sq}^{2}\left(x \otimes a_{4}\right)=x^{2} \otimes a_{4} .
$$


- The second step is the fiberwise conification $c\left(S^{7} \times S^{4}\right) \rightarrow X \stackrel{\psi}{\longrightarrow} \mathbb{C} P(2)$ of the fibration $\varphi$. If $x \in \mathbb{C} P(2)$, we denote by $\left(S^{7} \times S^{4}\right)_{x}$ the fiber over $x$ and by $\mathrm{v}_{x}$ the cone point of the cone $c\left(\left(S^{7} \times S^{4}\right)_{x}\right)$. A continuous section $\mu$ of $\psi$, defined by $\mu(x)=\mathrm{v}_{x}$, identifies $\mathbb{C} P^{2}$ to a closed subspace of $X$. We filter $X$ by $\varnothing \subset X_{0}=\mathbb{C} P(2) \subset X$. Observe that the singular set in $X$ is $\mathbb{C} P(2)$ and that the link of a singular point is $S^{7} \times S^{4}$.

Let $\bar{k}$ be a GM-perversity. The intersection cohomology $H_{\mathrm{TW}, \bar{k}}^{*}(X)$ is the abutment (see [5, Theorem 3.5]) of a Serre spectral sequence with

$$
\bar{k}_{2}^{r, s}=H^{r}(\mathbb{C} P(2)) \otimes H_{\mathrm{TW}, \bar{k}}^{s}\left(c\left(S^{7} \times S^{4}\right)\right) .
$$

We may replace the right-hand term of this tensor product by its value and obtain

$$
\bar{k} E_{2}^{r, s}=H^{r}(\mathbb{C} P(2)) \otimes H^{s}\left(S^{7} \times S^{4}\right)
$$

if $s \leq k$ and 0 otherwise. The existence of a morphism $E \rightarrow X$ over the identity on $\mathbb{C} P(2)$ gives a morphism of spectral sequences, $\left(\bar{k}_{*}^{r, s}, d_{*}\right) \rightarrow\left(E_{*}^{r, s}, d_{*}\right)$. From our previous determination of the Serre spectral sequence $\left(E_{*}^{r, s}, d_{*}\right)$ associated to the fibration $\varphi: E \rightarrow \mathbb{C P}(2)$, we deduce that the differentials $d_{*}$ of $\bar{k}_{*} E_{*}^{r, s}$ are zero, except $d_{4}\left(a_{7}\right)=x^{2} \otimes a_{4}$ if $7 \leq k$. Thus, in perversity $k<7$, as the class $a_{7}$ is not of $\bar{k}$-intersection, the class $x^{2} \otimes a_{4}$ survives and $H_{\mathrm{TW}, \bar{k}}^{8}(X) \neq 0$. But if $k=8$, the class $a_{7}$ is of $\overline{8}$-intersection and kills the element $x^{2} \otimes a_{4}$ (which is the only element of degree 8 in the $E_{2}$-term). Thus $H_{\mathrm{TW}, \overline{8}}^{8}(X)=0$.

The square $\mathrm{Sq}^{2}$, that we have previously determined, arises in the GM-perversity $\overline{4}$, and we have

$$
\mathrm{Sq}^{2}: H_{\mathrm{TW}, \overline{4}}^{6}(X)=\mathbb{F}_{2}\left(x \otimes a_{4}\right) \rightarrow H_{\mathrm{TW}, \overline{4}}^{8}(X)=\mathbb{F}_{2}\left(x^{2} \otimes a_{4}\right) .
$$

Observe that $\overline{6}=\mathcal{L}(\overline{4}, \overline{4}+2)$ is a GM-perversity and thus, with the argument above, $\mathrm{Sq}^{2}$ still survives as map from $H_{\mathrm{TW}, \overline{4}}^{6}$ to $H_{\mathrm{TW}, \overline{6}}^{8}=H_{\mathrm{TW}, \overline{4}}^{8}$. But, for the GM-perversity $\overline{8}=2 \times \overline{4}$, as $H_{\mathrm{TW}, \overline{8}}^{8}(X)=0$, this square $\mathrm{Sq}^{2}$ disappears if we express it as a map from $H_{\mathrm{TW}, \overline{4}}^{6}$ to $H_{\mathrm{TW}, 2 \times \overline{4}}^{8}$.

\section{Topological invariance of the Steenrod squares in intersection cohomology}

In the case of PL-pseudomanifolds, we know from [13] that the Steenrod squares are topological invariants, as homomorphisms $H_{\mathrm{TW}, \bar{p}}^{r}(X) \rightarrow H_{\mathrm{TW}, 2 \bar{p}}^{r+i}(X)$. In this section, we prove that the lifting we have introduced before, $\mathrm{Sq}^{i}: H_{\mathrm{TW}, \bar{p}}^{r}(X) \rightarrow H_{\mathrm{TW}, \mathcal{L}(\bar{p}, i)}^{r+i}(X)$, is also a topological invariant. The proof is based on the original combinatorial description of Steenrod squares made in [22]. 
Theorem D Let $X$ be an $n$-dimensional PL-pseudomanifold and $\bar{p}$ a GM-perversity. Then the Steenrod squares $\mathrm{Sq}^{i}: H_{\mathrm{TW}, \bar{p}}^{*}(X) \rightarrow H_{\mathrm{TW}, \mathcal{L}(\bar{p}, i)}^{*+i}(X)$ do not depend on the stratification of $X$.

Theorem $\mathrm{D}$ is a direct consequence of Proposition 7.1 and Proposition 7.9. Before stating and proving these two results, we need to introduce some material. First, recall from [15, page 150] and [7, Chapter 2] the existence of a PL-pseudomanifold $X^{*}$ which is an intrinsic coarsest stratification of $X$, together with a stratified map $v: X \rightarrow X^{*}$ defined by the identity map; see [4, Definition A.18]. In [15], H King proves that $v$ induces a quasi-isomorphism between the Goresky-MacPherson chain (and cochain) complexes. Here we consider the map $\chi$, induced by $v$, between the Thom-Whitney complexes.

Proposition 7.1 Let $X$ be an $n$-dimensional PL-pseudomanifold, and let $\bar{p}$ be a GM-perversity. Then the canonical map $v: X \rightarrow X^{*}$ induces a quasi-isomorphism $\chi: \tilde{N}_{\bar{p}}^{*}\left(X^{*}\right) \rightarrow \tilde{N}_{\bar{p}}^{*}(X)$.

\section{Construction of $\chi$, the local step}

Before giving the proof, we detail the construction of $\chi$ based on the effect of $\nu: X \rightarrow X^{*}$ on filtered simplices of $X$. Let $\sigma: \Delta=\Delta^{j_{0}} * \cdots * \Delta^{j_{n}} \rightarrow X$ be a filtered simplex of $X$. Suppose that

- for some integer $0 \leq i \leq n-1$, the set $\sigma\left(\Delta^{j_{0}} * \cdots * \Delta^{j_{i}}\right) \backslash \sigma\left(\Delta^{j_{0}} * \cdots * \Delta^{j_{i-1}}\right)$ is included in an $i$-stratum of $X$ which "disappears" inside an $(i+1)$-stratum of $X^{*}$,

- for the other indices $\ell \neq i$, the corresponding strata of $\sigma(\Delta)$ stay unmodified.

Then the filtered simplex $\sigma: \Delta=\Delta^{j_{0}} * \cdots * \Delta^{j_{n}} \rightarrow X$ becomes a filtered simplex of $X^{*}$, namely $v \circ \sigma: \Delta(i)=\Delta^{k_{0}} * \cdots * \Delta^{k_{n}} \rightarrow X^{*}$, with

$$
\left\{\begin{array}{l}
k_{\ell}=j_{\ell} \quad \text { if } \quad \ell<i \text { or } \ell>i+1 \\
k_{i}=-1 \quad \text { and } \quad k_{i+1}=j_{i}+j_{i+1}+1 .
\end{array}\right.
$$

This process is called an elementary amalgamation. The simplex $v \circ \sigma: \Delta \rightarrow X^{*}$ can, in general, be written as a filtered simplex after a finite number of elementary amalgamations. As we work with blow-ups, we need to consider two cases, depending if $i+1=n$ or not. We write

$$
\tilde{N}^{*}(\Delta)=N^{*}\left(c \Delta^{j_{0}}\right) \otimes \cdots \otimes N^{*}\left(c \Delta^{j_{i}}\right) \otimes N^{*}\left(c \Delta^{j_{i+1}}\right) \otimes \cdots \otimes N^{*}\left(\Delta^{j_{n}}\right)
$$


and

$$
\tilde{N}^{*}(\Delta(i))=\left\{\begin{aligned}
N^{*}\left(c \Delta^{j_{0}}\right) & \otimes \cdots \otimes N^{*}(c \varnothing) & \\
& \otimes N^{*}\left(c \Delta^{j_{i}+j_{i+1}+1}\right) \otimes \cdots \otimes N^{*}\left(\Delta^{j_{n}}\right) & \text { if } i \neq n-1, \\
N^{*}\left(c \Delta^{j_{0}}\right) & \otimes \cdots \otimes N^{*}(c \varnothing) \otimes N^{*}\left(\Delta^{j_{n-1}+j_{n}+1}\right) & \text { if } i=n-1 .
\end{aligned}\right.
$$

We define below two morphisms,

$$
\alpha: N^{*}\left(c \Delta^{a+b+1}\right) \rightarrow N^{*}\left(c \Delta^{a}\right) \otimes N^{*}\left(c \Delta^{b}\right)
$$

and

$$
\beta: N^{*}\left(c \Delta^{a+b+1}\right) \rightarrow N^{*}\left(c \Delta^{a}\right) \otimes N^{*}\left(\Delta^{b}\right),
$$

which correspond to the cases $i \neq n-1$ and $i=n-1$.

Let $\mathrm{v}$ be the cone point of $c \varnothing$. We use $\alpha$ and $\beta$ for the definition of a morphism $\xi_{i}: \tilde{N}^{*}(\Delta(i)) \rightarrow \tilde{N}^{*}(\Delta)$ as follows. If $\Phi=\sum_{j} \Phi_{0, j} \otimes \cdots \otimes \Phi_{n, j} \in \tilde{N}^{*}(\Delta(i))$, then for $i \neq n-1$, we set

(7) $\xi_{i}(\Phi)=\sum_{j} \Phi_{i, j}([\mathrm{v}]) \cdot \Phi_{0, j} \otimes \cdots \otimes \Phi_{i-1, j} \otimes \alpha\left(\Phi_{i+1, j}\right) \otimes \Phi_{i+2, j} \otimes \cdots \otimes \Phi_{n, j}$, and for $i=n-1$,

$$
\xi_{i}(\Phi)=\sum_{j} \Phi_{n-1, j}([\mathrm{v}]) \cdot \Phi_{0, j} \otimes \cdots \otimes \Phi_{n-2, j} \otimes \beta\left(\Phi_{n, j}\right) .
$$

These $\xi_{i}$ are the local ingredients used in the (global) definition of $\chi$, stated below.

\section{Construction of $\alpha: N^{*}\left(c \Delta^{a+b+1}\right) \rightarrow N^{*}\left(c \Delta^{a}\right) \otimes N^{*}\left(c \Delta^{b}\right)$}

We define $\alpha$ by its values on the elements of a basis. If $L$ is one of the simplicial complexes, $c \Delta^{a}, c \Delta^{b}$ or $c \Delta^{a+b+1}$, we denote by $\left\{1_{F}\right\}$ the dual basis of $N^{*}(L)$ obtained from the basis of faces $F$ of $L$.

If we represent by $F_{a}$ the faces of $\Delta^{a}$ and by $F_{b}$ the faces of $\Delta^{b}$, a face of $c \Delta^{a+b+1}$ is of the type $c\left(F_{a} * F_{b}\right)$ or $F_{a} * F_{b}$, where $F_{a}$ and $F_{b}$ can also be the empty set. A linear map $\alpha$ is entirely determined by

$$
\begin{cases}\alpha\left(1_{c\left(F_{a} * F_{b}\right)}\right)=1_{c F_{a}} \otimes 1_{c F_{b}} & \text { the cases } F_{a}=\varnothing \text { and } F_{b}=\varnothing \text { being included, } \\ \alpha\left(1_{F_{a} * F_{b}}\right)=1_{c F_{a}} \otimes 1_{F_{b}} & \text { if } F_{b} \neq \varnothing, \text { the case } F_{a}=\varnothing \text { being included, } \\ \alpha\left(1_{F_{a}}\right)=1_{F_{a}} \otimes 1_{\mathrm{v}_{b}}+1_{F_{a}} \otimes 1_{\mathbb{V}_{b}}, & \end{cases}
$$

where $1_{\mathbb{V}_{b}}$ is the sum of $1_{\mathrm{p}}$ when $\mathrm{p}$ runs in the set of vertices of $\Delta^{b}$ and $\mathrm{v}_{b}$ is the cone point of $c \Delta^{b}$. 


\section{Construction of $\beta: N^{*}\left(\Delta^{a+b+1}\right) \rightarrow N^{*}\left(c \Delta^{a}\right) \otimes N^{*}\left(\Delta^{b}\right)$}

With the previous notation, the linear map $\beta$ is defined by

$$
\left\{\begin{array}{l}
\beta\left(1_{F_{a} * F_{b}}\right)=1_{c F_{a}} \otimes 1_{F_{b}} \quad \text { if } F_{b} \neq \varnothing, \text { the case } F_{a}=\varnothing \text { being included, } \\
\beta\left(1_{F_{a}}\right)=1_{F_{a}} \otimes 1_{\mathbb{V}_{b}} .
\end{array}\right.
$$

These maps satisfy the next properties whose proofs are postponed until after the proof of Proposition 7.1.

Lemma 7.2 The two morphisms, $\alpha: N^{*}\left(c \Delta^{a+b+1}\right) \rightarrow N^{*}\left(c \Delta^{a}\right) \otimes N^{*}\left(c \Delta^{b}\right)$ and $\beta: N^{*}\left(\Delta^{a+b+1}\right) \rightarrow N^{*}\left(c \Delta^{a}\right) \otimes N^{*}\left(\Delta^{b}\right)$, are compatible with the differentials and the restrictions to faces of $\Delta^{a}$ and $\Delta^{b}$.

Lemma 7.3 The morphism $\xi_{i}: \tilde{N}^{*}(\Delta(i)) \rightarrow \tilde{N}^{*}(\Delta)$ is compatible with the differentials and the restrictions to faces of the $\Delta^{j_{\ell}}$. Moreover, it respects the perverse degree, ie $\xi_{i}\left(\tilde{N}_{\bar{p}}^{*}(\Delta(i))\right) \subset \tilde{N}_{\bar{p}}^{*}(\Delta)$ for any GM-perversity $\bar{p}$.

\section{Construction of $\chi: \tilde{N}^{*}\left(X^{*}\right) \rightarrow \tilde{N}^{*}(X)$, the global step}

Let $\sigma: \Delta_{\sigma}=\Delta^{j_{0}} * \cdots * \Delta^{j_{n}} \rightarrow X$ be a filtered simplex of $X$, with blow-up $\tilde{\Delta}_{\sigma}=$ $c \Delta^{j_{0}} \times \cdots \times \Delta^{j_{n}}$. As we have noted before, the domain of the filtered simplex $\nu \circ \sigma: \Delta_{\nu \circ \sigma}=\Delta^{k_{0}} * \cdots * \Delta^{k_{n}} \rightarrow X^{*}$ has a different decomposition, obtained by a succession of elementary amalgamations. We denote by $\widetilde{\Delta}_{\nu \circ \sigma}$ the associated blow-up.

These elementary amalgamations give a finite sequence of decompositions $\Delta\left(i_{\ell}\right)_{0 \leq \ell \leq m}$ such that $\Delta(0)=\Delta^{j_{0}} * \cdots * \Delta^{j_{n}}=\Delta_{\sigma}$ and $\Delta(m)=\Delta^{k_{0}} * \cdots * \Delta^{k_{n}}=\Delta_{\nu \circ \sigma}$. Two consecutive terms correspond to an elementary amalgamation, ie $\Delta\left(i_{\ell}\right)=\Delta^{x_{0}} * \cdots * \Delta^{x_{n}}$ and $\Delta\left(i_{\ell+1}\right)=\Delta^{y_{0}} * \cdots * \Delta^{y_{n}}$, with

$$
\left\{\begin{array}{l}
y_{u}=x_{u} \quad \text { if } \quad u<i_{\ell} \text { or } u>i_{\ell}+1 \\
y_{i_{\ell}}=-1 \quad \text { and } \quad y_{i_{\ell}+1}=x_{i_{\ell}}+x_{i_{\ell+1}}+1
\end{array}\right.
$$

Recall the map $\xi_{i_{\ell}}: \tilde{N}^{*}\left(\Delta\left(i_{\ell+1}\right)\right) \rightarrow \tilde{N}^{*}\left(\Delta\left(i_{\ell}\right)\right)$ defined in (7) and (8). We set

$$
\chi_{\sigma}=\xi_{i_{0}} \circ \cdots \circ \xi_{i_{m-1}} .
$$

Finally, by Lemma 7.2, we have a map $\chi: \tilde{N}^{*}\left(X^{*}\right) \rightarrow \tilde{N}^{*}(X)$, defined on $\sigma: \Delta \rightarrow X$ and $\Phi \in \tilde{N}^{*}\left(X^{*}\right)$, by

$$
\chi(\Phi)_{\sigma}=\chi_{\sigma}\left(\Phi_{\nu \circ \sigma}\right)
$$


Proof of Proposition 7.1 By Lemma 7.3, the previous map $\chi: \tilde{N}^{*}\left(X^{*}\right) \rightarrow \tilde{N}^{*}(X)$ is a cochain map which restricts as $\chi: \tilde{N}_{\bar{p}}^{*}\left(X^{*}\right) \rightarrow \tilde{N}_{\bar{p}}^{*}(X)$.

There also exists a map eval $N: \tilde{N}^{*}(X) \rightarrow \operatorname{hom}\left(N_{*}^{\mathrm{GM}}(X), \mathbb{F}_{2}\right)$ defined as follows. For any $\Phi \in \tilde{N}^{*}(X)$ and $\sigma: \Delta^{j_{0}} * \cdots * \Delta^{j_{n}} \rightarrow X$, with $\Phi_{\sigma}=\sum_{j} \Phi_{0, j} \otimes \cdots \otimes \Phi_{n, j} \in$ $N^{*}\left(c \Delta^{j_{0}}\right) \otimes \cdots \otimes N^{*}\left(\Delta^{j_{n}}\right)$, we set

$$
\operatorname{eval}_{N}(\Phi)(\sigma)=\sum_{j} \Phi_{0, j}\left(\left[c \Delta^{j_{0}}\right]\right) \cdots \Phi_{n, j}\left(\left[\Delta^{j_{n}}\right]\right) .
$$

Let $\bar{q}$ be the GM-perversity such that $\bar{p}+\bar{q}=\bar{t}$. We have that the canonical morphism $\rho_{*}: C_{*}(-) \rightarrow N_{*}(-)$ induces $\rho^{*}: \operatorname{hom}\left(N_{*}^{\mathrm{GM}, \bar{q}}(-), \mathbb{F}_{2}\right) \rightarrow \operatorname{hom}\left(C_{*}^{\mathrm{GM}, \bar{q}}(-), \mathbb{F}_{2}\right)$ and $\tilde{\rho}: \tilde{N}_{\bar{p}}^{*}(-) \rightarrow \tilde{C}_{\bar{p}}^{*}(-)$. The previous map eval $N$ is connected with the morphism eval, introduced in the proof of Lemma 4.2, by $\rho^{*} \circ$ eval $_{N}=$ eval $\circ \tilde{\rho}$. As $\tilde{\rho}$ and eval are quasi-isomorphisms (see Proposition 1.5 and Proposition 1.8), we know that the composite $\rho^{*} \circ \operatorname{eval}_{N}$ is a quasi-isomorphism. Now consider the following diagram:

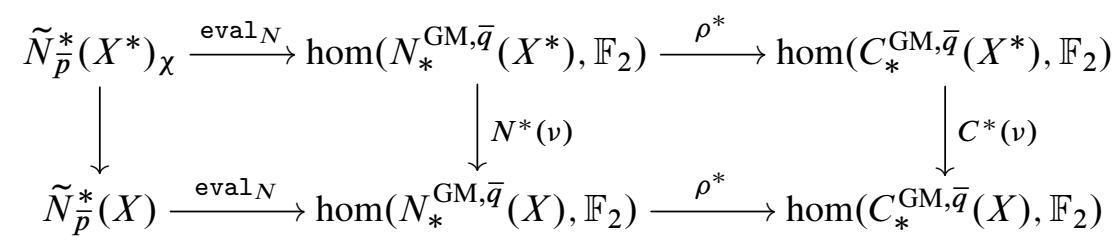

The right-hand square is commutative by naturality of $\rho_{*}$. We prove now the commutativity of the left-hand one. Let $\Phi \in \tilde{N}_{\bar{p}}^{*}\left(X^{*}\right)$, and let $\sigma: \Delta_{\sigma}=\Delta^{j_{0}} * \cdots * \Delta^{j_{n}} \rightarrow X$ be a filtered simplex, with associated filtered simplex $\nu \circ \sigma: \Delta_{\nu \circ \sigma} \rightarrow X^{*}$. We have to check

$$
\left(N^{*}(\nu) \circ \operatorname{eval}_{N}(\Phi)\right)(\sigma)=\operatorname{eval}_{N}(\chi(\Phi))(\sigma)
$$

For a given $\sigma$, we can decompose $v$ in a finite number of elementary amalgamations and thus replace $\chi_{\sigma}: \tilde{N}^{*}\left(\Delta_{\nu \circ \sigma}\right) \rightarrow \tilde{N}^{*}\left(\Delta_{\sigma}\right)$ by $\xi_{i}: \tilde{N}^{*}(\Delta(i)) \rightarrow \tilde{N}^{*}(\Delta)$, as defined in (7) and (8). Set $\Phi_{\nu \circ \sigma}=\sum_{j} \Phi_{0, j} \otimes \cdots \otimes \Phi_{n, j} \in \tilde{N}^{*}(\Delta(i))$ and suppose $i \neq n-1$. By definition (7), we have

$$
\begin{aligned}
\chi(\Phi)_{\sigma} & =\chi_{\sigma}\left(\Phi_{\nu \circ \sigma}\right)=\xi_{i}\left(\Phi_{\nu \circ \sigma}\right) \\
& =\sum_{j} \Phi_{i, j}([\mathrm{v}]) \cdot \Phi_{0, j} \otimes \cdots \otimes \Phi_{i-1, j} \otimes \alpha\left(\Phi_{i+1, j}\right) \otimes \Phi_{i+2, j} \otimes \cdots \otimes \Phi_{n, j},
\end{aligned}
$$

and the right-hand side of (10) is equal to

$$
\begin{aligned}
\operatorname{eval}_{N}(\chi(\Phi))(\sigma)=\sum_{j}\left(\Phi_{0, j}\left(\left[c \Delta^{j_{0}}\right]\right) \cdots \Phi_{i-1, j}\left(\left[c \Delta^{j_{i}}\right]\right) \cdot \Phi_{i, j}([\mathrm{v}])\right. \\
\left.\cdot \alpha\left(\Phi_{i+1, j}\right)\left(\left[c \Delta^{j_{i}}\right] \otimes\left[c \Delta^{j_{i+1}}\right]\right) \cdots \Phi_{n, j}\left(\left[\Delta^{j_{n}}\right]\right)\right) .
\end{aligned}
$$


We determine now the left-hand side of (10):

$$
\begin{aligned}
\left(N^{*}(v) \circ \operatorname{eval}_{N}(\Phi)\right)(\sigma) & =\operatorname{eval}_{N}(\Phi)(v \circ \sigma) \\
& =\sum_{j}\left(\Phi_{0, j}\left(\left[c \Delta^{j_{0}}\right]\right) \cdots \Phi_{i-1, j}\left(\left[c \Delta^{j_{i-1}}\right]\right) \cdot \Phi_{i, j}([\mathrm{v}])\right. \\
\cdot & \Phi_{i+1, j}\left(\left[c \Delta^{j_{i}+j_{i+1}+1}\right]\right) \cdots \Phi_{n, j}\left(\left[c \Delta\left(j_{n}\right]\right)\right) .
\end{aligned}
$$

Thus the left-hand and the right-hand sides coincide by definition of $\alpha$. A similar argument gives the result when $i=n-1$.

We have established above that the two horizontal lines of the commutative square (9) are quasi-isomorphisms. The right-hand vertical map is a quasi-isomorphism also; see [15]. Thus $\chi: \tilde{N}_{\bar{p}}^{*}\left(X^{*}\right) \rightarrow \tilde{N}_{\bar{p}}^{*}(X)$ is a quasi-isomorphism.

Proof of Lemma 7.2 We first consider the map $\alpha$ and its behavior with restriction maps. Let $\nabla_{a}$ and $\nabla_{b}$ be faces of $\Delta^{a}$ and $\Delta^{b}$, respectively, including the cases $\nabla_{a}=\varnothing$ or $\nabla_{b}=\varnothing$. Then the diagram

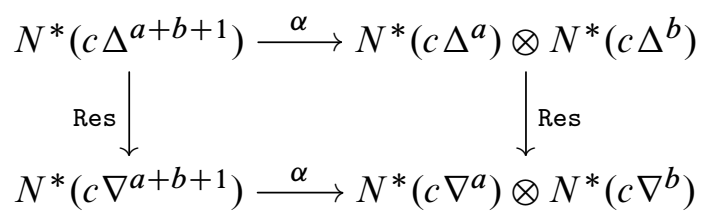

commutes, where the two vertical maps are given by the restriction map. To verify this assertion, we consider two faces, $F_{a}$ of $\nabla_{a}$ and $F_{b}$ of $\nabla_{b}$, and check the commutativity for the cochain $1_{c\left(F_{a} * F_{b}\right)}$, the other cases being similar:

$$
\begin{aligned}
& \operatorname{Res}\left(\alpha\left(1_{c\left(F_{a} * F_{b}\right)}\right)\right)=\operatorname{Res}\left(1_{c F_{a}} \otimes 1_{c F_{b}}\right)=1_{c F_{a}} \otimes 1_{c F_{b}} \\
& =\alpha\left(1_{c}\left(F_{a} * F_{b}\right)\right)=\alpha\left(\operatorname{Res}\left(1_{c}\left(F_{a} * F_{b}\right)\right)\right) .
\end{aligned}
$$

Now, comes the differential. Let $L$ be a finite simplicial complex endowed with a partial order of its vertices such that the vertices of any simplex are simply ordered. In the cone $c L$, the cone point is the greatest element. In the sequel, we adopt the following; see [22, page 292].

\section{Steenrod's convention}

A symbol, such as $F, G$ or $\nabla$, will denote, ambiguously, either (1) a simplex of $L$, or (2) the array of vertices of the simplex ordered as in $L$, or (3) the orientation of the simplex determined by this order, or (4) the elementary cochain which attaches +1 to this oriented simplex and 0 to all others. The ambiguity can usually be resolved by examining the context in which the symbol is used. 
With this convention, the definition of $\alpha: N^{*}\left(c \Delta^{a+b+1}\right) \rightarrow N^{*}\left(c \Delta^{a}\right) \otimes N^{*}\left(c \Delta^{b}\right)$ can be written as

$$
\begin{cases}\alpha\left(c\left(F_{a} * F_{b}\right)\right)=c F_{a} \otimes c F_{b} & \text { the cases } F_{a}=\varnothing \text { and } F_{b}=\varnothing \text { being included, } \\ \alpha\left(F_{a} * F_{b}\right)=c F_{a} \otimes F_{b} & \text { if } F_{b} \neq \varnothing, \text { the case } F_{a}=\varnothing \text { being included, } \\ \alpha\left(F_{a}\right)=F_{a} \otimes \mathrm{v}_{b}+F_{a} \otimes \mathbb{V}_{b}, & \end{cases}
$$

where $\mathbb{V}_{b}$ denotes the sum of the vertices of $\Delta^{b}$. The definition of the coboundary with this convention is also specified in [22, page 296]. Let $F_{a}=\left(a_{0}, \ldots, a_{k}\right)$ be a nonempty face of a simplex $\Delta^{a}$; we denote by $c F_{a}=\left(a_{0}, \ldots, a_{k}, \mathrm{v}_{a}\right)$ the face obtained from the adjunction of the cone point $\mathrm{v}_{a}$. It is important to observe that, in this setting, the differential of a face $F$ (viewed as a cochain) depends on the simplicial complex in which we do the computation. For instance, the differentials $\delta^{a}$ in $\Delta^{a}$ and $\delta^{c a}$ in $c \Delta^{a}$ are linked by

$$
\left\{\begin{aligned}
\delta^{c a}\left(c F_{a}\right) & =c\left(\delta^{a} F_{a}\right), \\
\delta^{c a} F_{a} & =\delta^{a} F_{a}+c F_{a} \\
\delta^{c a} \mathrm{v}_{a} & =c \mathbb{V}_{a}
\end{aligned}\right.
$$

where $\mathbb{V}_{a}$ is the sum of the vertices of $\Delta^{a}$. If $F_{b}$ is a nonempty face in $\Delta^{b}$, the differential $\delta^{a * b}$ in $\Delta^{a} * \Delta^{b}$ is defined by

$$
\left\{\begin{aligned}
\delta^{a * b}\left(F_{a} * F_{b}\right) & =\left(\delta^{a} F_{a}\right) * F_{b}+F_{a} *\left(\delta^{b} F_{b}\right), \\
\delta^{a * b} F_{a} & =\delta^{a} F_{a}+F_{a} * \mathbb{V}_{b} \\
\delta^{a * b} F_{b} & =\mathbb{V}_{a} * F_{b}+\delta^{b} F_{b} .
\end{aligned}\right.
$$

The differential on $c\left(\Delta^{a} * \Delta^{b}\right)=\left(c \Delta^{a}\right) * \Delta^{b}$ can be deduced from the combination of the previous equalities; we denote it by $\delta^{c(a * b)}$. We make the notations uniform by setting $\delta^{c a \otimes c b}$ and $\delta^{c a \otimes b}$ to the product differentials on $N^{*}\left(c \Delta^{a}\right) \otimes N^{*}\left(c \Delta^{b}\right)$ and $N^{*}\left(c \Delta^{a}\right) \otimes N^{*}\left(\Delta^{b}\right)$, respectively. We now verify the compatibility of $\alpha$ with the differentials by considering the various cases:

- Suppose $F_{a} \neq \varnothing$ and $F_{b} \neq \varnothing$ :

$$
\begin{aligned}
\left(\delta^{c a \otimes c b}\right)\left(\alpha\left(c\left(F_{a} * F_{b}\right)\right)\right) & =\left(\delta^{c a \otimes c b}\right)\left(c F_{a} \otimes c F_{b}\right) \\
& =\delta^{c a}\left(c F_{a}\right) \otimes c F_{b}+c F_{a} \otimes \delta^{c b}\left(c F_{b}\right) \\
& =c \delta^{a} F_{a} \otimes c F_{b}+c F_{a} \otimes c \delta^{b} F_{b} \\
& =\alpha\left(c\left(\left(\delta^{a} F_{a}\right) * F_{b}\right)\right)+\alpha\left(c\left(F_{a} *\left(\delta^{b} F_{b}\right)\right)\right) \\
& =\alpha\left(c\left(\delta^{a * b}\left(F_{a} * F_{b}\right)\right)\right) \\
& =\alpha\left(\delta^{c(a * b)} c\left(F_{a} * F_{b}\right)\right),
\end{aligned}
$$




$$
\begin{aligned}
\left(\delta^{c a \otimes c b}\right) \alpha\left(F_{a} * F_{b}\right) & =\left(\delta^{c a \otimes c b}\right)\left(c F_{a} \otimes F_{b}\right) \\
& =\left(\delta^{c a} c F_{a}\right) \otimes F_{b}+c F_{a} \otimes\left(\delta^{c b} F_{b}\right) \\
& =\left(c \delta^{a} F_{a}\right) \otimes F_{b}+c F_{a} \otimes\left(\delta^{b} F_{b}\right)+c F_{a} \otimes c F_{b} \\
& =\alpha\left(\left(\delta^{a} F_{a}\right) * F_{b}\right)+\alpha\left(F_{a} *\left(\delta^{b} F_{b}\right)\right)+\alpha\left(c\left(F_{a} * F_{b}\right)\right) \\
& =\alpha\left(\delta^{a * b}\left(F_{a} * F_{b}\right)+c\left(F_{a} * F_{b}\right)\right) \\
& =\alpha\left(\delta^{c(a * b)}\left(F_{a} * F_{b}\right)\right) .
\end{aligned}
$$

- Suppose $F_{a} \neq \varnothing$ and $F_{b}=\varnothing$ :

$$
\begin{aligned}
\left(\delta^{c a \otimes c b}\right) \alpha\left(c F_{a}\right) & =\left(\delta^{c a \otimes c b}\right)\left(c F_{a} \otimes \mathrm{v}_{b}\right)=\left(\delta^{c a} c F_{a}\right) \otimes \mathrm{v}_{b}+c F_{a} \otimes \delta^{c b} \mathrm{v}_{b} \\
& =\left(c \delta^{a} F_{a}\right) \otimes \mathrm{v}_{b}+\left(c F_{a}\right) \otimes c \mathbb{V}_{b}=\alpha\left(c\left(\delta^{a} F_{a}+F_{a} * \mathbb{V}_{b}\right)\right) \\
& =\alpha\left(c\left(\delta^{a * b} F_{a}\right)\right)=\alpha\left(\delta^{c(a * b)} c F_{a}\right), \\
\left(\delta^{c a \otimes c b}\right) \alpha\left(F_{a}\right) & =\left(\delta^{c a \otimes c b}\right)\left(F_{a} \otimes \mathrm{v}_{b}+F_{a} \otimes \mathbb{V}_{b}\right) \\
& =\delta^{c a} F_{a} \otimes\left(\mathrm{v}_{b}+\mathbb{V}_{b}\right)+F_{a} \otimes \delta^{c b}\left(\mathrm{v}_{b}+\mathbb{V}_{b}\right) \\
& =\left(\delta^{a} F_{a}\right) \otimes\left(\mathrm{v}_{b}+\mathbb{V}_{b}\right)+c F_{a} \otimes \mathrm{v}_{b}+c F_{a} \otimes \mathbb{V}_{b}+0 \\
& =\alpha\left(\delta^{a} F_{a}\right)+\alpha\left(c F_{a}\right)+\alpha\left(F_{a} * \mathbb{V}_{b}\right) \\
& =\alpha\left(\delta^{c a} F_{a}+c F_{a}\right) \\
& =\alpha\left(\delta^{c(a * b)} F_{a}\right) .
\end{aligned}
$$

(We have used $\delta^{c a} F_{a}=\delta^{a} F_{a}+c F_{a}$ and $\delta^{c b}\left(\mathrm{v}_{b}+\mathbb{V}_{b}\right)=0$. For the last one, observe that $\mathrm{v}_{b}+\mathbb{V}_{b}$ is the nontrivial cocycle in degree 0 .)

- Suppose $F_{a}=\varnothing$ and $F_{b} \neq \varnothing$ :

$$
\begin{aligned}
\left(\delta^{c a \otimes c b}\right) \alpha\left(c F_{b}\right) & =\left(\delta^{c a \otimes c b}\right)\left(\mathrm{v}_{a} \otimes c F_{b}\right)=c \mathbb{V}_{a} \otimes c F_{b}+\mathrm{v}_{a} \otimes c \delta^{b} F_{b} \\
& =\alpha\left(c\left(\mathbb{V}_{a} * F_{b}\right)\right)+\alpha\left(c \delta^{b} F_{b}\right)=\alpha\left(c \delta^{a * b} F_{b}\right) \\
& =\alpha\left(\delta^{c(a * b)} c F_{b}\right), \\
\left(\delta^{c a \otimes c b}\right) \alpha\left(F_{b}\right) & =\left(\delta^{c a \otimes c b}\right)\left(\mathrm{v}_{a} \otimes F_{b}\right)=c \mathbb{V}_{a} \otimes F_{b}+\mathrm{v}_{a} \otimes \delta^{c b} F_{b} \\
& =c \mathbb{V}_{a} \otimes F_{b}+\mathrm{v}_{a} \otimes \delta^{b} F_{b}+\mathrm{v}_{a} \otimes c F_{b} \\
& =\alpha\left(\mathbb{V}_{a} * F_{b}\right)+\alpha\left(\delta^{b} F_{b}\right)+\alpha\left(c F_{b}\right) \\
& =\alpha\left(\delta^{c(a * b)} F_{b}\right) .
\end{aligned}
$$


- Suppose $F_{a}=F_{b}=\varnothing$ :

$$
\begin{aligned}
\left(\delta^{c a \otimes c b}\right) \alpha(c \varnothing) & =\left(\delta^{c a \otimes c b}\right)\left(\mathrm{v}_{a} \otimes \mathrm{v}_{b}\right)=c \mathbb{V}_{a} \otimes \mathrm{v}_{b}+\mathrm{v}_{a} \otimes c \mathbb{V}_{b} \\
& =\alpha\left(c \mathbb{V}_{a}+c \mathbb{V}_{b}\right)=\alpha\left(c \mathbb{V}_{a+b+1}\right) \\
& =\alpha\left(\delta^{c(a * b)} c \varnothing\right) .
\end{aligned}
$$

As for the map $\beta: N^{*}\left(\Delta^{a+b+1}\right) \rightarrow N^{*}\left(c \Delta^{a}\right) \otimes N^{*}\left(\Delta^{b}\right)$, its description with Steenrod's convention is written:

$$
\left\{\begin{array}{l}
\beta\left(F_{a} * F_{b}\right)=c F_{a} \otimes F_{b} \\
\beta\left(F_{a}\right)=F_{a} \otimes \mathbb{V}_{b} .
\end{array}\right.
$$

The proof of its compatibility with restriction maps is totally similar to the proof done for $\alpha$. Therefore, we are reduced to check the compatibility of $\beta$ with the differentials. As before, we list the different cases:

- Suppose $F_{a} \neq \varnothing$ and $F_{b} \neq \varnothing$ :

$$
\begin{aligned}
\left(\delta^{c a \otimes b}\right)\left(\beta\left(F_{a} * F_{b}\right)\right) & =\left(\delta^{c a \otimes b}\right)\left(c F_{a} \otimes F_{b}\right)=\left(\delta^{c a} c F_{a}\right) \otimes F_{b}+c F_{a} \otimes\left(\delta^{b} F_{b}\right) \\
& =\left(c \delta^{a} F_{a}\right) \otimes F_{b}+c F_{a} \otimes\left(\delta^{b} F_{b}\right) \\
& =\beta\left(\left(\delta^{a} F_{a}\right) * F_{b}\right)+\beta\left(F_{a} *\left(\delta^{b} F_{b}\right)\right) \\
& =\beta\left(\delta^{a * b}\left(F_{a} * F_{b}\right)\right) .
\end{aligned}
$$

- Suppose $F_{a} \neq \varnothing$ and $F_{b}=\varnothing$ :

$$
\begin{aligned}
\left(\delta^{c a \otimes b}\right)\left(\beta\left(F_{a}\right)\right) & =\left(\delta^{c a \otimes b}\right)\left(F_{a} \otimes \mathbb{V}_{b}\right)=\left(\delta^{c a} F_{a}\right) \otimes \mathbb{V}_{b}+F_{a} \otimes\left(\delta^{b} \mathbb{V}_{b}\right) \\
& =\left(\delta^{a} F_{a}\right) \otimes \mathbb{V}_{b}+\left(c F_{a}\right) \otimes \mathbb{V}_{b}+0=\beta\left(\delta^{a} F_{a}+F_{a} * \mathbb{V}_{b}\right) \\
& =\beta\left(\delta^{a * b} F_{a}\right) .
\end{aligned}
$$

- Suppose $F_{a}=\varnothing$ and $F_{b} \neq \varnothing$ :

$$
\begin{aligned}
\left(\delta^{c a \otimes b}\right)\left(\beta\left(F_{b}\right)\right) & =\left(\delta^{c a \otimes b}\right)\left(\mathrm{v}_{a} \otimes F_{b}\right)=\left(c \mathbb{V}_{a}\right) \otimes F_{b}+\mathrm{v}_{a} \otimes\left(\delta^{b} F_{b}\right) \\
& =\beta\left(\mathbb{V}_{a} * F_{b}\right)+\beta\left(\delta^{b} F_{b}\right)=\beta\left(\delta^{a * b} F_{b}\right) .
\end{aligned}
$$

Proof of Lemma 7.3 The compatibilities with restriction maps and differentials being local, they are direct consequences of Lemma 7.2. We now study the behavior of $\xi_{i}$ with the perverse degrees.

We continue with Steenrod's convention and begin with the expression of the perverse degree in this context. Let $F=F_{0} \otimes \cdots \otimes F_{n}$ be a tensor product of nonempty faces in $\widetilde{\Delta}=c \Delta^{j_{0}} \times \cdots \times c \Delta^{j_{n-1}} \times \Delta^{j_{n}}$. In Steenrod's convention, we do not distinguish between $F$ and the tensor product of cochains, $1_{F_{0}} \otimes \cdots \otimes 1_{F_{n}}$. We observe that, if a 
face $F_{k}$ of $c \Delta^{j_{k}}$ is not included in $\Delta^{j_{k}}$, then the cochain $1_{F_{k}}$ restricts to 0 on the subcomplex $\Delta^{j_{k}} \times\{1\}$ of $c \Delta^{j_{k}}$. Therefore, by Definition 1.2, the perverse degree of $F$ is given by

$$
\left\|F_{0} \otimes \cdots \otimes F_{n}\right\|_{\ell}= \begin{cases}-\infty & \text { if } F_{n-\ell} \not \subset \Delta^{j_{n-\ell}}, \\ \left|F_{n-\ell+1}\right|+\cdots+\left|F_{n}\right| & \text { if } F_{n-\ell} \subset \Delta^{j_{n-\ell}},\end{cases}
$$

for any $\ell \in\{1, \ldots, n\}$. A similar definition occurs for the blow-up $\widetilde{\Delta(i)}$ of $\Delta(i)$.

As $\xi_{i}$ is compatible with the differentials, it is sufficient to prove that the image of a $\bar{p}$-admissible cochain is $\bar{p}$-admissible. Let $\nabla=\nabla_{0} \otimes \cdots \otimes \nabla_{n}$ be a tensor product of faces of $\widetilde{\Delta(i)}$ such that

$$
\left\|\nabla_{0} \otimes \cdots \otimes \nabla_{n}\right\|_{\ell} \leq \bar{p}(\ell) \text { for any } \ell \in\{1, \ldots, n\} .
$$

As we are dealing with $\Delta(i)$ (see (6)), we have $\nabla_{i}=c \varnothing$ and, with the notations of (7), $\Phi_{i j}([\mathrm{v}])=1$. Thus,

$\xi_{i}\left(\nabla_{0} \otimes \cdots \otimes \nabla_{n}\right)= \begin{cases}\nabla_{0} \otimes \cdots \otimes \nabla_{i-1} \otimes \alpha\left(\nabla_{i+1}\right) \otimes \nabla_{i+2} \otimes \cdots \otimes \nabla_{n}, & \text { if } i \neq n-1, \\ \nabla_{0} \otimes \cdots \otimes \nabla_{n-2} \otimes \beta\left(\nabla_{n}\right), & \text { if } i=n-1 .\end{cases}$

The morphisms $\alpha$ and $\beta$ preserving the dimension of faces, it is sufficient to consider the following cases:

- Suppose $i \neq n-1$ and $n-\ell=i$. Then $\ell \neq 1$, and we have $\left\|\xi_{i}\left(\nabla_{0} \otimes \cdots \otimes \nabla_{n}\right)\right\|_{\ell}$

$$
\begin{aligned}
& = \begin{cases}-\infty & \text { if } \nabla_{i+1}=\nabla_{a} * \nabla_{b} \text { with } \nabla_{b} \neq \varnothing, \\
\max (|\mathrm{v}|,|\mathbb{V}|)+\left|\nabla_{n-\ell+2}\right|+\cdots+\left|\nabla_{n}\right| & \text { or if } \nabla_{i+1}=c\left(\nabla_{a} * \nabla_{b}\right),\end{cases} \\
& \leq\left\|\nabla_{0} \otimes \cdots \otimes \nabla_{n}\right\|_{\ell-1} \leq \bar{p}(\ell-1) \leq \bar{p}(\ell) .
\end{aligned}
$$

We have used here that the perversity $\bar{p}$ is order-preserving and $|\mathrm{v}|=|\mathbb{V}|=0$ in $c \Delta^{j_{n-\ell+1}}$.

- Suppose $i \neq n-1$ and $n-\ell=i+1$. We have

$$
\begin{aligned}
\left\|\xi_{i}\left(\nabla_{0} \otimes \cdots \otimes \nabla_{n}\right)\right\|_{\ell} & = \begin{cases}-\infty & \text { if } \nabla_{i+1}=c\left(\nabla_{a} * \nabla_{b}\right), \\
\left|\nabla_{n-\ell+1}\right|+\cdots+\left|\nabla_{n}\right| & \text { if } \nabla_{i+1}=\nabla_{a} * \nabla_{b} \text { or }\end{cases} \\
& \leq\left\|\nabla_{0} \otimes \cdots \otimes \nabla_{n}\right\|_{\ell} \leq \bar{p}(\ell) .
\end{aligned}
$$

- Suppose $i=n-1$ and $\ell=1$. We have

$$
\begin{aligned}
\left\|\xi_{i}\left(\nabla_{0} \otimes \cdots \otimes \nabla_{n}\right)\right\|_{1} & = \begin{cases}-\infty & \text { if } \nabla_{n}=\nabla_{a} * \nabla_{b} \text { with } \nabla_{b} \neq \varnothing \\
|\mathbb{V}| & \text { if } \nabla_{n}=\nabla_{a}\end{cases} \\
& \leq 0=\bar{p}(1) .
\end{aligned}
$$




\section{Steenrod's definition of cup $_{i}$-products}

Let $L$ be a finite simplicial complex endowed with a partial order of its vertices such that the vertices of any simplex are simply ordered. Let $F=\left(a_{0}, \ldots, a_{k}\right)$ and $G=\left(b_{0}, \ldots, b_{\ell}\right)$ be two (ordered) simplices of $L$, and let $i \geq 0$ be an integer. The ordered pair $(F, G)$ is called $i$-regular if $F$ and $G$ have exactly $(i+1)$ vertices in common, $\left(c_{0}, \ldots, c_{i}\right)$, such that

- $c_{0}=b_{0}$,

- $\quad c_{j}$ and $c_{j+1}$ are adjacent in $F$ if $j$ is even and adjacent in $G$ if $j$ is odd, for all $0 \leq j<i$,

- $\quad c_{i}$ is the last vertex of $F$ if $i$ is even and the last vertex of $G$ if $i$ is odd.

Denote by $F_{0}$ the face of $F$ spanned by its vertices lower than or equal to $c_{0}$ and by $F_{2 j}$ the face of $F$ spanned by its vertices greater than or equal to $c_{2 j-1}$ and lower than or equal to $c_{2 j},(0<2 j \leq i)$. If $i$ is odd, let $F_{i+1}$ be the face of $F$ spanned by its vertices greater than or equal to $c_{i}$. We do a similar decomposition for $G$, denoting by $G_{2 j+1}(1 \leq 2 j+1<i+1)$ the face of $G$ spanned by its vertices greater than or equal to $c_{2 j}$ and lower than or equal to $c_{2 j+1}$. If $i$ is even, let $G_{i+1}$ be the face spanned by the vertices greater than or equal to $c_{i}$. This gives the decompositions

$$
F=F_{0} * F_{2} * \cdots * F_{2 s} \text { and } G=G_{1} * G_{3} * \cdots * G_{2 s+(-1)^{i}},
$$

with $2 s=i$ if $i$ is even and $2 s=i+1$ if $i$ is odd.

Now, we denote by $G_{2 j+1}^{\prime}$ the face of $G_{2 j+1}$ obtained by deleting the vertices $c_{2 j}$ and $c_{2 j+1}$. Moreover, if $i$ is even, let $G_{i+1}^{\prime}$ be the face of $G_{i+1}$ obtained by deleting $c_{i}$.

Definition 7.4 We define $F \cup_{i} G=0$ in the group of $(k+\ell-i)$-cochains, if the couple $(F, G)$ is not $i$-regular, and otherwise, by

$$
F \cup_{i} G=F_{0} * G_{1}^{\prime} * F_{2} * G_{3}^{\prime} * \cdots * \begin{cases}G_{i+1}^{\prime} & \text { if } i \text { is even, } \\ F_{i+1} & \text { if } i \text { is odd }\end{cases}
$$

Example 7.5 We give an illustration of the cases $i$ even and $i$ odd in low dimensions.

(1) The pair $\left(F=\left(a_{0}, \ldots, a_{k}\right), G=\left(b_{0}, \ldots, b_{\ell}\right)\right)$ is 0 -regular if $a_{k}=b_{0}$. We write their vertices as follows:

$$
\begin{array}{ll}
F: & a_{0}---a_{k} \\
& \|: \\
& b_{0}---b_{\ell}
\end{array}
$$


By definition, $F \cup_{0} G=\left(a_{0}, \ldots, a_{k}, b_{1}, \ldots, b_{\ell}\right)$ is the (classical) cup-product.

(2) The pair $(F, G)$ is 1 -regular if they have two common vertices $\left(c_{0}, c_{1}\right)$ such that the vertices of $F$ and $G$ can be put in two lines, as follows:

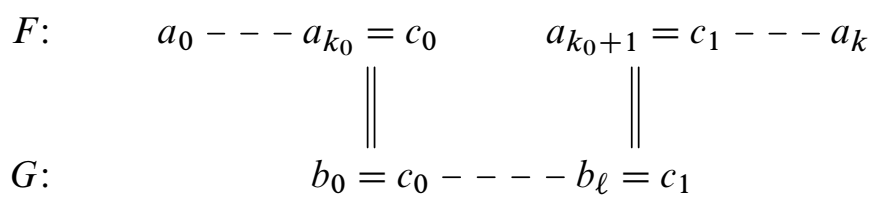

By definition, $F \cup_{1} G=\left(a_{0}, \ldots, a_{k_{0}}, b_{1}, \ldots, b_{\ell-1}, a_{k_{0}+1}, \ldots, a_{k}\right)$.

(3) The pair $(F, G)$ is 2 -regular if they have three common vertices $\left(c_{0}, c_{1}, c_{2}\right)$ such that the vertices of $F$ and $G$ can be put in two lines as:

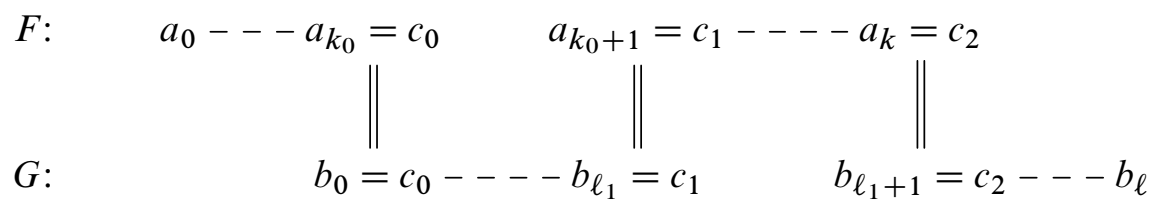

By definition, $F \cup_{2} G=\left(a_{0}, \ldots, a_{k_{0}}, b_{1}, \ldots, b_{\ell_{1}-1}, a_{k_{0}+1}, \ldots, a_{k}, b_{\ell_{1}+2}, \ldots, b_{\ell}\right)$.

For the convenience of the reader, we recall the next statement of Steenrod, written with the notation of this paper.

Proposition 7.6 [22, Theorem, page 295] The Steenrod squares satisfy:

(1) $F \cup_{i}(c G)= \begin{cases}c\left(F \cup_{i} G\right) & \text { if } i \text { is even, } \\ 0 & \text { if } i \text { is odd, }\end{cases}$

(2) $(c F) \cup_{i} G= \begin{cases}0 & \text { if } i \text { is even, } \\ c\left(F \cup_{i} G\right) & \text { if } i \text { is odd, }\end{cases}$

(3) $(c F) \cup_{i}(c G)=c\left(F \cup_{i-1} G\right)$.

The domains of the functions $\alpha$ and $\beta$ are the euclidean simplices $\Delta^{a+b+1}=\Delta^{a} * \Delta^{b}$ and $c\left(\Delta^{a} * \Delta^{b}\right)$. Therefore, we need to study the $\operatorname{cup}_{i}$-products in these complexes. The case of the cone $c\left(\Delta^{a} * \Delta^{b}\right)$ can be deduced from the first one, $\Delta^{a+b+1}$, with Proposition 7.6. We order the vertices of $\Delta^{a+b+1}$ such that any vertex of $\Delta^{a}$ is lower than any vertex of $\Delta^{b}$. Also, the cone point $\mathrm{v}$ is the greatest element of the set of vertices. Recall from (3) the notation

$$
F \cup_{i}^{j} G= \begin{cases}F \cup_{i} G & \text { if } j \text { is even } \\ G \cup_{i} F & \text { if } j \text { is odd. }\end{cases}
$$


Lemma 7.7 Let $F_{a}$ and $G_{a}$ be (nonempty) faces of $\Delta^{a}$, and let $F_{b}$ and $G_{b}$ be (nonempty) faces of $\Delta^{b}$. For any $i>0$, we have, in $\Delta^{a} * \Delta^{b}$,

$$
\left(F_{a} * F_{b}\right) \cup_{i}\left(G_{a} * G_{b}\right)=\sum_{i_{1}+i_{2}=i-1}\left(F_{a} \cup_{i_{1}} G_{a}\right) *\left(F_{b} \cup_{i_{2}}^{i_{1}+1} G_{b}\right) .
$$

Note that the right-hand side of the equality (11) has at most one nonzero term.

If we set $-\cup_{-1}-=0$, the right hand side of (11) is equal to zero in the case $i=0$. Note also that $F_{a}$ and $G_{b}$ cannot have a common vertex, and neither can $F_{b}$ and $G_{a}$. Therefore, with the hypotheses of Lemma 7.7, the simplices $F_{a} * F_{b}$ and $G_{a} * G_{b}$ cannot have exactly one vertex in common and respect the convention on the order of the vertices. As a consequence, the equality (11) is also true for $i=0$, with the two sides equal to zero.

Proof The $\operatorname{cup}_{i}$-product of $F=F_{a} * F_{b}$ and $G=G_{a} * G_{b}$ is not zero only if $F$ and $G$ have $(i+1)$ vertices in common. Denote by $(x+1)$, with $0 \leq x \leq i$, the number of vertices in common for $F_{a}$ and $G_{a}$. Thus $F_{b}$ and $G_{b}$ have $(i-x)$ vertices in common. We also observe that the only nonzero term of the right-hand side of (11) corresponds to $i_{1}=x$.

Suppose $i$ is even and $x$ is odd. With the previous notation, we decompose

$$
\begin{aligned}
F_{a}=F_{a, 0} * \cdots * F_{a, x+1} & \text { and } \quad G_{a}=G_{a, 1} * \cdots * G_{a, x}, \\
F_{b}=F_{b, 0} * \cdots * F_{b, i-x-1} & \text { and } \quad G_{b}=G_{b, 1} * \cdots * G_{b, i-x} .
\end{aligned}
$$

Thus we have

$$
\begin{aligned}
\left(F_{a} * F_{b}\right) \cup_{i}\left(G_{a} * G_{b}\right) & =F_{a, 0} * G_{a, 1}^{\prime} * \cdots * G_{a, x}^{\prime} * F_{a, x+1} * F_{b, 0} * G_{b, 1}^{\prime} * \cdots * G_{b, i-x}^{\prime} \\
& =\left(F_{a} \cup_{x} G_{a}\right) *\left(F_{b} \cup_{i-x-1} G_{b}\right) \\
& =\left(F_{a} \cup_{x} G_{a}\right) *\left(F_{b} \cup_{i-x-1}^{x+1} G_{b}\right) .
\end{aligned}
$$

Now suppose $i$ is even and $x$ is even. We decompose

$$
F_{a}=F_{a, 0} * \cdots * F_{a, x} \text { and } G_{a}=G_{a, 1} * \cdots * G_{a, x+1} .
$$

Thus we have

$$
F_{a} \cup_{x} G_{a}=F_{a, 0} * G_{a, 1}^{\prime} * \cdots * F_{a, x} * G_{a, x+1}^{\prime} .
$$

Note that $F_{a} \cup_{x} G_{a}$ contains all the vertices of $F \cup_{i} G$ belonging to $\Delta^{a}$. The first vertex in common between $F_{a}$ and $G_{a}$ is the first vertex of $G_{a}$. The number of common points in $\Delta^{a}$ being the odd number $x+1$, the last vertex of $\Delta^{a}$ in common must be the last vertex of $F_{a}$. Therefore, the first vertex of $\Delta^{b}$ in common is the first vertex of $F_{b}$. (See Example 7.8 for an illustration of this argument.) Thus, for writing this 
final part of vertices in $F \cup_{i} G$ as a $\operatorname{cup}_{i-x-1}$-product, we have to decompose $F_{b}$ and $G_{b}$ as

$$
G_{b}=G_{b, 0} * \cdots * G_{b, i-x} \quad \text { and } \quad F_{b}=F_{b, 1} * \cdots * F_{b, i-x-1} .
$$

We deduce

$$
\begin{aligned}
\left(F_{a} * F_{b}\right) \cup_{i}\left(G_{a} * G_{b}\right) & =F_{a, 0} * G_{a, 1}^{\prime} * \cdots * G_{a, x+1}^{\prime} * G_{b, 0} * F_{b, 1}^{\prime} * \cdots * F_{b, i-x-1}^{\prime} * G_{b, i-x} \\
& =\left(F_{a} \cup_{x} G_{a}\right) *\left(G_{b} \cup_{i-x-1} F_{b}\right) \\
& =\left(F_{a} \cup_{x} G_{a}\right) *\left(F_{b} \cup_{i-x-1}^{x+1} G_{b}\right) .
\end{aligned}
$$

In the case where $i$ is odd, the conclusion is obtained with totally similar arguments.

Example 7.8 We specialize, with $x=2$, the argument from the previous proof. Let

$$
F_{a} * F_{b}=\left(f_{0}^{a}, \ldots, f_{\ell}^{a}\right) *\left(f_{0}^{b}, \ldots, f_{k}^{b}\right)
$$

and

$$
G_{a} * G_{b}=\left(g_{0}^{a}, \ldots, g_{u}^{a}\right) *\left(g_{0}^{b}, \ldots, g_{v}^{b}\right) .
$$

The following diagram represents

$$
\begin{aligned}
& \left(F_{a} * F_{b}\right) \cup_{i}\left(G_{a} * G_{b}\right)=\left(F_{a} \cup_{2} G_{a}\right) *\left(F_{b} \cup_{i-3}^{3} G_{b}\right)=\left(F_{a} \cup_{2} G_{a}\right) *\left(G_{b} \cup_{i-3} F_{b}\right): \\
& F_{a} * F_{b}: \quad f_{0}^{a}--\left\|_{---}\right\|\left\|_{---g_{u}^{a} g_{0}^{b}}\right\|_{----}^{a} \| f_{---}^{b}
\end{aligned}
$$

Proposition 7.9 Let $X$ be an $n$-dimensional PL-pseudomanifold, and let $\bar{p}$ and $\bar{q}$ be GM-perversities. Then the quasi-isomorphism $\chi: \tilde{N}_{\bullet}^{*}\left(X^{*}\right) \rightarrow \tilde{N}_{\bullet}^{*}(X)$ induced by

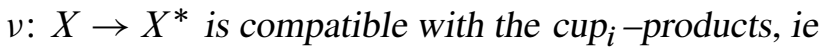

$$
\chi\left(\Phi \cup_{i} \Psi\right)=\chi(\Phi) \cup_{i} \chi(\Psi),
$$

for any $i \geq 0, \Phi \in \tilde{N}_{\bar{p}}^{r}\left(X^{*}\right), \Psi \in \tilde{N}_{\bar{q}}^{s}\left(X^{*}\right)$ and $\Phi \cup_{i} \Psi \in \tilde{N}_{\bar{p} \oplus \bar{q}}^{r+s}\left(X^{*}\right)$.

As $\operatorname{cup}_{i}$-products on $\tilde{N}^{*}(-)$ are defined locally, it is sufficient to do the proof for an elementary amalgamation. Thus Proposition 7.9 is a direct consequence of the next lemma.

Lemma 7.10 The two morphisms $\alpha: N^{*}\left(c \Delta^{a+b+1}\right) \rightarrow N^{*}\left(c \Delta^{a}\right) \otimes N^{*}\left(c \Delta^{b}\right)$ and $\beta: N^{*}\left(\Delta^{a+b+1}\right) \rightarrow N^{*}\left(c \Delta^{a}\right) \otimes N^{*}\left(\Delta^{b}\right)$ are compatible with the cup -products. 
Proof Consider the faces $F=F_{a} * F_{b}$ and $G=G_{a} * G_{b}$ of $\Delta^{a+b+1}$.

- First suppose that $F_{a} \neq \varnothing, F_{b} \neq \varnothing, G_{a} \neq \varnothing$ and $G_{b} \neq \varnothing$, and begin with the map $\beta$. We have to prove

$$
\beta\left(\left(F_{a} * F_{b}\right) \cup_{i}\left(G_{a} * G_{b}\right)\right)=\beta\left(F_{a} * F_{b}\right) \cup_{i} \beta\left(G_{a} * G_{b}\right) .
$$

From Lemma 7.7 and the definition of $\beta$, we get

$$
\beta\left(\left(F_{a} * F_{b}\right) \cup_{i}\left(G_{a} * G_{b}\right)\right)=\sum_{i_{1}+i_{2}=i-1}\left(c\left(F_{a} \cup_{i_{1}} G_{a}\right) \otimes\left(F_{b} \cup_{i_{2}}^{i_{1}+1} G_{b}\right) .\right.
$$

On the other side, we have

$$
\begin{aligned}
\beta\left(F_{a} * F_{b}\right) \cup_{i} \beta\left(G_{a} * G_{b}\right) & ={ }_{(1)}\left(c F_{a} \otimes F_{b}\right) \cup_{i}\left(c G_{a} \otimes G_{b}\right) \\
& ={ }_{(2)} \sum_{k=0}^{i}\left(c F_{a} \cup_{k} c G_{a}\right) \otimes\left(F_{b} \cup_{i-k}^{k} G_{b}\right) \\
& =(3) \sum_{k=1}^{i} c\left(F_{a} \cup_{k-1} G_{a}\right) \otimes\left(F_{b} \cup_{i-k}^{k} G_{b}\right) \\
& =\sum_{i_{1}+i_{2}=i-1}\left(c\left(F_{a} \cup_{i_{1}} G_{a}\right)\right) \otimes\left(F_{b} \cup_{i_{2}}^{i_{1}+1} G_{b}\right),
\end{aligned}
$$

where $=_{(1)}$ is the definition of $\beta,={ }_{(2)}$ comes from the structure of an $\mathcal{E}(2)$-algebra on a tensor product of $\mathcal{E}(2)$-algebras, recalled in Section 2, and $=_{(3)}$ is [22, Formula (4.3)], recalled in Proposition 7.6.

- With the same restriction, $F_{a} \neq \varnothing, F_{b} \neq \varnothing, G_{a} \neq \varnothing$ and $G_{b} \neq \varnothing$, we now study the map $\alpha$. The arguments are coming from Lemma 7.7, from the structure of $\mathcal{E}(2)$-algebra on a tensor product and from Proposition 7.6, as before. In the sequel, we use them without an explicit recall. We have only to study the cases where one of the faces contains the cone point, the other cases being already verified when we considered the map $\beta$.

(i) Let $c\left(F_{a} * F_{b}\right)$ and $c\left(G_{a} * G_{b}\right)$ be faces of $c \Delta^{a+b+1}$. Then we have

$$
\begin{aligned}
\alpha\left(c\left(F_{a} * F_{b}\right) \cup_{i} c\left(G_{a} * G_{b}\right)\right) & =\alpha\left(c\left(\left(F_{a} * F_{b}\right) \cup_{i-1}\left(G_{a} * G_{b}\right)\right)\right) \\
& =\alpha\left(c\left(\sum_{i_{1}+i_{2}=i-2}\left(F_{a} \cup_{i_{1}} G_{a}\right) *\left(F_{b} \cup_{i_{2}}^{i_{1}+1} G_{b}\right)\right)\right) \\
& =\sum_{i_{1}+i_{2}=i-2} c\left(F_{a} \cup_{i_{1}} G_{a}\right) \otimes c\left(F_{b} \cup_{i_{2}}^{i_{1}+1} G_{b}\right),
\end{aligned}
$$


and

$$
\begin{aligned}
\alpha\left(c\left(F_{a} * F_{b}\right)\right) \cup_{i} \alpha\left(c\left(G_{a} * G_{b}\right)\right) & =\left(c F_{a} \otimes c F_{b}\right) \cup_{i}\left(c G_{a} \otimes c G_{b}\right) \\
& =\sum_{k=0}^{i}\left(c F_{a} \cup_{k} c G_{a}\right) \otimes\left(c F_{b} \cup_{i-k}^{k} c G_{b}\right) \\
& =\sum_{k=1}^{i-1} c\left(F_{a} \cup_{k-1} G_{a}\right) \otimes c\left(F_{b} \cup_{i-k-1}^{k} G_{b}\right) \\
& =\sum_{i_{1}+i_{2}=i-2} c\left(F_{a} \cup_{i_{1}} G_{a}\right) \otimes c\left(F_{b} \cup_{i_{2}}^{i_{1}+1} G_{b}\right) .
\end{aligned}
$$

The compatibility with $\operatorname{cup}_{i}$-products is proved for these faces.

(ii) Let $c\left(F_{a} * F_{b}\right)$ and $\left(G_{a} * G_{b}\right)$ be faces of $c \Delta^{a+b+1}$. We have to prove that

$$
\alpha\left(c\left(F_{a} * F_{b}\right) \cup_{i}\left(G_{a} * G_{b}\right)\right)=\alpha\left(c\left(F_{a} * F_{b}\right)\right) \cup_{i} \alpha\left(G_{a} * G_{b}\right) .
$$

First observe that $\alpha\left(c\left(F_{a} * F_{b}\right) \cup_{i}\left(G_{a} * G_{b}\right)\right)=0$ if $i$ is even; see Proposition 7.6. We now study the $\operatorname{cup}_{i}$-product of the images under $\alpha$ :

$$
\begin{aligned}
\alpha\left(c\left(F_{a} * F_{b}\right)\right) \cup_{i} \alpha\left(G_{a} * G_{b}\right) & =\left(c F_{a} \otimes c F_{b}\right) \cup_{i}\left(c G_{a} \otimes G_{b}\right) \\
& =\sum_{k=0}^{i}\left(c F_{a} \cup_{k} c G_{a}\right) \otimes\left(c F_{b} \cup_{i-k}^{k} G_{b}\right) .
\end{aligned}
$$

We study the last right-hand side term in the case where $i$ is even.

- If $k$ is even, then $\left(c F_{b} \cup{ }_{i-k}^{k} G_{b}\right)=c F_{b} \cup \cup_{i-k} G_{b}=0$ since $i-k$ is even.

- If $k$ is odd, then $\left(c F_{b} \cup{ }_{i-k}^{k} G_{b}\right)=G_{b} \cup_{i-k} c F_{b}=0$ since $i-k$ is odd.

Thus (13) is satisfied for $i$ even.

Suppose now that $i$ is odd. The left-hand side of (13) can be expanded as

$$
\begin{aligned}
\alpha\left(c\left(F_{a} * F_{b}\right) \cup_{i}\left(G_{a} * G_{b}\right)\right) & =\alpha\left(c\left(\left(F_{a} * F_{b}\right) \cup_{i}\left(G_{a} * G_{b}\right)\right)\right) \\
& =\alpha\left(c\left(\sum_{i_{1}+i_{2}=i-1}\left(F_{a} \cup_{i_{1}} G_{a}\right) *\left(F_{b} \cup_{i_{2}}^{i_{1}+1} G_{b}\right)\right)\right) \\
& =\sum_{i_{1}+i_{2}=i-1} c\left(F_{a} \cup_{i_{1}} G_{a}\right) \otimes c\left(F_{b} \cup_{i_{2}}^{i_{1}+1} G_{b}\right) .
\end{aligned}
$$

We now consider the expression of the right-hand side of (13) already obtained:

$$
\sum_{k=0}^{i}\left(c F_{a} \cup \cup_{k} c G_{a}\right) \otimes\left(c F_{b} \cup \cup_{i-k}^{k} G_{b}\right)
$$


- For $k$ even, $i-k$ is odd, so $\left(c F_{b} \cup_{i-k}^{k} G_{b}\right)=c F_{b} \cup_{i-k} G_{b}=c\left(F_{b} \cup_{i-k} G_{b}\right)$.

- For $k$ odd, $i-k$ is even, so $\left(c F_{b} \cup_{i-k}^{k} G_{b}\right)=G_{b} \cup_{i-k} c F_{b}=c\left(G_{b} \cup_{i-k} F_{b}\right)$.

In conclusion, we have proved that $c F_{b} \cup \cup_{i-k}^{k} G_{b}=c\left(F_{b} \cup{ }_{i-k}^{k} G_{b}\right)$ and

$$
\begin{aligned}
\alpha\left(c\left(F_{a} * F_{b}\right)\right) \cup_{i} \alpha\left(G_{a} * G_{b}\right) & =\sum_{k=1}^{i} c\left(F_{a} \cup_{k-1} G_{a}\right) \otimes c\left(F_{b} \cup_{i-k}^{k} G_{b}\right) \\
& =\sum_{i_{1}+i_{2}=i-1} c\left(F_{a} \cup_{i_{1}} G_{a}\right) \otimes c\left(F_{b} \cup_{i_{2}}^{i_{1}+1} G_{b}\right) .
\end{aligned}
$$

We have established the compatibility with cup $_{i}$-products in this case.

(iii) Let $\left(F_{a} * F_{b}\right)$ and $c\left(G_{a} * G_{b}\right)$ be faces of $c \Delta^{a+b+1}$. This situation is similar to the previous one.

- We now consider the case where at least one of the subsets, $F_{a}, F_{b}, G_{a}$ and $G_{b}$, is the empty set, and we begin with the map $\beta$. The verification follows the same routine as above, but we cannot apply Lemma 7.7 in this situation. Therefore, we prove the compatibility with a direct computation of the two sides of the equality (12). We list the different possibilities with the values of the left-hand side (LHS) and of the right-hand side (RHS). If $F_{a}=F_{b}=\varnothing$ or $G_{a}=G_{b}=\varnothing$, the expressions become trivial and we may focus on the cases below.

Before doing these verifications, we note that $\mathbb{V}_{a}$ is a chain of vertices and, if $F_{a} \subset \Delta^{a}$ is given, one (and only one) of theses vertices, say $a_{t}$, is the first vertex of $F_{a}$. This implies $\mathbb{V}_{a} \cup_{0} F_{a}=\left(a_{t}\right) \cup_{0} F_{a}=F_{a}$. Similarly, we have $F_{a} \cup_{0} \mathbb{V}_{a}=F_{a}$, and $\mathbb{V}_{a}$ acts as a neutral element for $-\cup_{0}-$. Also, as $\mathrm{v}_{a} \notin F_{a}$, we have $\mathrm{v}_{a} \cup_{0} F_{a}=F_{a} \cup_{0} \mathrm{v}_{a}=0$.

(1) $F_{a}=\varnothing, F_{b} \neq \varnothing, G_{a}=\varnothing, G_{b} \neq \varnothing$.

LHS $=\beta\left(F_{b} \cup_{i} G_{b}\right)=\mathrm{v}_{a} \otimes\left(F_{b} \cup_{i} G_{b}\right)$.

$\mathrm{RHS}=\left(\mathrm{v}_{a} \otimes F_{b}\right) \cup_{i}\left(\mathrm{v}_{a} \otimes G_{b}\right)=\left(\mathrm{v}_{a} \cup_{0} \mathrm{v}_{a}\right) \otimes\left(F_{b} \cup_{i}^{0} G_{b}\right)=\mathrm{v}_{a} \otimes\left(F_{b} \cup_{i} G_{b}\right)$.

(2) $F_{a} \neq \varnothing, F_{b}=\varnothing, G_{a} \neq \varnothing, G_{b}=\varnothing$.

$\mathrm{LHS}=\beta\left(F_{a} \cup_{i} G_{a}\right)=\left(F_{a} \cup_{i} G_{a}\right) \otimes \mathbb{V}_{b}$.

$\mathrm{RHS}=\left(F_{a} \otimes \mathbb{V}_{b}\right) \cup_{i}\left(G_{a} \otimes \mathbb{V}_{b}\right)=\left(F_{a} \cup_{i} G_{a}\right) \otimes\left(\mathbb{V}_{b} \cup_{0}^{i} \mathbb{V}_{b}\right)=\left(F_{a} \cup_{i} G_{a}\right) \otimes \mathbb{V}_{b}$.

(3) $F_{a} \neq \varnothing, F_{b}=\varnothing, G_{a}=\varnothing, G_{b} \neq \varnothing$.

LHS $=\beta\left(F_{a} \cup_{i} G_{b}\right)=0$.

$\mathrm{RHS}=\left(F_{a} \otimes \mathbb{V}_{b}\right) \cup_{i}\left(\mathrm{v}_{a} \otimes G_{b}\right)=0$,

because $F_{a} \cup_{k} \vee_{a}=0$ for any $k$. 
(4) $F_{a}=\varnothing, F_{b} \neq \varnothing, G_{a} \neq \varnothing, G_{b}=\varnothing$.

$$
\begin{aligned}
& \mathrm{LHS}=\beta\left(F_{b} \cup_{i} G_{a}\right)=0 . \\
& \mathrm{RHS}=\beta\left(F_{b}\right) \cup_{i} \beta\left(G_{a}\right)=\left(\mathrm{v}_{a} \otimes F_{b}\right) \cup_{i}\left(G_{a} \otimes \mathbb{V}_{b}\right)=0,
\end{aligned}
$$

because $\mathrm{v}_{a} \cup_{k} G_{a}=0$ for any $k$.

(5) $F_{a}=\varnothing, F_{b} \neq \varnothing, G_{a} \neq \varnothing, G_{b} \neq \varnothing$.

$$
\mathrm{LHS}=\beta\left(F_{b} \cup_{i}\left(G_{a} * G_{b}\right)\right)=0,
$$

because $F_{b} \cap G_{a}=\varnothing$ and $G_{a} \neq \varnothing$.

$$
\begin{aligned}
\mathrm{RHS}=\beta\left(F_{b}\right) \cup_{i} \beta\left(G_{a} * G_{b}\right) & =\left(\mathrm{v}_{a} \otimes F_{b}\right) \cup_{i}\left(c G_{a} \otimes G_{b}\right) \\
& =\left(\mathrm{v}_{a} \cup_{0} c G_{a}\right) \otimes\left(F_{b} \cup_{i} G_{b}\right)=0,
\end{aligned}
$$

because the cone point $\mathrm{v}_{a}$ is the greatest vertex.

(6) $F_{a} \neq \varnothing, F_{b}=\varnothing, G_{a} \neq \varnothing, G_{b} \neq \varnothing$.

$$
\begin{aligned}
\text { LHS } & =\beta\left(F_{a} \cup_{i}\left(G_{a} * G_{b}\right)\right) \\
& = \begin{cases}\beta\left(\left(F_{a} \cup_{i} G_{a}\right) * G_{b}\right)=c\left(F_{a} \cup_{i} G_{a}\right) \otimes G_{b} & \text { for } i \text { even, } \\
0 & \text { for } i \text { odd. }\end{cases} \\
\text { RHS } & =\beta\left(F_{a}\right) \cup_{i} \beta\left(G_{a} * G_{b}\right)=\left(F_{a} \otimes \mathbb{V}_{b}\right) \cup_{i}\left(c G_{a} \otimes G_{b}\right) \\
& =\left(F_{a} \cup_{i} c G_{a}\right) \otimes\left(\mathbb{V}_{b} \cup_{0}^{i} G_{b}\right)= \begin{cases}c\left(F_{a} \cup_{i} G_{a}\right) \otimes G_{b} & \text { for } i \text { even, } \\
0 & \text { for } i \text { odd. }\end{cases}
\end{aligned}
$$

The nullity when $i$ is odd comes from Proposition 7.6.

(7) $F_{a} \neq \varnothing, F_{b} \neq \varnothing, G_{a}=\varnothing, G_{b} \neq \varnothing$.

$$
\begin{aligned}
\text { LHS } & =\beta\left(\left(F_{a} * F_{b}\right) \cup_{i} G_{b}\right)=\beta\left(F_{a} *\left(F_{b} \cup_{i} G_{b}\right)\right)=c F_{a} \otimes\left(F_{b} \cup_{i} G_{b}\right) . \\
\text { RHS } & =\beta\left(F_{a} * F_{b}\right) \cup_{i} \beta\left(G_{b}\right)=\left(c F_{a} \otimes F_{b}\right) \cup_{i}\left(\mathrm{v}_{a} \otimes G_{b}\right) \\
& =\left(c F_{a} \cup_{0} \mathrm{v}_{a}\right) \otimes\left(F_{b} \cup_{i} G_{b}\right)=c F_{a} \otimes\left(F_{b} \cup_{i} G_{b}\right) .
\end{aligned}
$$

(8) $F_{a} \neq \varnothing, F_{b} \neq \varnothing, G_{a} \neq \varnothing, G_{b}=\varnothing$.

$$
\begin{aligned}
\text { LHS } & =\beta\left(\left(F_{a} * F_{b}\right) \cup \cup_{i} G_{a}\right) \\
& = \begin{cases}0 & \text { for } i \text { even, } \\
\beta\left(\left(F_{a} \cup_{i} G_{a}\right) * F_{b}\right)=c\left(F_{a} \cup_{i} G_{a}\right) \otimes F_{b} & \text { for } i \text { odd. }\end{cases} \\
\text { RHS } & =\beta\left(F_{a} * F_{b}\right) \cup_{i} \beta\left(G_{a}\right)=\left(c F_{a} \otimes F_{b}\right) \cup_{i}\left(G_{a} \otimes \mathbb{V}_{b}\right)
\end{aligned}
$$

with the argument already used in the case (6). 
- The end of the proof is concerned with the map $\alpha$ when at least one of the subsets, $F_{a}, F_{b}, G_{a}$ and $G_{b}$, is the empty set. Computations are similar to the previous ones.

\section{References}

[1] C Berger, B Fresse, Combinatorial operad actions on cochains, Math. Proc. Cambridge Philos. Soc. 137 (2004) 135-174 MR

[2] A Borel, N Spaltenstein, Sheaf theoretic intersection cohomology, from: "Intersection cohomology", Progr. Math. 50, Birkhäuser, Boston, MA (1984) 47-182 MR

[3] J-P Brasselet, G Hector, M Saralegi, Théorème de de Rham pour les variétés stratifiées, Ann. Global Anal. Geom. 9 (1991) 211-243 MR

[4] D Chataur, M Saralegi-Aranguren, D Tanré, Intersection cohomology: simplicial blow-up and rational homotopy, preprint (2012) arXiv To appear in Mem. Amer. Math. Soc.

[5] G Friedman, Intersection homology of stratified fibrations and neighborhoods, Adv. Math. 215 (2007) 24-65 MR

[6] G Friedman, On the chain-level intersection pairing for PL pseudomanifolds, Homology, Homotopy Appl. 11 (2009) 261-314 MR

[7] G Friedman, Singular intersection homology, book in progress (2016) Available at http://faculty.tcu.edu/gfriedman/index.html

[8] G Friedman, J E McClure, Cup and cap products in intersection (co)homology, Adv. Math. 240 (2013) 383-426 MR

[9] R Godement, Topologie algébrique et théorie des faisceaux, Actualités Sci. Ind. 1252, Hermann, Paris (1958) MR

[10] M Goresky, R MacPherson, Intersection homology theory, Topology 19 (1980) 135162 MR

[11] M Goresky, R MacPherson, Intersection homology, II, Invent. Math. 72 (1983) 77$129 \mathrm{MR}$

[12] M Goresky, W Pardon, Wu numbers of singular spaces, Topology 28 (1989) 325-367 MR

[13] R M Goresky, Intersection homology operations, Comment. Math. Helv. 59 (1984) 485-505 MR

[14] M Hovey, Intersection homological algebra, from: "New topological contexts for Galois theory and algebraic geometry", (A Baker, B Richter, editors), Geom. Topol. Monogr. 16 (2009) 133-150 MR

[15] H C King, Topological invariance of intersection homology without sheaves, Topology Appl. 20 (1985) 149-160 MR 
[16] F Kirwan, J Woolf, An introduction to intersection homology theory, 2nd edition, Chapman \& Hall/CRC, Boca Raton, FL (2006) MR

[17] S Mac Lane, Categories for the working mathematician, 2nd edition, Graduate Texts in Mathematics 5, Springer, New York (1998) MR

[18] J P May, A general algebraic approach to Steenrod operations, from: "The Steenrod algebra and its applications", (F P Peterson, editor), Lecture Notes in Mathematics 168, Springer, Berlin (1970) 153-231 MR

[19] J W Milnor, J D Stasheff, Characteristic classes, Annals of Mathematics Studies 76, Princeton Univ. Press (1974) MR

[20] G Pollini, Intersection differential forms, Rend. Sem. Mat. Univ. Padova 113 (2005) 71-97 MR

[21] J-P Serre, Homologie singulière des espaces fibrés: Applications, Ann. of Math. 54 (1951) 425-505 MR

[22] N E Steenrod, Products of cocycles and extensions of mappings, Ann. of Math. 48 (1947) 290-320 MR

LAFMA, Université de Picardie Jules Verne

33, Rue Saint Leu, Villeneuve d'Ascq, 80039 Amiens Cedex 1, France

Laboratoire de Mathématiques de Lens, EA 2462, Université d'Artois

SP18, rue Jean Souvraz, 62307 Lens Cedex, France

Département de Mathématiques, UMR 8524, Université de Lille 1

Villeneuve D'Ascq, 59655 Lille Cedex, France

David.Chataur@u-picardie.fr, martin.saraleguiaranguren@univ-artois.fr, daniel.tanre@univ-lille1.fr

Received: 12 April 2014 Revised: 5 January 2015 